\title{
FOLDING MODEL POTENTIALS FROM REALISTIC INTERACTIONS FOR HEAVY-ION SCATTERING
}

\author{
G.R. SATCHLER \\ Oak Ridge Natınal Laboratory*, Oak Ridge, Tennessee 37830, U.S.A. \\ and \\ W.G. LOVE \\ Dept. of Physics and Astronomy, Lnitersity of Georgia**, Athens, Georgla 30602, U.S.A. \\ Received April 1979
}

Contents:

1. Introduction 185

2. Heavy ion potentials 186

3. The model 188

3.1. Double-folded potentials 188

3.2. Single-folded potentials 189

3.3. The effective interaction 190

3.4. The nuclear densities 196

3.5. General features 200

4. Application to nucleon scatternng 203

5. Application to alpha scattering 206

5.1. The $\alpha+\alpha$ interaction 206

5.2. The scattering of $\alpha+{ }^{40} \mathrm{Ca} \quad 206$

6. Applications to heavy-ion scatterıng 207

6.1. Procedure 207

6.2. The imaginary potential 210

6.3. Previous results 211

6.4. Present results 211
7. Scattering of ${ }^{6,7} \mathrm{Li}$ and ${ }^{9} \mathrm{Be}$

7.1. Density distributions for Li nuclei 224

7.2. Results for ${ }^{6} \mathrm{Li}$ scattering 227

7.3. The case of ${ }^{6} \mathrm{Li}+{ }^{40} \mathrm{Ca} \quad 227$

7.4. Discussion 228

7.5. The ${ }^{9} \mathrm{Be}$ nucleus $\quad 229$

8. Inelastic scattering 233

8.1. Introduction 233

8.2. Applications 234

8.3. Discussion 238

9. Summary and discussion 241

Appendix A: Spin and isospin effects in folded potentials 243

Appendix B: Momentum-space representation 245

Appendix C: Density-dependent interactions $\quad 249$

References $\quad 250$

Single orders for this issue

PHYSICS REPORTS (Review Section of Physics Letters) 55, No. 3 (1979) 183-254.

Copies of this issue may be obtained at the price given below. All orders should be sent directly to the Publisher. Orders must be accompanied by check.

Single issue price Dfl. 28.50 , postage included.

* Research sponsored by the Division of Physical Research, U.S. Department of Energy under contract W-7405-eng-26 with the Union Carbide Corporation.

** Work supported in part by the National Science Foundation. 


\title{
FOLDING MODEL POTENTIALS FROM REALISTIC INTERACTIONS FOR HEAVY-ION SCATTERING
}

\author{
G.R. SATCHLER \\ Oak Ridge National Laboratory, Oak Ridge, Tennessee 37830, U.S.A. \\ and
}

W.G. LOVE

Dept. of Physics and Astronomy, University of Georgia, Athens, Georgia 30602, U.S.A

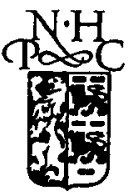

NORTH-HOLLAND PUBLISHING COMPANY - AMSTERDAM 
Abstract.

The double-folding model, with "realistıc" nucleon-nucleon interactions based upon a $G$-matrix constructed from the Reid potential, is used to calculate the real part of the optical potential for heavy-ion scattering. The resulting potentials are shown to reproduce the observed elastic scattering for a large number of systems with bombarding energies from 5 to $20 \mathrm{MeV}$ per nucleon. Some representative inelastic transitions are also reproduced. Exceptions are the elastic scattering of ${ }^{6} \mathrm{Li}$ and ${ }^{9} \mathrm{Be}$ for which the folded potentials must be reduced in strength by a factor of about two.

The same effective interactions are shown to give a good account of two particular cases of alpha scattering as well as some cases of nucleon-nucleus scattering. Some typical examples of inelastic heavy-1on scattering are also predicted successfully.

Some general properties of the folding model are reviewed and its theoretical basis is discussed. An explicit density-dependence is examined for one particular realistic interaction and found not to change the results. Single nucleon exchange is included in an approximate way and its importance is studied.

In addition to being a study of the folding model, this work also provides a systematic and comprehensive optical model analysis of heavy-1on elastic scattering in this energy range.

\section{Introduction}

The understanding of peripheral heavy-ion (HI) collision processes in general, and elastic and inelastic scattering in particular is an important part of an overall understanding of heavy-ion reactions. One of the most widespread approaches to this problem is based upon the use of an optical potential for the description of the elastic scattering of two heavy ions. Although HI scattering can be discussed and understood without reference to nuclear potentials (the recent review article by Frahn and Rehm [1] describes such an alternative), the optical model approach is both flexible and relatively familiar. It also provides a method for generating distorted waves for calculating other reaction processes. The optical model approach appears especially suitable for coupled channel formulations of HI reactions. While many of the systematics of HI scattering may be understood in terms of empirical parameterizations of the nuclear optical potential and its variation with bombarding energy, nucleon number etc., we feel that a satisfactory microscopic understanding of $\mathrm{HI}$ collisions should be founded on the underlying nucleon-nucleon $(\mathrm{N}-\mathrm{N})$ interaction. Within the framework of an optical model description of HI scattering this means calculating the nuclear optical potential from the $\mathrm{N}-\mathrm{N}$ interaction.

The most transparent relationship between the N-N interaction and HI optical potentials occurs when the double-folding model is applicable. In this model the optical potential for HI scattering is obtained by averaging an appropriate $\mathrm{N}-\mathrm{N}$ interaction over the matter distributions within the two colliding ions in the same way that the Coulomb interaction potential between two charge distributions is obtained by averaging the point-charge $r^{-1}$ interaction over the distributions.

Folding models, sometimes with higher-order corrections included, have been used for many years to generate potentials for use in nuclear scattering problems (see, for example, Sinha [2] and Barrett and Jackson [3]). This use has ranged from the purely phenomenological [4] all the way to sophisticated attempts to deduce the potential in an essentially parameter-free way from the nucleon-nucleon force $[5,6]$. Some early attempts to use this approach to deduce the interaction potential for two heavy ions (i.e. composite nuclei with $A>4$ ) from nucleon-nucleon scattering were made by Dover and Vary [7], Satchler [8] and Sinha [9]. Similar ideas underlay a potential model for bound cluster states in nuclei [10].

Our purpose here is to consider further the application of this approach to the scattering of 
two composite nuclei. We do not attempt to justify formally the use of a folding model for heavy-ion potentials. Rather, we compare with experiment the results of double-folding calculations using a "realistic" effective interaction which was recently derived [11] from $G$-matrix elements based upon the Reid nucleon-nucleon potential and upon the Sussex matrix elements [12]. We shall show that this procedure gives a good account of a representative sample of the available scattering data (with the exceptions of ${ }^{6} \mathrm{Li}$ and ${ }^{9} \mathrm{Be}$ ) which cover ions with energies up to about $20 \mathrm{MeV}$ per nucleon. We also discuss some of the features of folding models, giving careful consideration to the sources of uncertainties.

The "realistic" interaction used here has also been applied successfully to light-ion elastic and inelastic scattering [11]. It is desirable to have this work extended so as to provide a unified description which encompasses both light- and heavy-ion scattering and which is also firmly grounded on nucleon-nucleon scattering data. Further, the nucleus-nucleus potential is an object of fundamental importance not only for the description of elastic cross sections but also as an ingredient in the description of all the phenomena which occur when two nuclei collide. Consequently, the results of the present work could be of value for predicting the potential for those systems for which the actual elastic scattering is not known or is difficult to obtain.

\section{Heavy ion potentials}

Potentials appropriate for the elastic scattering of two heavy ions have been discussed by various authors (see, for example, [13-19] where other references may be found also).

The potential between two nuclei or heavy ions is not uniquely defined. Failure to remember this has sometimes led to confusion. A conventional optical model potential $U(R)$ for two nuclei $\mathrm{a}+\mathrm{A}$ is one which appears in a one-body Schrödinger equation

$$
\left[-\frac{\hbar^{2}}{2 \mu_{\alpha}} \nabla^{2}+U(R)\right] \chi(R)=E \chi(R)
$$

where $\mu_{\alpha}$ is the reduced mass of the pair, $R$ is the separation of their centers of mass and $E$ is the CMS energy of relative motion. The solution $\chi(R)$ with the appropriate boundary conditions describes the elastic scattering of a $+A$. One standard way to make it plausible that eq. (1) may adequately describe elastic scattering is to expand the total wavefunction of the a $+\mathrm{A}$ system in terms of the internal eigenstates of the separate nuclei,

$$
\Psi_{\alpha}=\sum_{i j} \psi_{\mathrm{a} i} \psi_{\mathrm{A} j} \chi_{i j}(\boldsymbol{R})
$$

where $\chi_{i j}(R)$ describes their relative motion with internal states labelled $i$ and $j$. Then $\chi_{00}$ gives the elastic scattering if $i, j=0$ denotes the ground state. If this term is projected out, following Feshbach [20], an exact equation for $\chi_{00}$ is obtained which is of the form (1) but with an effective potential operator $U_{\mathrm{op}}$. For the moment we ignore the effects of antisymmetrization between the two nuclei, although we assume the individual $\psi_{\mathrm{a} i}$ and $\psi_{\mathrm{A} j}$ themselves to be antisymmetric. Then $U_{\mathrm{op}}$ has the form

$$
\begin{aligned}
U_{\mathrm{op}} & =V_{00}+\sum_{\alpha \alpha^{\prime}} V_{0 \alpha}\left(\frac{1}{E-H+\mathrm{i} \varepsilon}\right)_{\alpha \alpha^{\prime}} V_{\alpha^{\prime} 0} \\
& =U_{\mathrm{F}}+\Delta U, \text { say }
\end{aligned}
$$


where $V$ is the interaction between a and $\mathrm{A}$, and the sum is over all the excited states of one or both nuclei. The first term is real and is simply the so-called folded potential,

$$
U_{\mathrm{F}}(R)=V_{00} \equiv\left(\psi_{\mathrm{a} 0} \psi_{\mathrm{A} 0}|V| \psi_{\mathrm{a} 0} \psi_{\mathrm{A} 0}\right),
$$

where the round brackets denote integration over the internal coordinates of the two nuclei. The remaining term $\Delta U$, which we may refer to as a dynamic polarization potential, arises from coupling to all the other states, is much more difficult to calculate, and in general is complex, non-local, energy- and angular-momentum dependent.

In phenomenological approaches it is this object $U_{\text {op }}$ which is approximated by a local, complex model potential $U(R)$, for example of the Woods-Saxon form. In this paper we explore instead the use of the first term $U_{\mathrm{F}}$ as an approximation to the real part of $U_{\mathrm{op}}$, using a "realistic" interaction $V$ and with the addition of a phenomenological imaginary potential to represent the absorptive effects of $\Delta U$.

A point we wish to stress is that with the potential $U_{\text {op }}$ (and by implication an equivalent model potential $U$ ) defined in this way, eq. (1) describes the relative motion of the two nuclei while they both remain in their respective ground states. This may be a very small component of the total wavefunction in that region of space where the two nuclei overlap appreciably; in that case the strong absorption into other channels manifests itself through $\chi_{00}(R)$ becoming small.

This is to be contrasted with most of the potentials calculated for heavy ion collisions [13-18; see also, for example, 21]. These calculations attempt to follow to a greater or lesser degree the readjustments that the two nuclei must make as they begin to interact and overlap. Such a potential energy function is not relevant just to the $\chi_{00}$ component of the wavefunction (2) but is to be used in a description of the motion of a wave packet which includes a wide range of excited states of the separated systems. It is not to be identified with $U_{\mathrm{op}}$ of eq. (3), and in principle it should not be used in an equation like eq. (1) without at least allowing for the corresponding changes in the kinetic energy term [22]. Further, there is an infinite number of such potential functions, each one corresponding to one or both of the nuclei in excited states when widely separated. Transitions between these potentials result in non-elastic scattering and hence absorption from the elastic channel. However, most calculations are performed in the adiabatic limit so that such transitions do not occur and their absorptive and other effects are ignored.

Although we prefer to define our potential a propos the specific component $\chi_{00}$ through eq. (3), we freely admit that the physical processes just discussed still manifest themselves through the dynamic polarization term $\Delta U$. This term certainly gives rise to the imaginary potential that is required and, in principle, also contributes to the real part. Our approach here is simply to see how successful is the use of $U_{\mathrm{F}}$ alone for the real part, at least in those regions of space which are important for the scattering. Recent crude estimates [23-25] have suggested that $\operatorname{Re} \Delta U \ll \operatorname{Im} \Delta U$ when calculated to second order in $V$, at least in the surface region.

Because of the strong, short-range nature of the nucleon-nucleon force, the $V$ in eq. (3) is not the bare interaction. As usual in nuclear model calculations, it is assumed that the bare interaction has been first transformed into an effective interaction ( $G$-matrix) and it is this which $V$ represents. This transformation itself incorporates some polarization effects, namely those due to short-range correlations between pairs of nucleons, and in principle allows us to use simpler nuclear wavefunctions such as those of the shell model. The present work uses an effective interaction [11] based upon a $G$-matrix derived from the Reid nucleon-nucleon potential.

Most of the available heavy ion scattering data is sensitive only to the tail of the nucleus-nucleus 
potential, in the vicinity of some strong absorption radius which is typically about $1.5\left(A_{1}^{1 / 3}+A_{2}^{1 / 3}\right) \mathrm{fm}$. In this region of small overlap it is perhaps not unreasonable that the folded potential $U_{\mathrm{F}}$ is a good approximation to the real potential. However, we shall discuss a few cases such as ${ }^{12} \mathrm{C}+{ }^{12} \mathrm{C},{ }^{40} \mathrm{Ca}+\alpha$ and ${ }^{40} \mathrm{Ca}+{ }^{6} \mathrm{Li}$ where the scattering does appear to probe the potential at much smaller radii where the two ions essentially overlap completely. This sensitivity to the interior has been explained $[26,27]$ in terms of the relatively weaker absorption in these cases.

A more complete test of a heavy ion potential would include its use in analyses of direct inelastic scattering and transfer reactions as well as elastic scattering. These may involve details of the wavefunction in the nuclear surface region to which the elastic scattering in itself is not so sensitive [28]. Some preliminary applications to inelastic scattering are reported here; however, our folded potentials have been used in only a few analyses of nucleon transfer reactions. Application [29] to transfers between ${ }^{12} \mathrm{C}$ and ${ }^{12} \mathrm{C}$ which are sensitive to the interior region led to inconclusive results, while their use [30] for the very peripheral transfers between ${ }^{11} \mathrm{~B},{ }^{12} \mathrm{C}$ and ${ }^{208} \mathrm{~Pb}$ showed no significant differences from the use of conventional Woods-Saxon potentials which were much shallower at small radii but similar in the surface.

Attempts are sometimes made [14-18] to relate the potentials required to describe elastic scattering of heavy ions to those inferred from fusion barriers. In most cases, fusion is believed to occur at smaller separations of the ions than are usually important for scattering. Numerous excitations are likely to have occurred by the time such close contact is established; consequently, the relative motion is no longer described simply by $\chi_{00}(R)$ and the corresponding potential energy function has been modified from the $U_{\text {op }}$ of eq. (3). The calculated potentials mentioned above which allow for readjustments of the ions are more likely to be appropriate for estimating fusion barriers. Asymptotically, these potentials assume the values given by the folding model with the corresponding interactions, so it is possible that consistency between fusion and elastic scattering can be obtained when, as usually happens, the scattering is only sensitive to the tail of the potential. Indeed, a recent [16] classical analysis of fusion cross sections resulted in a potential which at large separations is similar to that obtained from analysis of scattering data. A more recent [18] semi-empirical generalization of the liquid-drop model has also achieved a unified description of fusion and scattering (as well as fission). Nonetheless, we argue that these potentials will be inappropriate for scattering in those cases where the scattering is sensitive to the potential at smaller distances.

\section{The model}

\subsection{Double-folded potentials}

If $V$ is a local two-body operator,

$$
V=\sum_{i, j} v_{i j}
$$

where nucleons $i$ are in one nucleus and nucleons $j$ in the other, the folded potential (4) may be written

$$
U_{\mathbf{F}}(\boldsymbol{R})=\int \mathrm{d} \boldsymbol{r}_{1} \int \mathrm{d} \boldsymbol{r}_{2} \rho_{1}\left(\boldsymbol{r}_{1}\right) \rho_{2}\left(\boldsymbol{r}_{2}\right) v\left(\boldsymbol{r}_{12}=\boldsymbol{R}+\boldsymbol{r}_{2}-\boldsymbol{r}_{1}\right)
$$




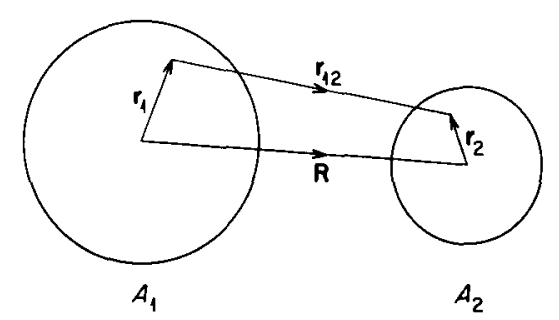

Fig. 1. Coordinates used in folding calculations.

Here $\rho_{i}$ is the distribution of the centers of mass of the nucleons in the ground state of the $i$ th nucleus (which we shall refer to as its "density distribution") while the coordinates are defined by fig. 1. Because there is integration over two densities, this is often called the double-folding model.

The expression (5) involves a six-dimensional integral. However, contrary to some suggestions [161], this is quite simple to evaluate. If we work in momentum space (see appendix B), it reduces to a product of three one-dimensional integrals (Fourier transforms) and often one or more of these need not be done explicitly if analytic forms for the Fourier transform exist. Even the construction of the densities themselves from the shell model (rather than using analytic models such as the Fermi distribution) is fast. The code we use will construct such a folded potential at all required radial points (typically 300 of them) in a few seconds using the IBM-360/91 computer.

The central part of the effective interaction may be written

$$
v_{12}=v_{00}\left(r_{12}\right)+v_{01}\left(r_{12}\right) \tau_{1} \cdot \tau_{2}+v_{10}\left(r_{12}\right) \sigma_{1} \cdot \sigma_{2}+v_{11}\left(r_{12}\right) \sigma_{1} \cdot \sigma_{2} \tau_{1} \cdot \tau_{2} .
$$

In general there will be spin-orbit and tensor terms also. When neither target nor projectile has spin, $U_{\mathrm{F}}$ becomes a function of the magnitude of $\boldsymbol{R}$ only and the $v_{S T}$ terms in eq. (6) with $S=1$ do not contribute. More generally, $U_{\mathrm{F}}$ will be non-spherical and include spin-dependent terms. Usually the spin terms are relatively unimportant for determining cross sections [31, 32] (see also appendix A). The non-spherical parts of $U_{\mathrm{F}}$, resulting from one or both of the densities $\rho_{i}$ being non-spherical, may give rise to inelastic scattering to collective rotational or vibrational states [33]. These non-spherical parts affect the elastic scattering of spin-zero nuclei only if a coupledchannel calculation is made; we do not do this here. Elastic scattering of nuclei with non-zero spin may be induced by the non-spherical parts of the potential [34], but in one comparison [35] of ${ }^{16} \mathrm{O}$ scattering from ${ }^{59} \mathrm{Co}$ and ${ }^{69} \mathrm{Ni}$ this effect was found to be negligible. Consequently, we restrict ourselves here to spherical densities and hence spherical potentials $U_{\mathrm{F}}$.

Isospin has not been referred to explicitly in eq. (5). If one or both nuclei have $N=Z$ (and hence zero isospin), then only $v_{S T}$ terms in eq. (6) with $T=0$ can contribute. Those with $T=1$ can contribute when both nuclei have $N \neq Z$. However, explicit evaluation for some typical cases (appendix A) shows these terms to be negligible for the interactions $v$ used here.

These various considerations lead us to evaluate the folding integral (5) with spherical densities and with the $v_{00}$ part of the effective interaction (6).

\subsection{Single-folded potentials}

We should note in passing that if only one of the integrations in eq. (5) is done, the result is a folded nucleon-nucleus potential. For example, we can rewrite eq. (5) as 


$$
U_{\mathrm{F}}(\boldsymbol{R})=\int \mathrm{d} \boldsymbol{r}_{1} U_{2 \mathrm{~N}}\left(\boldsymbol{R}-\boldsymbol{r}_{1}\right) \rho_{1}\left(\boldsymbol{r}_{1}\right)
$$

where

$$
U_{2 \mathrm{~N}}\left(\boldsymbol{R}-\boldsymbol{r}_{1}\right)=\int \mathrm{d} \boldsymbol{r}_{2} \rho_{2}\left(\boldsymbol{r}_{2}\right) v\left(\boldsymbol{r}_{12}\right)
$$

and

$$
r_{12}=R-r_{1}+r_{2}
$$

We shall briefly discuss later the use of $U_{2 \mathrm{~N}}$ to describe nucleon scattering from nuclei. However, the form (7a) has often been invoked but with the use of empirical, phenomenological nucleonnucleus potentials in place of $U_{2 \mathrm{~N}}[36]$. This so-called single-folding model invariably overestimates the strength of the real potential required by heavy-ion scattering data by a factor of about two [37].*

This is puzzling at first sight, since we shall see that the folded $U_{2 \mathrm{~N}}$ of eq. (7b), which is an ingredient of the heavy-ion potential (7a), is also moderately successful in reproducing nucleon scattering. Several explanations of this apparent paradox have been offered:

(i) When phenomenological nucleon potentials are used, they are generally taken to be of Woods-Saxon form. Now the long-range behaviour of $U_{\mathrm{F}}(R)$ is sensitive to the surface features of the potential substituted for $U_{2 \mathrm{~N}}$ in eq. (7a), and the Woods-Saxon form may not be an adequate representation. For example, it has been shown [38] that the square of a Woods-Saxon shape may give equally good fits to the nucleon scattering data but can result in a considerable reduction in the strength of the tail of the single-folded heavy-ion potential.

(ii) In general, the interaction $v$ is density-dependent; in the folding integral (5) it will depend upon the densities of both nuclei. An empirical nucleon-nucleus potential incorporates effects due to the density of one nucleus alone. When such a phenomenological potential is used in the single folding integral (7a), the effects due to the density of the other nucleus are neglected, whereas the potential $U_{2 \mathrm{~N}}$ of eq. (7b) remains implicitly dependent upon the density of nucleus 1 . This effect need not be negligible [39].

(iii) The phenomenological nucleon potential includes the effects of coupling to other channels [40] ( $\Delta U$ in eq. (3)) and these are likely to be quite different when the incident nucleon is bound in another nucleus compared to when it is free. Indeed, we shall see that our $U_{2 \mathrm{~N}}$, constructed according to eq. (7b), have some deficiencies compared to phenomenological potentials; in particular, they have somewhat too short ranges. This may reflect the neglect of contributions from $\Delta U$ to nucleon scattering, as well as the omission of an explicit density dependence.

\subsection{The effective interaction}

\subsubsection{Properties of the interaction}

The bare nucleon-nucleon interaction is too strong to be used as the $v$ which appears in the double folding integral (5). It has been popular to use for $v$ a simple potential, such as a Gaussian

\footnotetext{
* Reference [37] also indicated that the double-foldıng model predicted potentials which were two or more times too strong. We now understand, as the present paper explains, that this was due to deficiencies in the effective nucleon-nucleon interactions used in that work.
} 
[41] which fits low-energy nucleon-nucleon scattering. However, this has been shown [37] to overestimate the heavy ion potential by roughly a factor of two. (It has also been shown [42] to predict ( $\left.\mathrm{p}, \mathrm{p}^{\prime}\right)$ cross sections which are too large.)

We believe it is desirable to use an effective interaction $v$ which is based upon a realistic nucleonnucleon force, since one goal is to obtain a unified description of nucleon-nucleon, nucleonnucleus and nucleus-nucleus scattering. As a consequence, our effective interaction is some kind of $G$-matrix. A "high energy" approach to this problem was used by Dover and Vary $[7,33]$. In the high-energy limit (or impulse approximation) $v$ would become the (complex) $t$-matrix for free space nucleon-nucleon scattering. However, for the low-energy heavy-ion collisions of interest here, there are very large corrections to be made for the effects of the nuclear medium in which the two interacting nucleons are embedded (Pauli principle, off-shell propagation and Fermi motion of the nucleons [43]). As a consequence, in practice a phenomenological ansatz was adopted for the effective interaction. Originally [7] it was taken to be of zero range with an adjustable, complex, strength. Later this was generalized to a Gaussian form with an arbitrary range initially of $1 \mathrm{fm}$ [44] and later of $1.4 \mathrm{fm}[33,45]$. This choice has important consequences for inelastic scattering (see below).

The "low-energy" approach used here [11] assumes that the effective interaction $v$ is similar to the $G$-matrix for two nucleons bound near the Fermi surface. One consequence is that our $v$ is real. More recent work on nucleon scattering $[5,6]$ has yielded a complex $G$-matrix; however, it seems probable that the absorptive processes in heavy-ion scattering are very different so that we prefer to treat the imaginary potential phenomenologically.

In an earlier approach [46] we used the long-range parts of the even-state Hamada-Johnston potential, in the spirit of the Moszkowski-Scott separation method. Although this gave moderately good results for nucleon scattering $[42,46]$, it overestimated heavy-ion potentials by more than a factor of two $[8,37]$. Indeed, in some cases it did not yield an adequate fit to the data and this discrepancy could be traced [47] to the long range of the OPEP component in the force. This is
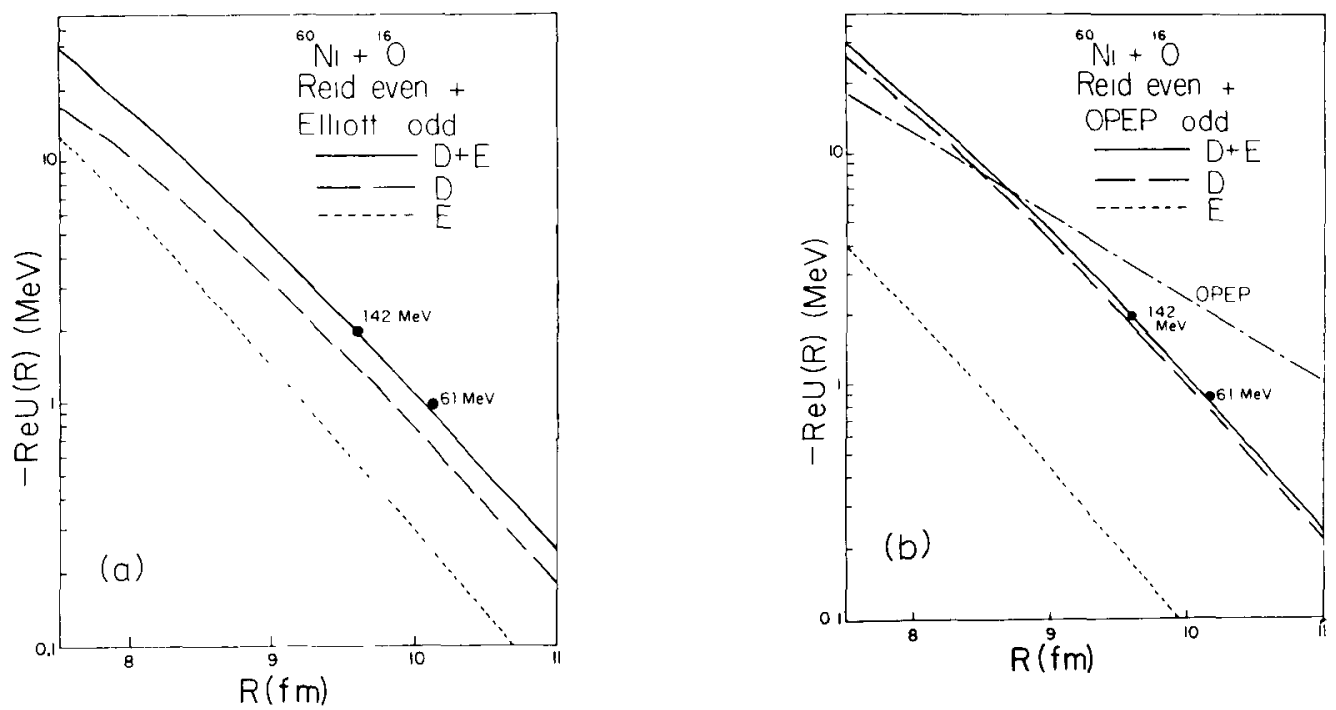

Fig. 2. Folded potentials near the strong absorption radii using the M3Y interactions (a) from eq. (10) and (b) from eq. (11). The dots are obtained from scattering data (see table 3). D stands for "direct", E stands for "exchange" (the contribution from the pseudopotential (15). The dash-dot curve is obtaned by using just the even-state part of the OPEP. 
illustrated for the ${ }^{16} \mathrm{O}+{ }^{60} \mathrm{Ni}$ system in fig. 2; the even-state OPEP term alone gives a large contribution to the potential near the strong absorption radius and has an unacceptably small slope. However, this OPEP component is spurious and arises because initially we took only the even-state interaction and ignored the odd-state parts. In a correct approach, at least the OPEP should be included in odd states. Then, in the combination [46] of even and odd parts which makes up $v_{00}$, the odd and even parts of the OPEP cancel so that this long-range component does not contribute. (The OPEP is purely $S=T=1$.)

The fact that the range of the effective interaction is extremely important is also illustrated by fig. 3. Folding calculations were made using either a single Gaussian or a single Yukawa for $v$ but with various ranges. In each case the strength was adjusted to give a potential of $-1.35 \mathrm{MeV}$ at $R=10.6 \mathrm{fm}$, as appropriate for ${ }^{40} \mathrm{Ca}+{ }^{40} \mathrm{Ca}$ scattering. Figure 3 shows the resulting volume integral of $v$,

$$
J=4 \pi \int v(r) r^{2} \mathrm{~d} r
$$

against its RMS radius. (The value $J=-400 \mathrm{MeV} \mathrm{fm}^{3}$ is roughly the value deduced from nucleonnucleus scattering.) For example, a zero-ranged force would require a value of $J$ that is about five times larger than one with $\left\langle r^{2}\right\rangle^{1 / 2}=2 \mathrm{fm}$. Further, we see that the choice of $1.4 \mathrm{fm}$ for the range of the Gaussian $[33,45]\left(\left\langle r^{2}\right\rangle^{1 / 2}=1.715 \mathrm{fm}\right)$ results in a "reasonable" value for $J$ of about $400 \mathrm{MeV}$ $\mathrm{fm}^{3}$, whereas the earlier [44] choice of $1 \mathrm{fm}\left(\left\langle r^{2}\right\rangle^{1 / 2}=1.225 \mathrm{fm}\right)$ requires $J \approx 720 \mathrm{MeV} \mathrm{fm}{ }^{3}$.

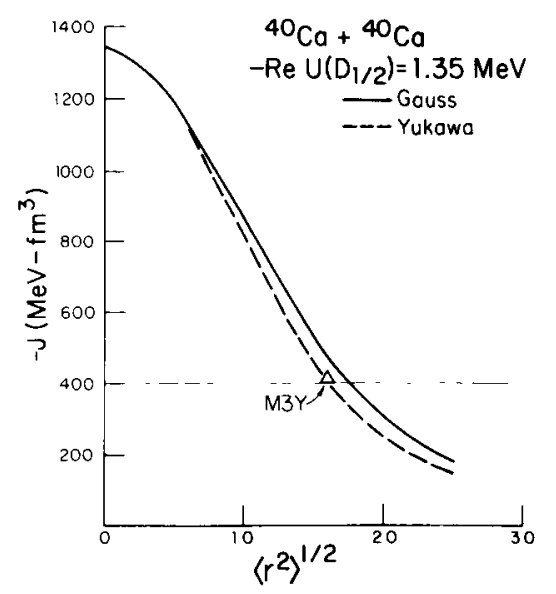

Fig. 3. Combinations of volume integral and range needed to give a potential of $-1.35 \mathrm{MeV}$ at $D_{1 / 2}=10.6 \mathrm{fm}$ when using a single Gauss or a single Yukawa term for the interaction.

Figure 3 also illustrates that, while $J$ and $\left\langle\boldsymbol{r}^{2}\right\rangle$ may be used to characterise the $v$ fairly well, the choice of its shape is not entirely unimportant. This shape-dependence may be studied further by expanding in the radial moments of $v$,

$$
U(R)=J\left[\int \rho_{1}\left(r_{1}\right) \rho_{2}\left(\left|\boldsymbol{R}-\boldsymbol{r}_{1}\right|\right) \mathrm{d} \boldsymbol{r}_{1}-\frac{1}{6}\left\langle r^{2}\right\rangle \int \nabla \rho_{1} \cdot \nabla \rho_{2} \mathrm{~d} \boldsymbol{r}_{1}+\ldots\right]
$$


where $\left\langle r^{2}\right\rangle$ is the MS radius of $v$. In the tail regions of interest, both $\rho_{1}$ and $\rho_{2}$ are falling exponentially and for typical realistic values of $\left\langle r^{2}\right\rangle$ the two terms in eq. (9) are comparable in magnitude. This is discussed further in appendix $\mathrm{B}$.

\subsubsection{The $M 3 Y$ interaction}

In this paper we are testing the effective interactions of Bertsch et al. [11] which are expressed as sums of three Yukawa terms; we call these M3Y. One Yukawa term was taken to be the OPEP; the second was chosen to have a range of $0.4 \mathrm{fm}$ which roughly simulates multiple pion exchange, and the third range of $0.25 \mathrm{fm}$ was chosen for computational convenience. The strengths of the latter two terms for the even-state $v$ were chosen to reproduce the $G$-matrix elements (in an oscillator basis) of the Reid soft-core potential. For the odd-state $v$, we use their fit to the matrix elements of Elliott et al. [12]. Full details are given by Bertsch et al. [11]. Then the $v_{00}$ component of eq. (6), which receives no contribution from the OPEP term, has the form

$$
v_{00}(r)=\left[7999 \frac{\mathrm{e}^{-4 r}}{4 r}-2134 \frac{\mathrm{e}^{-2.5 r}}{2.5 r}\right] \mathrm{MeV},
$$

and a volume integral of $J_{00}=-146 \mathrm{MeV} \mathrm{fm}^{3}$. On the other hand, if we assume that only the OPEP force acts in odd-states, we get instead

$$
v_{00}(r)=\left[6315 \frac{\mathrm{e}^{-4 r}}{4 r}-1961 \frac{\mathrm{e}^{-2.5 r}}{2.5 r}\right] \mathrm{MeV}
$$

with $J_{00}=-337 \mathrm{MeV} \mathrm{fm}^{3}$. It has been shown [48] that, in the folded potential, there is almost complete cancellation between the direct and exchange contributions arising from the short-range parts of the odd-state interaction which are included in eq. (10) (see fig. 2, for example). Consequently, the two interactions (10) and (11) are essentially equivalent for the construction of heavy ion potentials when exchange is included. Except when otherwise indicated, the calculations reported here use the form (10).

The potentials (10) and (11) are not as short ranged as their component Yukawas might suggest. The form (10) has $\left\langle r^{2}\right\rangle=7.26 \mathrm{fm}^{2}$, the same as a single Yukawa with a range of $1.10 \mathrm{fm}$ or a Gaussian with a range of $2.20 \mathrm{fm}$, while the potential (11) has $\left\langle r^{2}\right\rangle=3.11 \mathrm{fm}^{2}$. Further, Goldfarb [49] has shown that $50 \%$ of the folded potential near the strong absorption radius comes from $v(r)$ with $r \geq 1.6 \mathrm{fm}$.

Except for a weak dependence of the knock-on exchange terms (see below), the interactions (10) and (11) are independent of energy. Indeed, from their origin as fits to oscillator matrix elements, they represent an average over a certain range of energies. Further, these interactions are independent of the density of nuclear matter in which the two nucleons are embedded. Again, they represent an average of the interaction for densities ranging from zero to normal nuclear matter; considering the oscillator matrix elements upon which they are based suggests that they are representative of an average density of about $\frac{1}{3}$ of nuclear matter. This would seem not inappropriate for most direct scattering which largely occurs in the nuclear surface. Even for the very peripheral heavy ion scattering, the folding integral samples regions where the nuclear density has almost its full value. This is illustrated by fig. 4 which shows the variation of the potential for ${ }^{84} \mathrm{Kr}+{ }^{209} \mathrm{Bi}$ at three separations $R$ as the density $\rho(r)$ of each nucleus is cut off at some $r_{\max }=n a+c$ where $a=0.5 \mathrm{fm}$ is the density diffuseness parameter and $c$ is its half-density radius. (Simple Fermi shapes were 

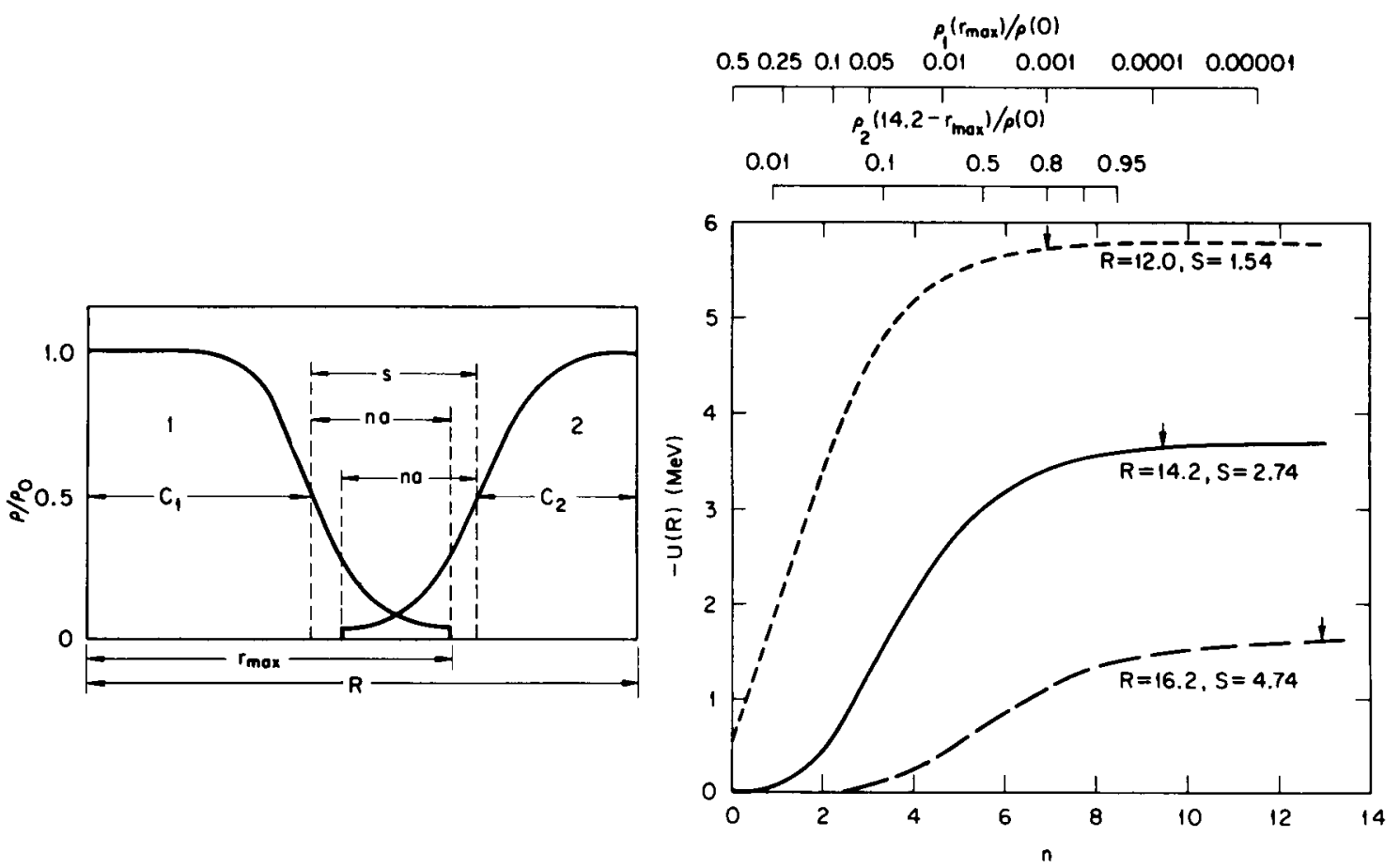

Fig. 4. Potential for the system ${ }^{84} \mathrm{Kr}+{ }^{209} \mathrm{Bi}$, at three distances $R$, when the densities for each are put to zero for $r_{\imath}>r_{\max }=c_{\imath}+n a$. The ratio of the density at this cut-off to the density in the interior is indicated by the uppermost scale. The scale below it shows the density of the other nucleus at the cut-off point for one nucleus, when $R=14.2$. Two-parameter Fermi shapes were used for the densities with $c_{1}=6.63 \mathrm{fm}, c_{2}=4.83 \mathrm{fm}$ and $a_{1}=a_{2}=0.5 \mathrm{fm}$; the left half of the figure shows a cut through these densities along the line of centers when these are separated by $R$. The potential curve for $R=16.2 \mathrm{fm}$ has been multiplied by 10 , that for $R=12.0 \mathrm{fm}$ was divided by 10 . The arrows indicate $99 \%$ of the full potential.

assumed for the densities with $c(\mathrm{Kr})=4.83 \mathrm{fm}, c(\mathrm{Bi})=6.63 \mathrm{fm}$.) The strong absorption radius for this system is about $R=14.2 \mathrm{fm}$, when the half-density points are separated by $5.5 a$. We see that at this separation, about $20 \%$ of the potential is still coming from where the tail of one density distribution is penetrating inside the half-density point of the other. To reach $1 \%$ accuracy for $R=14.2 \mathrm{fm}$, the cut-off must be displaced to where the density tail has fallen to $10^{-4}$ of the central value and this is probing the other nucleus into where its density has reached its full central value.

\subsubsection{Density-dependence and the DDD interaction}

In order to examine the possible importance of the explicit density dependence of the $G$-matrix, we made exploratory calculations using an effective interaction of Day et al. [50] which is also based upon the Reid soft-core potential. They define a local, trivially equivalent, $G$ by using the defining equation for the $G$-matrix, $G \phi=V \psi$ where $\phi$ is the uncorrelated (plane wave) relative motion wavefunction for the two nucleons and $\psi$ is the correlated one for two nucleons embedded in nuclear matter of density $\rho$. Also $V$ is a modified Reid potential. Then we define

$$
\bar{G}(r, \rho)=\overline{V(r) \psi(r, \rho) / \phi(r)}
$$

averaged over the relative momenta of the pair. We identify this $\bar{G}$ with our effective $v$ by using the local density approximation; the consequent density dependence of $v$ makes the integral in eq. (5) substantially more complicated. To simplify the calculations we parameterized the density dependence by an exponential form (see appendix $\mathrm{C}$ ) and in keeping with the frozen density approxima- 
tion, we took the density to be the sum $\rho=\rho_{1}\left(r_{1}\right)+\rho_{2}\left(r_{2}\right)$ of the densities at the respective positions of the two interacting nucleons. Hence in the folding (5) we take

$$
v\left(r_{12}\right)=\bar{G}\left(r_{12}, \rho=\rho_{1}\left(r_{1}\right)+\rho_{2}\left(r_{2}\right)\right) .
$$

This we call the DDD interaction. Again, the effects of single nucleon exchange were included in the way described in the next section.

The density-dependence of this DDD interaction is strongest in the triplet-even channel. At zero density, the DDD interaction (13) is much stronger than the M3Y, but it rapidly falls as the density increases and becomes comparable to M3Y for densities around 1/3 of normal density. Explicit calculations were made for a number of systems, including $\alpha+\alpha, \alpha+{ }^{40} \mathrm{Ca},{ }^{12} \mathrm{C}+{ }^{12} \mathrm{C}$, ${ }^{16} \mathrm{O}+{ }^{60} \mathrm{Ni},{ }^{40} \mathrm{Ca}+{ }^{40} \mathrm{Ca}$, and ${ }^{16} \mathrm{O}+{ }^{208} \mathrm{~Pb}$. These resulted in folded potentials which are a few per cent weaker for small $R$ but which agree within a few per cent near the strong absorption radius with those obtained by using the M3Y interactions. (This agreement again suggests that the latter are characteristic of an average density of about $1 / 3$ normal and that this average is appropriate for heavy-ion folded potentials.)

The recently developed density-dependent $G$-matrix of Brieva and Rook [6] is based upon the Hamada-Johnston potential. Calculations with this yield oscillator matrix elements which are very similar in magnitude and density dependence to those obtained with the DDD interaction. Further, the mean square radii of the important even-state components of the two interactions are similar: Hence, one would expect this more sophisticated interaction to result in folded potentials close to those obtained with the DDD one, and hence also close to those reported here using the M3Y interaction.

\subsubsection{Single-nucleon exchange}

The forms (4) and (5) do not explicitly show antisymmetrization between nucleons in different ions, although the individual nuclear wavefunctions $\psi_{\mathrm{a}}$ and $\psi_{\mathrm{A}}$ are themselves assumed to be antisymmetric. A general formalism has been developed [51] for exchange of nucleons between the two ions. A number of corrections are necessary [21, 52] when the nuclei have appreciable overlap. Unfortunately, most estimates (but see [53] for example) of these effects are made in an adiabatic limit and it is not clear how applicable these are to elastic scattering. Even near the strong absorption radius the relative angular kinetic energy is usually quite large. Moreover, there appears to be considerable cancellation amongst the various corrections [21]. More recently, exchange terms have been studied [54] within the framework of the resonating-group method. It was found that the most important contributions come from single-nucleon exchange (which we discuss in this section) and core exchange, that is, complete exchange of all the nucleons from the smaller system. Further, this latter term is only important when the two nuclei have almost the same number of nucleons $\left(A_{1} \approx A_{2}\right)$. The ranges of the exchange contributions to the potential decrease as the number of nucleons exchanged becomes larger. The presence of strong absorption by the imaginary potential will then further reduce the influence of these multi-nucleon exchange terms on the scattering.

As we have discussed, the dominant contributions to the potential near the strong absorption radius come from the surface and tail regions of the nuclear densities and the two densities do not overlap very much. Then single-nucleon exchange (SNE) between the two ions will be the leading correction arising from antisymmetrization. The SNE term that we consider is that in which the two interacting nucleons are interchanged; for nucleon-nucleus scattering, this has been called 
$[46,55]$ "knock-on exchange". This term is included formally in the double-folding integral (5) by replacing

$$
v_{12} \rightarrow\left(1-P_{12}\right) v_{12}
$$

where $P_{12}$ exchanges all the coordinates of nucleons 1 and 2 . At least two groups $[48,55,56]$ have estimated the importance of this SNE term for a common force and have found that it can be included quite accurately by replacing $-P_{12} v_{12}$ in eq. (14) by the pseudopotential

$$
\hat{J}(E) \delta\left(\boldsymbol{r}_{12}\right) \text {. }
$$

The strength $\hat{J}$ is considerably different for different spin, isospin $(S, T)$ components of the interaction (6), but it depends very weakly on the energy $E$ for energies between about 5 and $20 \mathrm{MeV}$ per nucleon. We have adopted the approximation (15) for the calculations reported here. The values of $\hat{J}$ were obtained by calibrating [55] against "exact" DWBA calculations of the SNE term in proton scattering. At $E=10 \mathrm{MeV}, \hat{J}_{00}=-262 \mathrm{MeV} \mathrm{fm}^{3}$ for the interaction (10) or $\hat{J}_{00}=-81 \mathrm{MeV} \mathrm{fm}{ }^{3}$ for the interaction (11). These values were used in all the heavy-ion cases discussed here since $\hat{J}$ varies by only a few per cent over the energy range covered.

Consequently, the effective interaction including SNE that is to be employed in the folding eq. (5) is

$$
v_{00}^{\prime}=v_{00}+\hat{J}_{00} \delta\left(\boldsymbol{r}_{12}\right) \text {. }
$$

With the $v$ of eq. (10), this $v^{\prime}$ has a volume integral of $J_{00}^{\prime}=-408 \mathrm{MeV} \mathrm{fm}^{3}$ and mean square radius of $\left\langle r^{2}\right\rangle=2.60 \mathrm{fm}^{2}$. Very similar values are obtained with a purely OPEP force in odd-states, $v$ of eq. (11); then we have $J_{00}^{\prime}=-418 \mathrm{MeV} \mathrm{fm}^{3}$ and $\left\langle r^{2}\right\rangle=2.51 \mathrm{fm}^{2}$. Except when indicated, the former version was used for the results reported here.

An alternate approximation for the SNE makes use of the Slater approximation to the one-body mixed density (see Sinha [2] for example). This also reduces to the use of a zero-range pseudopotential like eq. (15) except that the strength is now position-dependent [57]. This has also been tested against "exact" exchange calculations [57] and works quite well for nucleon elastic scattering. For heavy-ion scattering, with the interaction (10) used here, this approach predicts somewhat larger exchange terms than the pseudo-potential (15) so that the total heavy-ion potential may be increased by about $25 \%$ near the strong absorption radius. Of course, the Slater approximation itself is most suspect in the surface regions of the density which are important in this case. However, these results provide some measure of the uncertainty associated with the exchange terms and they also suggest that we may be somewhat underestimating the effects of SNE by using eq. (15).

\subsection{The nuclear densities}

\subsubsection{Densities from charge distributions}

The most direct measure we have of the densities $\rho_{i}\left(r_{i}\right)$ to be used in eq. (5) comes from electron scattering. This only yields information about the charge density and hence primarily about the proton distribution. However, for light nuclei $(A \lesssim 40$ ) with $N=Z$, it is not unreasonable to assume that the neutron and proton distributions are the same.

The finite size of the charge distribution of the proton itself [58] $\left(\left\langle r^{2}\right\rangle \approx 0.76 \mathrm{fm}^{2}\right)$ must be unfolded to give the distribution of the centers of mass of the protons. A further correction should be made for the charge distribution within the neutron $[59]\left(\left\langle r^{2}\right\rangle \approx-0.11 \mathrm{fm}^{2}\right)$. For this purpose, 
we may assume that the neutrons and protons are distributed similarly in the nucleus so that $\rho_{\mathrm{n}}=(N / Z) \rho_{\mathrm{p}}$; then the radii of the proton and charge distributions are related by [3]

$$
\left\langle r^{2}\right\rangle_{\mathrm{p}} \approx\left\langle r^{2}\right\rangle_{\mathrm{ch}}-0.76+0.11(N / Z) \text {. }
$$

This neutron correction is not necessarily negligible. For example, the potential for ${ }^{40} \mathrm{Ca}+{ }^{40} \mathrm{Ca}$ near the strong absorption radius was found to decrease by $8 \%$ when the densities used were obtained ignoring this correction.

Since the small momentum components are most important, it is convenient for this unfolding to assume that the average proton charge distribution has an exponential shape with a range such that $\left\langle r^{2}\right\rangle=0.76-0.11(N / Z)$ and this was done in our calculations.

\subsubsection{The neutron density}

Although we may assume that the neutron and proton distributions are the same in light nuclei when $N=Z$, it is not generally correct to assume that $\rho_{\mathrm{n}}=(N / Z) \rho_{\mathrm{p}}$ when $N>Z$. Indeed, there are indications [3] that the RMS radius of the neutrons is slightly greater than that for the protons when $N>Z$. The two triangles in fig. 5 illustrate for ${ }^{16} \mathrm{O}+{ }^{60} \mathrm{Ni}$ the effect of making two extreme assumptions about the neutron density of ${ }^{60} \mathrm{Ni}$; the lower one corresponds to taking $\rho_{\mathrm{n}}=(N / Z) \rho_{\mathrm{p}}$, while the upper results from assuming that $\rho_{\mathrm{n}}$ has the same central value as $\rho_{\mathrm{p}}$ (which requires the neutron half-density radius to be $0.205 \mathrm{fm}$ larger than the proton one). The latter assumption gives a potential $11 \%$ larger at the strong absorption radius than the former does. The effect, of course, can be larger when the neutron excess is larger. In the case of ${ }^{16} \mathrm{O}+{ }^{208} \mathrm{~Pb}$, for example, if we use $\rho_{\mathrm{n}}=(N / Z) \rho_{\mathrm{p}}$ with $\rho_{\mathrm{p}}$ being the Hartree-Fock proton density [60], instead of using the corresponding Hartree-Fock neutron density, the potential near the strong absorption radius is reduced by $35 \%$. This raises the interesting possibility that, once the validity of an effective interaction

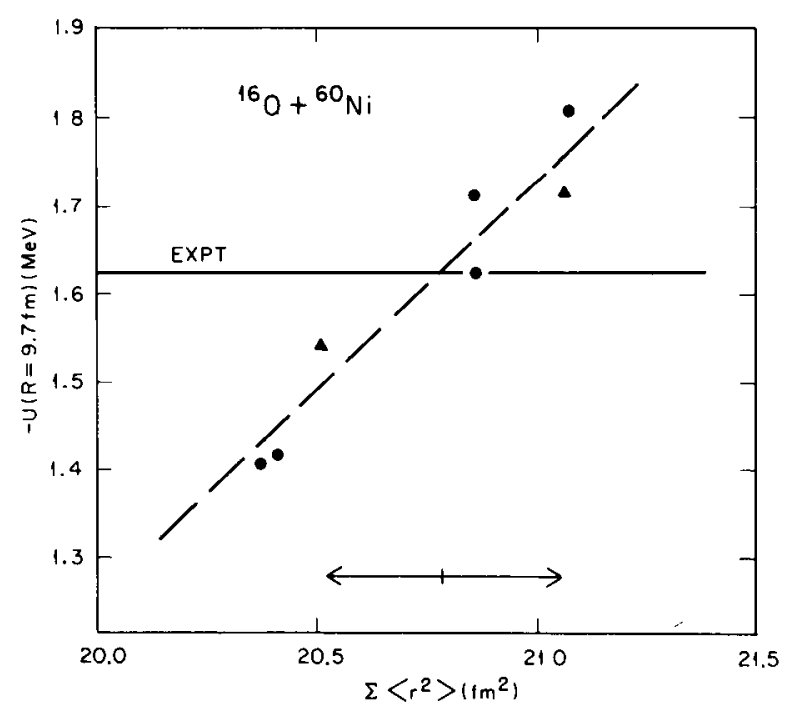

Fig. 5. The calculated potential at $R=9.7 \mathrm{fm}$ for a variety of choices of density distributions for ${ }^{16} \mathrm{O}$ and ${ }^{60} \mathrm{Ni}$ plotted against the sum of the mean square radii for those distributions. The dashed line is simply to guide the eye; the horizontal line is the value of $\operatorname{Re} U(R=9.7)$ required to fit the data. The arrows indicate the uncertainty in $\left\langle r^{2}\right\rangle$ allowed by the range of values quoted for the charge radius of ${ }^{60} \mathrm{Ni}$. As an example, the two triangles denote calculations in which the density of ${ }^{60} \mathrm{Ni}$ was assumed to have a two-parameter Fermi form; the lower value results from assuming $\rho_{\mathrm{n}}=(N / Z) \rho_{\mathrm{p}}$ while the upper one comes from taking $\rho_{\mathrm{n}}(r=0)=\rho_{\mathrm{p}}(r=0)$. 
has been established, the extremely peripheral nature of heavy-ion scattering may be a sensitive probe of the extent of neutron distributions in nuclei $[3,61]$.

We adopted the independent particle model (IPM) as a way to obtain reasonable neutron density distributions when $N \neq Z$.

\subsubsection{Hartree-Fock densities}

Hartree-Fock (HF) theory provides an IPM with a self-consistent potential derived from realistic nucleon-nucleon forces. We have chosen to use the matter densities obtained by Negele [60] for ${ }^{40} \mathrm{Ca},{ }^{48} \mathrm{Ca},{ }^{90} \mathrm{Zr}$ and ${ }^{208} \mathrm{~Pb}$ since his proton densities are in agreement with data for electron scattering, etc. (The appropriate center of mass recoil correction was applied using the oscillator approximation.)

\subsubsection{Shell-model densities}

Hartree-Fock densities are available only for a few doubly-magic nuclei. The more phenomenological shell model has the advantage of flexibility; it may be used for nuclei with unclosed shells and is easily extended to odd nuclei. Further, if the binding energies are chosen appropriately, there is a good chance of getting correctly the shape of the tail of the density distribution [62]. Against this is the uncertainty in the parameters to be used.

We chose the IPM potential to be local and of Woods-Saxon shape (with a spin-orbit coupling term of the usual Thomas form),

$$
U(r)=-V\left(\mathrm{e}^{x}+1\right)^{-1}, \quad x=(r-R) / a
$$

with

$$
R=r_{0}(A-1)^{1 / 3}
$$

For protons, we added the Coulomb potential due to a uniform charge distribution of magnitude $(Z-1) e$ and radius $r_{\mathrm{c}}(A-1)^{1 / 3}$. We used $r_{\mathrm{c}}=1.2 \mathrm{fm}$ except for the lightest nuclei for which $r_{\mathrm{c}}=1.35 \mathrm{fm}$ was used. We demanded that the resulting proton distribution be in agreement with electron scattering data, at least to the extent of having the correct mean square radius. (In view of the uncertainties in the neutron distribution, a more detailed fit [63] to electron scattering was not attempted.) Except for the lightest nuclei, we found that the Woods-Saxon potential parameters of Batty and Greenlees [64] $\left(V=60 \mathrm{MeV}, r_{0}=1.28 \mathrm{fm}, a=0.76 \mathrm{fm}\right)$ gave satisfactory values for the proton RMS radii. They also resulted in neutron RMS radii which were slightly larger by reasonable amounts [3] when the well depths were adjusted to reproduce approximately the known separation energies of the least-bound neutrons $\left(V=54 \mathrm{MeV}\right.$ for ${ }^{60} \mathrm{Ni}, 50 \mathrm{MeV}$ for ${ }^{88} \mathrm{Sr}$ and $45 \mathrm{MeV}$ for ${ }^{142} \mathrm{Nd}$ ). Consequently, we used these parameters for the neutron potentials also, except in the case of ${ }^{208} \mathrm{~Pb}$ where we used parameters which had been found [30] to give satisfactory fits to neutron pick-up data $\left(V=45 \mathrm{MeV}, r_{0}=1.25 \mathrm{fm}, a=0.65 \mathrm{fm}\right.$ ). (In principle, we should always be guided by neutron pick-up data, although in practice for most nuclei there are sufficiently large uncertainties in the values expected for the spectroscopic factors that the constraints imposed in this way are not very strong.) These potentials also give densities for the doubly-magic nuclei which are very similar to the HF ones of Negele; in the case of ${ }^{208} \mathrm{~Pb}$, potentials calculated using the $\mathrm{HF}$ and the shell-model densities agreed to about $1 \%$. (On the other hand, we also tried the Batty-Greenlees potential for the neutrons in ${ }^{208} \mathrm{~Pb}$, with $V=45 \mathrm{MeV}$ except for the last-filled shell where the value of $V$ was adjusted slightly to give the observed separation energies. This 
results in a larger neutron RMS radius of $5.793 \mathrm{fm}$, or $0.29 \mathrm{fm}$ larger than the proton RMS radius. We shall see later that this gives somewhat more satisfactory results for some heavy-ion scattering even though the neutron radius is appreciably larger than predicted by Hartree-Fock calculations $[3,60]$ or deduced from $1 \mathrm{GeV}$ proton scattering [65].)

Analyses of electron scattering from ${ }^{28} \mathrm{Si}$ and ${ }^{32} \mathrm{~S}$ have been made [66] using shell-model proton densities. We took these proton potentials and orbit occupancies and assumed that the neutron and proton densities were equal for these nuclei.

For the light nuclei ${ }^{12} \mathrm{C}$ and ${ }^{16} \mathrm{O}$ we used binding energies $[A /(A-1)]^{2}$ times the measured separation energies to account for $\mathrm{CM}$ recoil effects and thus ensure the correct behaviour in the tails [62]. We assumed a potential diffuseness of $a=0.65 \mathrm{fm}$ and adjusted the potential radius to give the desired radius for the proton distribution. We found $r_{0}=1.243 \mathrm{fm}\left({ }^{12} \mathrm{C}\right)$ or $1.367 \mathrm{fm}\left({ }^{16} \mathrm{O}\right)$. The results reported here were then obtained assuming that the neutron distribution was equal to that of the protons. However, we also studied the effects of assuming that the neutron and proton potentials were the same (except for a slight adjustment in depth to reproduce the observed separation energies) instead of the assumption that $\rho_{\mathrm{n}}=\rho_{\mathrm{p}}$. This prescription results in neutron distributions with RMS radii smaller than those for the protons by 1 to $2 \%$. The corresponding folded potentials are shallower by about twice this amount, but this kind of uncertainty is negligible in the present context.

For the odd nuclei ${ }^{10} \mathrm{~B},{ }^{11} \mathrm{~B}$ and ${ }^{13} \mathrm{C}$ we assumed that the densities were exactly as for ${ }^{12} \mathrm{C}$ except for the addition or subtraction of the appropriate $1 \mathrm{p}$ orbitals calculated in the same potential that was used for ${ }^{12} \mathrm{C}$. The density for ${ }^{15} \mathrm{~N}$ was treated as a $1 \mathrm{p}_{1 / 2}$ hole in ${ }^{16} \mathrm{O}$ in the same way. The heavier odd nuclei were related to the adjacent even nuclei in a like manner. There is no guarantee that this procedure is correct, although it is suggested by the Hartree-Fock picture for closed-shell \pm 1 -nucleon systems. Unfortunately, even the proton distributions are not well determined (by electron scattering) for most of the odd light nuclei.

The special case of ${ }^{6} \mathrm{Li}$ and ${ }^{9} \mathrm{Be}$ are discussed separately in a later section.

In principle, shell model densities should be corrected for recoil of the center of mass. This may be done in the harmonic oscillator approximation (and was in the cases of ${ }^{28} \mathrm{Si}$ and ${ }^{32} \mathrm{~S}$ ) or by the prescription based upon Clement [62]. We used the latter for the lightest nuclei, although the effects of neglecting recoil were quite negligible provided the shell model potential was readjusted to give a $\rho$ with the same $\left\langle r^{2}\right\rangle$ value. Recoil effects were ignored for heavier nuclei, where they are small anyway.

The RMS radii of the various density distributions are listed in table 1.

\subsubsection{Contributions from the major shells}

An interesting question is: how large are the contributions to the folded potential from the lastfilled major shells in the shell model densities? This was studied for the shells of the $\mathrm{Pb}$ nucleus in the ${ }^{208} \mathrm{~Pb}+{ }^{16} \mathrm{O}$ system. The fraction of the folded potential near the strong absorption radius $\left(\boldsymbol{R} \approx 12.5 \mathrm{fm}\right.$ ) due to the last-filled major shell of protons in ${ }^{208} \mathrm{~Pb}$ ( 5 orbits, 32 protons) is $17 \%$, while $46 \%$ is due to the last-filled major shell of neutrons ( 6 orbits, 44 neutrons). The predominance of the neutrons over the protons is due to their greater spatial extension (RMS radius of $6.10 \mathrm{fm}$ compared to $5.80 \mathrm{fm}$ for the protons) with a longer tail to their distribution. This emphasizes again the possibility of using heavy-ion scattering as a probe of neutron distributions.

The remaining $37 \%$ of the potential results from the other 132 nucleons which are more deeply 
Table 1

RMS radii for the density distributions used in the folding calculations

\begin{tabular}{|c|c|c|c|c|c|c|c|c|c|c|c|}
\hline Nucleus & ${ }^{4} \mathrm{He}$ & ${ }^{6} \mathrm{Li}$ & ${ }^{7} \mathrm{Li}$ & ${ }^{10} \mathrm{~B}$ & ${ }^{11} \mathrm{~B}$ & ${ }^{12} \mathrm{C}$ & ${ }^{13} \mathrm{C}$ & ${ }^{14} \mathrm{~N}$ & ${ }^{15} \mathrm{~N}$ & ${ }^{16} \mathrm{O}$ & \\
\hline$\left\langle r^{2}\right\rangle_{\mathrm{n}+\mathrm{p}}^{1 / 2}(\mathrm{fm})$ & 1.461 & 2.422 & 2.373 & 2.274 & 2.279 & 2.314 & 2.368 & 2.423 & 2.580 & 2.596 & \\
\hline$\left\langle r^{2}\right\rangle_{\mathrm{n}}^{1 / 2}(\mathrm{fm})$ & - & - & 2.441 & - & 2.283 & - & 2.414 & - & 2.596 & - & \\
\hline$\left\langle r^{2}\right\rangle_{\mathrm{p}}^{1 / 2}(\mathrm{fm})$ & 1.461 & 2.422 & 2.279 & 2.274 & 2.274 & 2.314 & 2.314 & 2.423 & 2.564 & 2.596 & \\
\hline$\left\langle r^{2}\right\rangle_{\mathrm{ch}}^{1 / 2}(\mathrm{fm})$ & 1.670 & 2.553 & 2.409 & 2.413 & 2.408 & 2.450 & 2.446 & 2.554 & 2.685 & 2.718 & \\
\hline Nucleus & ${ }^{17} \mathrm{O}$ & ${ }^{27} \mathrm{Al}$ & ${ }^{28} \mathrm{Si}$ & ${ }^{32} \mathrm{~S}$ & ${ }^{40} \mathrm{Ca}$ & ${ }^{44} \mathrm{Ca}$ & ${ }^{48} \mathrm{Ca}$ & ${ }^{58} \mathrm{Ni}$ & ${ }^{59} \mathrm{Co}$ & ${ }^{60} \mathrm{~N}_{1}$ & \\
\hline$\left\langle r^{2}\right\rangle_{n+p}^{1 / 2}(\mathrm{fm})$ & 2.666 & 2.987 & 3.001 & 3.148 & 3.366 & 3.410 & 3.566 & 3.723 & 3.753 & 3.760 & \\
\hline$\left\langle r^{2}\right\rangle_{n}^{1 / 2}(\mathrm{fm})$ & 2.727 & 3.001 & - & - & 3.345 & 3.472 & 3.659 & 3.734 & 3.788 & 3.788 & \\
\hline$\left\langle r^{2}\right\rangle_{\mathbf{p}}^{1 / 2}$ & 2.596 & 2.973 & 3.001 & 3.148 & 3.386 & 3.333 & 3.431 & 3.710 & 3.711 & 3.729 & \\
\hline$\left\langle r^{2}\right\rangle_{\mathrm{ch}}^{1 / 2}(\mathrm{fm})$ & 2.718 & 3.079 & 3.107 & 3.250 & 3.481 & 3.426 & 3.518 & 3.796 & 3.795 & 3.813 & \\
\hline Nucleus & ${ }^{88} \mathrm{Sr}$ & ${ }^{89} \mathrm{Y}$ & ${ }^{90} \mathrm{Zr}$ & ${ }^{124} \mathrm{Sn}$ & ${ }^{142} \mathrm{Nd}$ & ${ }^{207} \mathrm{~Pb}$ & ${ }^{208} \mathrm{~Pb}$ & ${ }^{208} \mathrm{~Pb}^{\mathrm{a})}$ & ${ }^{209} \mathrm{Bi}$ & ${ }^{209} \mathrm{Bi}^{\mathrm{b})}$ & ${ }^{208} \mathrm{~Pb}^{\mathrm{c})}$ \\
\hline$\left\langle r^{2}\right\rangle_{n+p}^{1 / 2}(f m)$ & 4.250 & 4.251 & 4.244 & 4.686 & 5.013 & 5.603 & 5.573 & 5.605 & 5.608 & 5.576 & 5.679 \\
\hline$\left\langle r^{2}\right\rangle_{\mathrm{n}}^{1 / 2}(\mathrm{fm})$ & 4.329 & 4.329 & 4.299 & 4.734 & 5.119 & 5.669 & 5.651 & 5.673 & 5.673 & 5.651 & 5.793 \\
\hline$\left\langle r^{2}\right\rangle_{\mathrm{p}}^{1 / 2}(\mathrm{fm})$ & 4.136 & 4.148 & 4.175 & 4.615 & 4.863 & 5.499 & 5.451 & 5.499 & 5.506 & 5.459 & 5.499 \\
\hline$\left\langle r^{2}\right\rangle_{\mathrm{ch}}^{1 / 2}(\mathrm{fm})$ & 4.210 & 4.222 & 4.249 & 4.679 & 4.926 & 5.553 & 5.505 & 5.552 & 5.560 & 5.513 & 5.552 \\
\hline
\end{tabular}

The densities for ${ }^{40,48} \mathrm{Ca},{ }^{90} \mathrm{Zr}$ and ${ }^{208} \mathrm{~Pb}$ are from Hartree-Fock calculations [60], the ${ }^{4} \mathrm{He}$ density was taken to be Gaussian and the remainder were constructed using the shell model, as discussed in the text. Where the neutron entries are left blank, it was assumed that neutron and proton densities were identical.

a) Shell-model densities for ${ }^{208} \mathrm{~Pb}$ upon which those for ${ }^{207} \mathrm{~Pb}$ and ${ }^{209} \mathrm{Bi}$ are based: see text.

${ }^{b)}$ Hartree-Fock density [60] for ${ }^{208} \mathrm{~Pb}$ plus one $1 \mathrm{~h}_{9 / 2}$ shell-model proton.

c) Shell-model density with protons as in case a) but neutrons in a well of the same size as the protons; see text.

bound in ${ }^{208} \mathrm{~Pb}$; these are more confined spatially (the RMS radius of this "core" is $5.38 \mathrm{fm}$ compared to $5.97 \mathrm{fm}$ for the sum of the last-filled shells).

\subsection{General features}

\subsubsection{Shape and strength of the potential}

The folded potential is, of course, very deep for small separations of the ions $(R \approx 0)$. If one ion is much smaller than the other, $A_{1} \ll A_{2}$ say, the central depth is approximately equal to $A_{1} J_{00}^{\prime} \rho_{2}\left(r_{2} \approx 0\right)$, or about $65 A_{1} \mathrm{MeV}$ if the central density is $\rho_{2} \approx 0.16$ nucleons $\mathrm{fm}^{-3}$. The potential is less deep when the ions are of comparable size, but this estimate gives the depth to within a factor of 2 even when $A_{1}=A_{2}$. We return later to the question whether this potential in the interior is significant and whether information about it can be deduced from experiment.

Of particular importance is the fact that a folded potential does not have the same shape as the Woods-Saxon form commonly used. Indeed, it has an overall shape closer to that of the WoodsSaxon form raised to some power $v$, with $v \gtrsim 2$ (see $[67,68,72]$, for example). This difference is relevant when the scattering does probe the potential inside the strong absorption radius and hence is sensitive to its shape at least in the surface region. Such is the case for high-energy ( $\gtrsim 100$ $\mathrm{MeV}$ ) alpha scattering for which shapes differing from the Woods-Saxon one have been found to give superior fits to data [69]. (However, we shall see later that these data also seem to demand a potential which is more shallow than given by our interaction.) Instances of sensitivity to the 
interior potential also occur at lower energies when the absorption is "anomalously" low, and these have also been fitted using potentials of folded or similar shape [70-73]. These shapes have also been found superior for calculations of cluster-type bound states in nuclei $[10,74]$.

Further, the tail of the folded potential does not decrease like a simple exponential [49], as would be the case for the Woods-Saxon type potentials, the global potential introduced by Christensen and Winther [19] or the approximate form of the proximity potential [75]. Over a limited range, for $s>1$, the shape of the folded potential $U(R)$ may be represented by $s^{n} \exp (-s / \beta)$ where $s=R-R_{1}-R_{2}$ is the separation between the surfaces of the two nuclei. For example, in the important range of $R \approx 8$ to $11 \mathrm{fm}$ (or $s \approx 1$ to $4 \mathrm{fm}$ ) for ${ }^{16} \mathrm{O}+{ }^{60} \mathrm{Ni}$ we have $n \approx 1$ and $\beta \approx 0.534 \mathrm{fm}$ if we take $R_{1}+R_{2}=6.8 \mathrm{fm}$. In most cases the scattering of heavy ions is sensitive only to the potential for a very restricted range of $r$ values around the strong absorption radius, so only two quantities, the value and slope in this region, are important. Any parameterization (within reason) which reproduces these two quantities will yield equivalent fits to the data. In this circumstance the inverse of its logarithmic derivative at this radius becomes a significant quantity. We shall see (table 3) that this has values between 0.6 and $0.7 \mathrm{fm}$ to be compared with the $0.63 \mathrm{fm}$ adopted by Christensen and Winther [19] for their empirical potential and the nominal value of $0.75 \mathrm{~b}=0.75 \mathrm{fm}$ used by Blocki et al. [75].

The strength of the folded potential in its tail, for a given separation $s$ between the surfaces of the two nuclei, varies with the sizes of the two nuclei in the way suggested by the proximity theorem [75], namely it is approximately proportional to the harmonic mean of their radii of curvature,

$$
\bar{R}=R_{1} R_{2} /\left(R_{1}+R_{2}\right) \text {. }
$$

Consequently, it is roughly proportional to

$$
\left(A_{1} A_{2}\right)^{1 / 3} /\left(A_{1}^{1 / 3}+A_{2}^{1 / 3}\right) \text {. }
$$

However, care must be taken when applying this rule-of-thumb. The various calculated $U_{\mathrm{F}}(R)$ do not all vary with $s$ in exactly the same way for large $R$; in particular, as we have stressed, the dependence on $s$ is not simply exponential. Further, the proximity result applies only if the radii $R_{i}$ are defined appropriately [75] and, in any case, is itself approximate [76].

\subsubsection{Effects of single-nucleon exchange}

As discussed above, the effects of single nucleon exchange were included by use of the pseudopotential of eq. (16). Since $\hat{J}_{00}$ is negative for both the interactions (10) and (11), the result is to make the potential more attractive. (See also [77] for similar results obtained with a different interaction.) The net effect is small near the strong absorption radius, as illustrated by fig. 2 . The precise division into direct (D) and exchange (E) terms depends upon the choice made for the interaction in odd states; however, the total $(D+E)$ is insensitive to this choice (provided the correct OPEP components are included). As shown by table 2, this is because the non-OPEP parts of the odd-state forces have short ranges and their direct and exchange contributions to the folding almost completely cancel. For the interaction (10), the cancellation between these terms is complete to better than $3 \%$ in the tail of the potential for ${ }^{16} \mathrm{O}+{ }^{60} \mathrm{Ni}$, for example (table 2), so that they contribute less than $1 \%$ of the total potential in this region. The cancellation is less complete for smaller $R$ values, but it is still very substantial. Consequently, it is a good approximation to neglect the non-OPEP parts of the odd-state effective interaction. This is a fortunate situation because these parts of the interaction appear to be the least well understood. 
Table 2

Direct (D) and exchange (E) parts of the folded potentıal for ${ }^{16} \mathrm{O}+{ }^{60} \mathrm{Ni}$ at $R=9.6 \mathrm{fm}[48]$

\begin{tabular}{|c|c|c|c|c|c|}
\hline Interaction & type $^{\text {a) }}$ & $\mathrm{D}$ & E & $D+E$ & $(D+E) / D$ \\
\hline Even & Odd & $(\mathrm{MeV})$ & $(\mathrm{MeV})$ & $(\mathrm{MeV})$ & \\
\hline Reid & Elliott & -1.41 & -0.56 & -1.97 & 1.40 \\
\hline Reid & OPEP & -1.79 & -0.17 & -1.96 & 1.10 \\
\hline - & (Elliott-OPEP) & -0.38 & +0.39 & +0.01 & -0.03 \\
\hline
\end{tabular}

a) Reid + Elliott corresponds to the interaction of eq. (10), while Reid + OPEP corresponds to eq. (11). (Elliott-OPEP) consists of the short-range (non-OPEP) part of the odd-state force.

The exchange term becomes relatively larger at smaller distances $R$; for the ${ }^{16} \mathrm{O}+{ }^{60} \mathrm{Ni}$ case, $\mathrm{E} / \mathrm{D} \rightarrow 0.22$ in the interior for the Reid-even plus OPEP-odd interaction (11), while $\mathrm{E}>\mathrm{D}$ for the Reid-even plus Elliott-odd interaction (10) for $R<7 \mathrm{fm}$. However, as just remarked, there is still nearly complete cancellation between direct and exchange from the non-OPEP odd force, so that the two interactions still give almost the same potential even for small $R$.

The relative importance of exchange at large $R$ will increase for heavier systems because their surfaces have less curvature and because the equivalent exchange interaction is of much shorter range (zero range with our model (15)) than the direct interaction (10) or (11). However, this effect is small. For example, model calculations for ${ }^{12} \mathrm{C}+{ }^{12} \mathrm{C}$ and ${ }^{208} \mathrm{~Pb}+{ }^{208} \mathrm{~Pb}$ with the Reid-even plus Elliott-odd force showed that, for a separation $R=R_{1}+R_{2}+3 \mathrm{fm}$, the exchange contribution was $20 \%$ for the light system but increases to $26 \%$ for the heavy system. Allowing the exchange pseudo-potential to have a finite range would make this increase even smaller.

The strength $\hat{J}(E)$ of the pseudo-potential (15) varies with bombarding energy but the variation is small over the energy range $E / A \approx 5$ to $20 \mathrm{MeV}$ of interest here. The interaction (10), for example, is associated with

$$
\hat{J}_{00}(E) \approx 276(1-0.005 E / A) \mathrm{MeV} \mathrm{fm}^{3}
$$

which imparts a very weak energy dependence to the folded potential. It is consistent to ignore this since we ignore any variation with $E$ of the effective interaction $v$ itself.

Finally, it should be remarked that application of the Slater approximation to the exchange term [57] results in larger estimates of this contribution than does the pseudo-potential (15). For example, in calculations [57] for ${ }^{40} \mathrm{Ca}+{ }^{40} \mathrm{Ca}$, which would be typical, the Slater approach with the interaction (10) yielded a (direct + exchange) potential at the strong absorption radius (about $10.6 \mathrm{fm}$ ) of $-1.5 \mathrm{MeV}$ which is $25 \%$ larger than the value $-1.2 \mathrm{MeV}$ obtained by use of the zero-range pseudo-potential (15). This difference would be much less for the interaction (11) simply because the overall magnitude of the exchange terms is greatly reduced. On the other hand, a force with stronger odd-state components would enhance the difference. It is difficult to evaluate these two approaches to exchange until exact calculations are available, although the results might suggest (see also [56]) that we somewhat underestimate the exchange contribution by using the zero-range approximation (15). In any case, the uncertainty involved is not much outside the limit to our confidence in the folding model and its attendant uncertainties. 


\subsubsection{Potential barriers and fusion}

We have already expressed our opinion that the potential which governs the wave function of relative motion $\left(\chi_{00}(R)\right.$ in section 2$)$ and describes elastic scattering is not everywhere the same as the potential which may be appropriate for describing fusion cross sections. Fusion may occur following fast (direct) transitions to non-elastic channels as well as from the entrance channel itself. These "doorway" effects will influence the potential function which describes the evolution of the system towards fusion in ways different from their contributions to the polarization potential $\Delta U$ for elastic scattering. Consequently, we can expect the two potentials to differ when the two nuclei begin to make contact. One manifestation of this is that while the S-wave barrier heights deduced [17] are similar to those obtained with our folded potentials, the empirical barrier position generally occurs at a smaller radius (by amounts up to about $1 \mathrm{fm}$ ). This implies that the "fusion potential" has a less steep slope in the surface region than our folded potentials which fit elastic data. This is confirmed by comparison with the universal empirical potential of Bass [16] whose slope is similar to that of the folded potential in the vicinity of the strong absorption radius but which becomes less steep at smaller radii; at $R \simeq R_{1}+R_{2}$, or $s \approx 0$, the logarithmic derivative of this empirical potential is about one half that for the folded potentials. The potential of Krappe et al. [18] shows a similar but less marked difference.

\section{Application to nucleon scattering}

The volume integral per nucleon of the real part of the optical potential for neutrons and protons appears to be determined quite accurately from elastic scattering data $[78,79]$. This is equal to the volume integral of the effective interaction, eq. (8), for a folded potential provided the interaction is density-independent. The empirical values for the $(S, T)=(0,0)$ part extrapolated to a proton energy of $10 \mathrm{MeV}$ are given by $[78,79]$

$$
J_{00}^{\prime}(\text { expt. }) \approx-(430 \pm 20) \mathrm{MeV} \mathrm{fm}^{3}
$$

which agree quite well with the values -408 and $-418 \mathrm{MeV} \mathrm{fm}^{3}$ for the $\mathrm{M} 3 \mathrm{Y}$ interactions (10) and (11), respectively, when exchange is included. When the target nuclei have $N>Z$ we also need the effective interaction $v_{01}$ for $(S, T)=(0,1)$ (see eq. (6)). This was based upon the same Reid-even plus Elliott-odd force that gives the $v_{00}$ term of eq. (10); the validity of this $v_{01}$ term has been checked previously [80] by the comparison with experiment of calculations of $(p, n)$ transitions to isobaric analog states. It is repulsive and has the form

$$
v_{01}^{\prime}(r)=-\left[4886 \frac{\mathrm{e}^{-4 r}}{4 r}-1176 \frac{\mathrm{e}^{-2.5 r}}{2.5 r}\right]+217 \delta(r)
$$

where, as before, the zero-range term is the pseudo-potential representing single nucleon exchange. (The value shown for this latter term is for a bombarding energy of $10 \mathrm{MeV}$ but again it only varies slowly with energy.) The magnitude of the volume integral of this term is almost exactly half that for the $(S, T)=(0,0)$ term,

$$
J_{01}^{\prime} / J_{00}^{\prime}=-0.50 \text {, }
$$

in agreement with the assumption often made [78, 79]. (The same assumption was used in extracting the empirical values given by eq. (20).) 
More sophisticated applications to nucleon scattering have been made recently $[5,6]$. These use a complex, energy- and density-dependent $G$-matrix for one nucleon in the continuum interacting with another bound in nuclear matter. Imaginary and spin-orbit parts of the optical potential are obtained at the same time as the real, central part. However, in keeping with our aim of a unified description of nucleon-nucleus and nucleus-nucleus scattering, it behooves us to examine further the adequacy for neutron and proton scattering of the interactions used here for heavy ion scattering. Our interactions are real, so that we only predict the real, central part of the nucleon optical potential; we treat the spin-orbit and imaginary terms phenomenologically. In principle, we could use the nucleon-nucleon spin-orbit effective interaction of Bertsch et al. [11] to generate the nucleon-nucleus spin-orbit coupling, but this was not done. (Our main purpose is to test the central interaction since it is that which is important for heavy ions.) Rather, for our present purpose, we simply took the imaginary and spin-orbit parts of the Becchetti-Greenlees global, phenomenological optical potential [78] and added them without change to our folded real potential. In general, this will not be exactly correct and will not yield the optimum fits to scattering data, but it does avoid some ambiguities of interpretation which could arise if these other parts of the potential were arbitrarily adjusted.

The folded real potentials of eq. (7b) were constructed using the same nuclear density distributions that were used for the heavy-ion calculations. Calculations were made for nucleon elastic scattering from ${ }^{40} \mathrm{Ca},{ }^{60} \mathrm{Ni}$ and ${ }^{208} \mathrm{~Pb}$, at energies of about 10 to $30 \mathrm{MeV}$, with generally similar results. Figure 6 shows some examples for ${ }^{60} \mathrm{Ni}$. In this figure, the folded potentials have been renormalized by a factor $N$ to optimize the fit to the data. The $N$ values, quoted in the figure, deviate from unity by negligible amounts $(\lesssim 5 \%)$. The agreement with experiment is quite satisfactory but not as good as can be obtained by simply using a phenomenological Woods-Saxon form for the real part of the potential. The fits could be improved by varying the parameters of the imaginary and spin-orbit terms and a certain amount of this could be justified. However, comparisons of the folded potential with the optimum Woods-Saxon real potential suggests that our theoretical potential has a mean square radius which is slightly too small. The same conclusion follows from comparison with phenomenological folding model analyses [79]. For example, these phenomenological potentials for ${ }^{60} \mathrm{Ni}+\mathrm{n}$ or $\mathrm{p}$ have $\left\langle r^{2}\right\rangle^{1 / 2} \approx 4.5 \mathrm{fm}$, while our folded potential has $\left\langle r^{2}\right\rangle^{1 / 2} \approx 4.1 \mathrm{fm}$, a difference of about $3 \mathrm{fm}^{2}$ in $\left\langle r^{2}\right\rangle$. The deficiency may be somewhat smaller for ${ }^{208} \mathrm{~Pb}+\mathrm{n}$ or $\mathrm{p}$; the folded potentials have $\left\langle r^{2}\right\rangle^{1 / 2} \approx 5.9 \mathrm{fm}$ (protons), $5.8 \mathrm{fm}$ (neutrons), while the phenomenological potentials have $\left\langle r^{2}\right\rangle^{1 / 2} \approx 6.0 \mathrm{fm}$.

The phenomenological folded potentials of Greenlees et al. [79] suffer from the same difficulty. It shows itself in their work either in the deduction of neutron distributions with unacceptably large radii (when their phenomenological interaction is constrained to have $\left\langle r^{2}\right\rangle=2.25 \mathrm{fm}^{2}$, not too different from the value $2.60 \mathrm{fm}^{2}$ for our M3Y interaction) or in the need for an interaction with a longer range, e.g. with $\left\langle r^{2}\right\rangle \approx 4.3 \mathrm{fm}^{2}$.

The cause of this discrepancy is not clear. It could be that the zero-range pseudo-potential of eq. (15) is not adequate to describe exchange, although it seems unlikely that this would explain the whole discrepancy. A large increase in the radii of the neutron density distributions would also improve the agreement although the ones we have used are consistent with other knowledge of neutron radii [3]. A density dependence of the interaction is capable of producing this kind of difference [81] although our calculations using the density-dependent DDD interaction (section 3.3.3) give almost the same results (but see [82]). Finally, the discrepancy observed might be due to neglect of contributions from the polarization potential $\Delta U$ of eq. (3) [40]. Whatever the explana- 

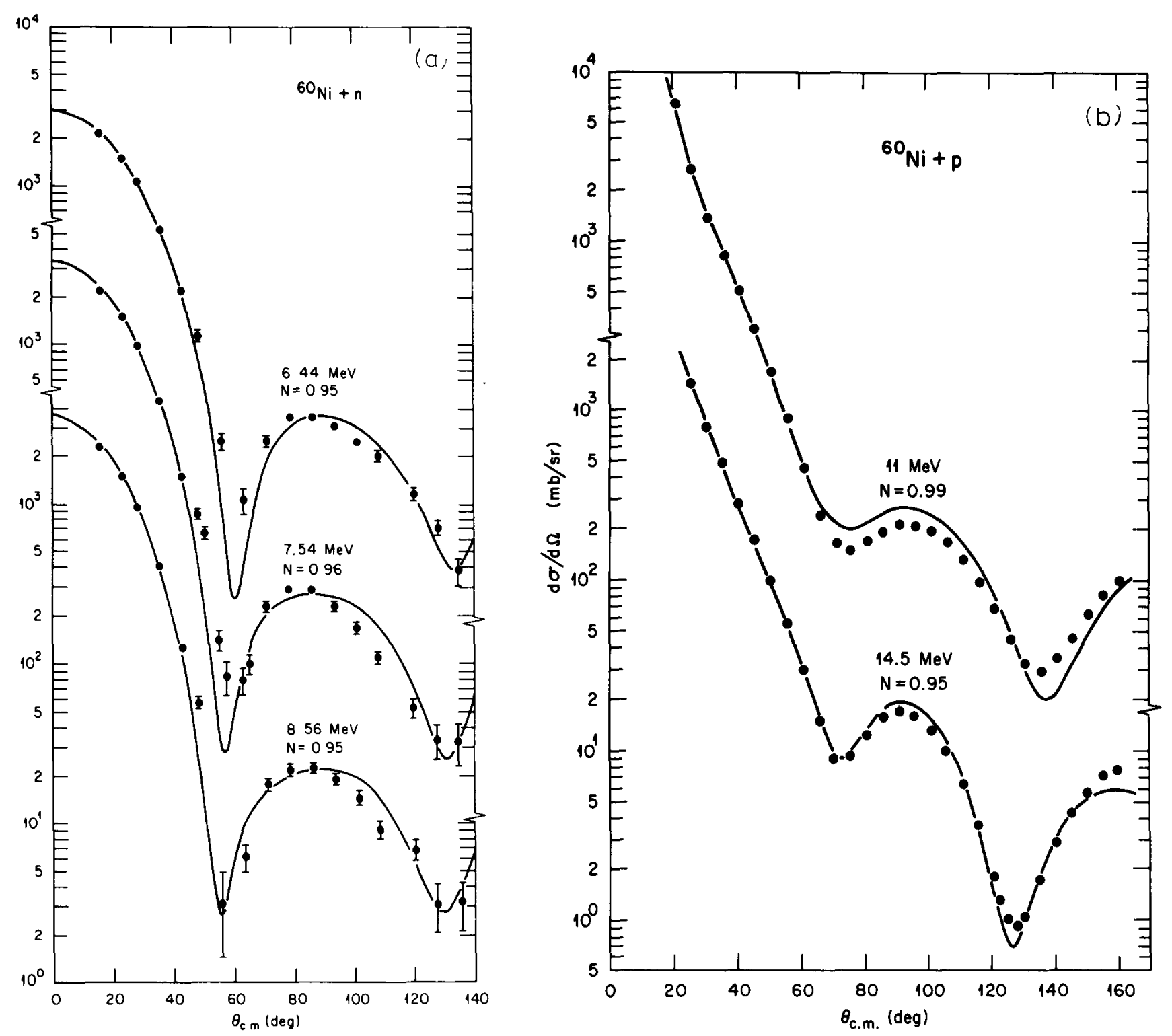

Fig. 6. The data for (a) neutrons and (b) protons scattering from ${ }^{60} \mathrm{Ni}$ compared with cross sections predicted using our folded real potential plus the spin-orbit and imaginary potentials of [78]. The folded real potentials were renormalized by the factors $N$ indicated.

tion, it must be remembered that any new feature introduced into the nucleon-nucleus problem might also affect the calculations of the heavy-ion potentials to be discussed later.

Applications of both the M3Y and the DDD interactions to inelastic nucleon scattering $[11,83]$ and $(p, n)$ transitions to analog states [80] have been described elsewhere. The agreement with experiment is good (except that the $v_{01}$ component of the DDD interaction seems'to be too weak [80]). Consequently, the interaction that we now apply to heavy-ion scattering gives a reasonably good account of nucleon scattering also, except for giving nucleon optical potentials with mean square radii that apparently are slightly too small. 


\section{Application to alpha scattering}

Before considering true heavy ions $(A>4)$, we discuss here two cases of alpha scattering which are of particular interest.

\subsection{The $\alpha+\alpha$ interaction}

It was shown recently [84] that the measured $\alpha+\alpha$ scattering phase shifts for $L=0-6$ could be accurately reproduced by a simple, local, real potential of Gaussian shape for center of-mass energies up to $20 \mathrm{MeV}$ and are approximately fitted for energies up to $40 \mathrm{MeV}$. This includes a good fit to the width and position of the $0^{+}$resonance at $92 \mathrm{keV}$ (i.e., the ${ }^{8} \mathrm{Be}$ ground state). Their potential is

$$
U(R)=-122.6225 \exp \left(-0.22 R^{2}\right) \mathrm{MeV} .
$$

We calculated the folded potential for the $\alpha+\alpha$ system using the M3Y interaction of eq. (10) and the zero-range approximation (15) for exchange. Two approaches were used for the density distribution of the alpha particle; both used a proton distribution $\rho_{\mathrm{p}}$ based upon electron scattering measurements [85] and assumed that $\rho_{\mathrm{n}}=\rho_{\mathrm{p}}$. In the first we took the model-independent charge density (table IV of [85]) for $r=0$ to $4.5 \mathrm{fm}$ (yielding an RMS charge radius of $1.66 \mathrm{fm}$ ) and unfolded the finite proton (and neutron) charge distribution using the prescription of section 3.4.1. This results in a depression in the center of the density distribution [85]. The second approach assumed a Gaussian form for the density distribution (hence with no central depression) with a range determined to give the "best" value [85] of the RMS charge radius of $1.67 \mathrm{fm}$. The corresponding alpha density is

$$
\rho_{\alpha}(r)=0.4229 \exp \left(-0.7024 r^{2}\right) \text {. }
$$

The resulting folded potentials are almost identical (so that the shape of the density distribution is not critical provided it is "reasonable" and has the correct RMS radius) and both are almost exactly the same as the phenomenological potential (23). This may be illustrated by quoting parameters for equivalent Gaussians, $-V_{0} \exp \left(-\gamma R^{2}\right)$, which have the same volume integral and RMS radius as the folded potentials and which are almost indistinguishable from them. The first density gives $V_{0}=121.3 \mathrm{MeV}, \gamma=0.2207 \mathrm{fm}^{-2}$, while the second gives $V_{0}=119.3 \mathrm{MeV}$, $\gamma=0.2183 \mathrm{fm}^{-2}$.

Consequently, the folded potential with the M3Y interaction provides a good fit to $\alpha+\alpha$ scattering in this energy range. This is an interesting system because it presents a favourable situation for the folded potential approach. The optical potential is real for center-of-mass energies below 17.3 MeV (where the ${ }^{7} \mathrm{Li}+\mathrm{p}$ channel opens). The tight binding of ${ }^{4} \mathrm{He}$ minimizes the polarization contributions $\Delta U$ to the potential. However, we have only included the effects of single nucleon exchange so that other exchange terms have been neglected; this requires further investigation.

\subsection{The scattering of $\alpha+{ }^{40} \mathrm{Ca}$}

This system is of interest because it exhibits [70] the so-called "anomalous large angle scattering". It is now understood [26] that this is a consequence of relatively weak absorption in this 
case which allows the scattering to be sensitive to the optical potential at separations $R$ that are smaller than usual. Then the scattering indicates [70] that the potential in the interior is deep and also that its shape deviates from the Woods-Saxon form.

As has been reported elsewhere [68], the folded potential based upon the M3Y interaction is very similar to the phenomenological potentials deduced [70] by fitting the scattering data. This folded potential gives fits to the data of comparable quality without the need for any significant renormalization. Very similar results are also obtained with the density-dependent DDD interaction. It has been noted that small changes in the effective interaction [68], or in the treatment of the exchange [57], would result in even better fits; however, the important feature for the present work is that the scattering data demand a potential like the folded one obtained with the M3Y interaction both in its shape and in the depth in the interior. This implies that the corrections from $\Delta U$ to the real potential are not large, even in the interior, in agreement with the estimates of Vinh Mau [23].

Nonetheless, it must be remarked that data for $\alpha+{ }^{40} \mathrm{Ca}$ at higher energies $(\gtrsim 100 \mathrm{MeV}$ ) rule out [69] a potential as deep as our folded one, even though they also support the use of a shape different from the Woods-Saxon one and similar to the folded shape. The folded potential is $\sim 260 \mathrm{MeV}$ deep at $R=0$, while the empirical ones are closer to $\sim 120 \mathrm{MeV}$. A suitable density dependence in the interaction will reduce the strength of the folded potential in the interior [86] to a value acceptable for the high-energy data; however, it remains to be seen whether such a modification can be made which will still fit the large-angle scattering at the lower energies as well as remaining compatible with both nucleon and heavy-ion scattering.

\section{Applications to heavy-ion scattering}

\subsection{Procedure}

The real folded potentials $U_{\mathrm{F}}(R)$ to be discussed here were (unless otherwise noted) constructed using the M3Y interaction of eq. (10) plus the zero-range exchange term (15) with $\hat{J}_{00}=-262 \mathrm{MeV}$ $\mathrm{fm}^{3}$ which are based upon the even-state Reid and odd-state Elliott forces. The density distributions described in section 3.4 were used.

When comparing with experimental data, the potential $U_{\mathbf{F}}(R)$ was fed into the optical model search code GENOA [87]. It was then multiplied by a renormalization factor $N$ which could be varied in order to optimize the fit to the data. Then the value $N \approx 1.0$ would indicate success for the model, while any systematic deviation of $N$ from unity would imply that corrections to the model were required. Some fluctuations of $N$ about unity can be expected because of idiosyncracies and uncertainties in the data and in the fitting procedures used and because of uncertainties in the densities used in calculating the potentials.

The criterion of fit is the usual one; we minimize the quantity

$$
\chi^{2}=\sum_{i}\left[\frac{\sigma_{\mathrm{ex}}\left(\theta_{i}\right)-\sigma_{\mathrm{th}}\left(\theta_{i}\right)}{\Delta \sigma_{\mathrm{ex}}\left(\theta_{i}\right)}\right]^{2},
$$

where $\sigma_{\mathrm{th}}\left(\theta_{i}\right)$ are the calculated differential cross sections, $\sigma_{\mathrm{ex}}\left(\theta_{i}\right)$ are the measured ones and $\Delta \sigma_{\mathrm{ex}}\left(\theta_{i}\right)$ are the uncertainties ("errors") associated with them.

In some cases at least, it might be argued that we should do coupled-channels calculations 


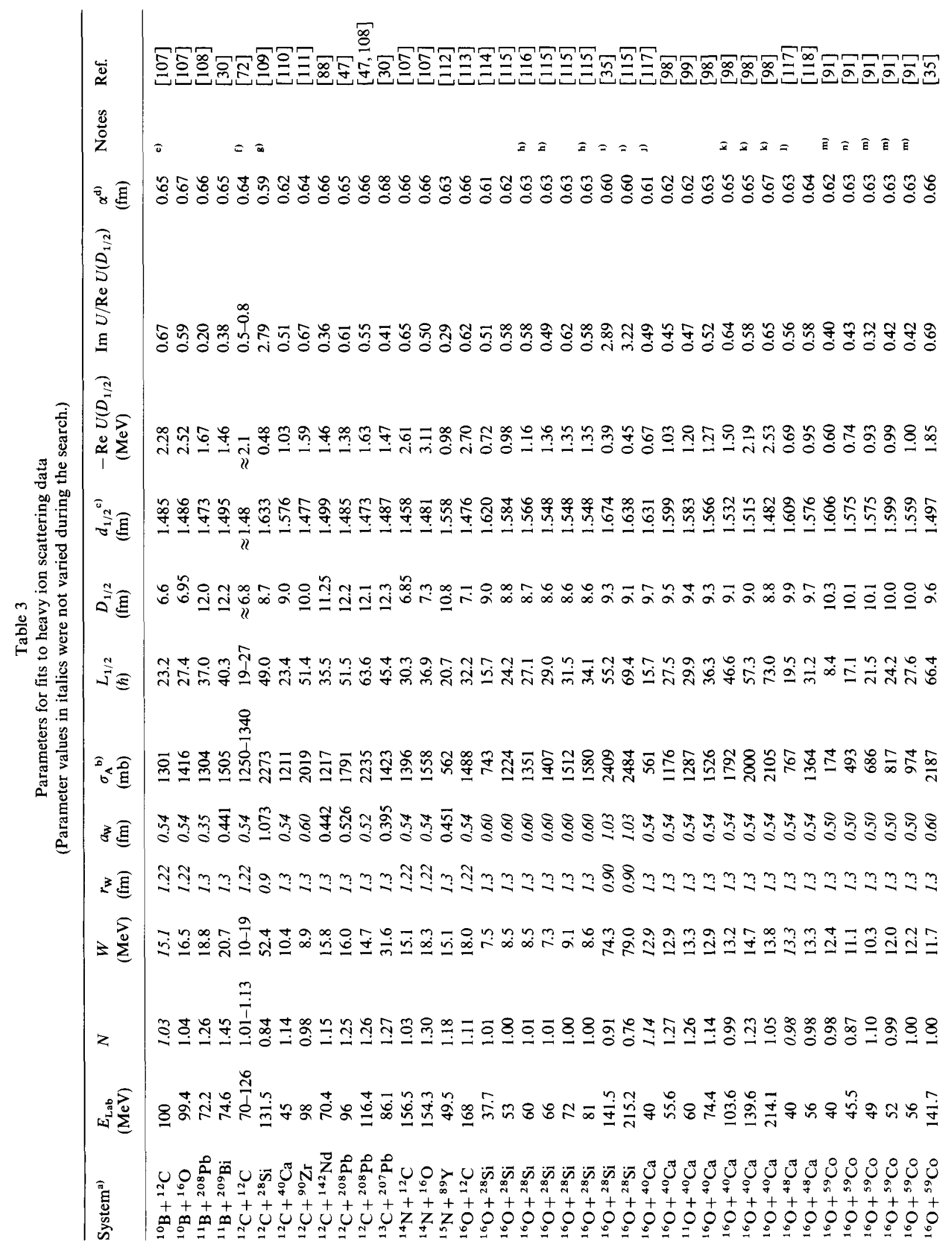




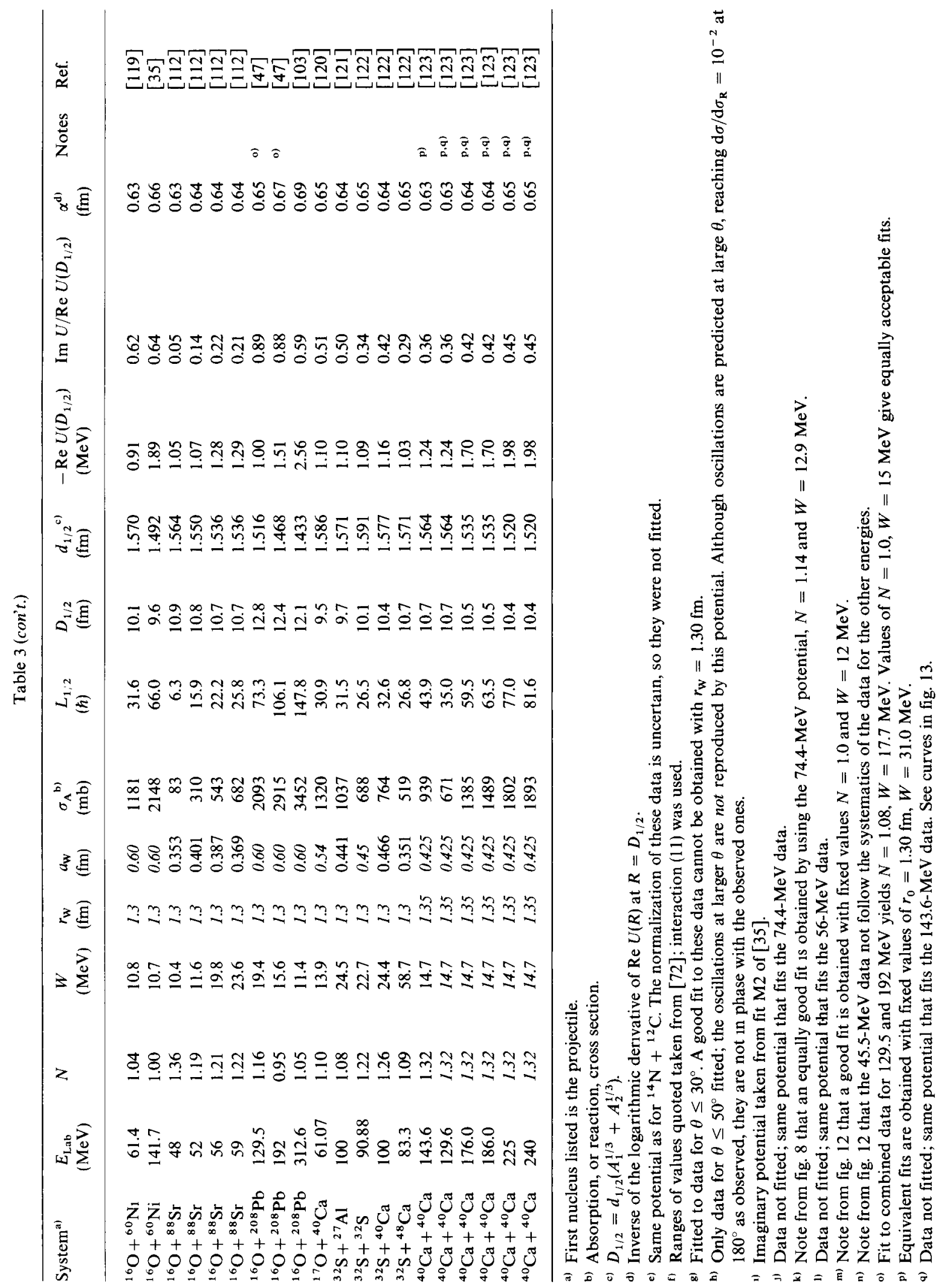


rather than use the one-channel optical model approach. Some of the nuclei studied here, such as

${ }^{12} \mathrm{C}$ and ${ }^{28} \mathrm{Si}$, exhibit large inelastic cross sections for exciting low-lying $2^{+}$states. (We have deliberately avoided other systems such as ${ }^{12} \mathrm{C}+{ }^{150} \mathrm{Nd}$ for which coupled channels are essential [88]; even in that case, however, the effect on the real potential did not seem to be large.) However, exploratory calculations with ${ }^{12} \mathrm{C}+{ }^{12} \mathrm{C}$ and with ${ }^{16} \mathrm{O}+{ }^{28} \mathrm{Si}$ indicated that while coupling effects need not be negligible, especially upon the imaginary potential, they would not qualitatively change any of our results. This conclusion is reinforced by some other recent coupled channel calculations for ${ }^{16} \mathrm{O}+{ }^{28} \mathrm{Si}$ [89] (see also P.D. Bond in [15]). In this case, explicitly including the coupling led to a reduction of 10 to $15 \%$ in the real potential required, while the work on ${ }^{12} \mathrm{C}+{ }^{12} \mathrm{C}[72]$ suggested the opposite effect. Of course, the coupling effects observed will depend upon the type of optical potential used as well as the system being studied. Another reason for believing that our results are not strongly affected by these couplings is that the renormalizing factors $N$ which are obtained by optimizing the fits to data (table 3) do not show any obvious correlation with the "collectivity" of the nuclei involved.

\subsection{The imaginary potential}

Since the effective interaction used here is real, the imaginary, absorptive, part of the optical potential has to be treated phenomenologically. The simplest assumption is that it has the same shape as the real folded potential so that only its strength is an adjustable parameter. Indeed, this is found to be sufficient for good fits to scattering data in a number of cases. However, for other cases it is found that significantly better fits can be obtained if a Woods-Saxon shape is used; when the scattering is determined by peripheral collisions, this indicates a need for real and imaginary potentials with different slopes in the vicinity of the strong absorption radius. Finally, there are a few cases (e.g. ${ }^{12} \mathrm{C}+{ }^{12} \mathrm{C}$ and ${ }^{16} \mathrm{O}+{ }^{28} \mathrm{Si}$ ) where acceptable fits cannot be obtained at all with real and imaginary potentials of the same shape.

A Woods-Saxon form

$$
\operatorname{Im} U(R)=-W\left(\mathrm{e}^{x}+1\right)^{-1}, \quad x=\left(R-R_{\mathrm{W}}\right) / a_{\mathrm{W}}
$$

is characterized by three parameters, $W, R_{\mathrm{W}}$ and $a_{\mathrm{W}}$, and this is one too many to be determined unambiguously in most cases. Consequently, whenever possible, we chose to fix the imaginary potential radius parameter $r_{\mathrm{W}}$, where $R_{\mathrm{W}}=r_{\mathrm{w}}\left(A_{1}^{1 / 3}+A_{2}^{1 / 3}\right)$. In almost all cases, the value $r_{\mathrm{w}}=1.30 \mathrm{fm}$ was found to be satisfactory. Exceptions occurred when fitting some data for ${ }^{12} \mathrm{C}+{ }^{28} \mathrm{Si}$ and ${ }^{16} \mathrm{O}+{ }^{28} \mathrm{Si}$, for which a smaller value of $r_{\mathrm{W}} \approx 0.90 \mathrm{fm}$ seemed to be required. The value of $r_{\mathrm{W}}=1.22 \mathrm{fm}$ was adopted for the lightest systems simply to be consistent with the analyses of ${ }^{12} \mathrm{C}+{ }^{12} \mathrm{C}$ scattering reported elsewhere [72].

The surface diffuseness parameter was initially set at $a_{\mathrm{w}}=0.60 \mathrm{fm}$ and left at that value unless the fit to the data demanded a change. (Again, $a_{\mathrm{w}}=0.54 \mathrm{fm}$ was used for the lightest systems for comparison with the earlier ${ }^{12} \mathrm{C}+{ }^{12} \mathrm{C}$ analyses [72].) In all cases where a change was required (except for the "anomalous" ${ }^{12} \mathrm{C},{ }^{16} \mathrm{O}+{ }^{28} \mathrm{Si}$ cases just mentioned) it was in the direction of reducing $a_{\mathrm{w}}$, that is of making the imaginary potential steeper in the surface. 


\subsection{Previous results}

The M3Y interaction (10), together with the zero-range exchange term (15), has previously been applied [90] to ${ }^{16} \mathrm{O}+{ }^{63,65} \mathrm{Cu}$ scattering at energies of 2.5 to $2.9 \mathrm{MeV}$ per nucleon. The density distributions used appear to be similar to those we use here, but the exchange pseudo-potential is somewhat stronger, $\hat{J}_{00}=409 \mathrm{MeV} \mathrm{fm}^{3}$. This would result in a potential at the strong absorption radius about $10 \%$ larger than the value we use. These authors find good agreement with the data, with optimum $N$ values differing from unity by only a few per cent.

The scattering of ${ }^{16} \mathrm{O}+{ }^{59} \mathrm{Co}$ at 2.25 to $3.5 \mathrm{MeV}$ per nucleon has also been fitted [91] in a similar way with similar results. We have re-analyzed these data and will return to them later.

In other studies, this M3Y interaction has been used to estimate the quadrupole scattering for ${ }^{10,11} \mathrm{~B}$ and ${ }^{12} \mathrm{C}$ on ${ }^{27} \mathrm{Al}$ and hence to explain differences seen in the elastic scattering at large angles [92]. It has also been used to estimate the vector spin-orbit coupling for various heavy ions [93].

The extensive analyses of ${ }^{12} \mathrm{C}+{ }^{12} \mathrm{C}$ scattering from 5 to $10 \mathrm{MeV}$ per nucleon already published [72] used the interaction (11) instead of (10), i.e. with only the OPEP in odd-states. This produces a potential $3 \%$ deeper at $R=0$ but slightly less deep for $R>6.5 \mathrm{fm}$. Although in this case the scattering near $90^{\circ}$ is sensitive even to these small changes, the overall effect on the optimum $N$ values deduced is small (at most, a few per cent). Consequently, we did not reanalyze these data with the other interaction (10).

Previously published analyses of ${ }^{16} \mathrm{O}+{ }^{28} \mathrm{Si}[35,94]$ and ${ }^{16} \mathrm{O}+{ }^{59} \mathrm{Co},{ }^{60} \mathrm{Ni}[35,48]$ were also made with the interaction (11) and in addition the single nucleon exchange contribution (15) was neglected. Further, density distributions were used which were slightly different from those used here, so, for consistency, these data have been reanalyzed.

\subsection{Present results}

The results of fitting data for some 30 different systems, often at more than one energy, are summarized in table 3. Some examples of the fits are shown in figs. 7-18. (Additional results for ${ }^{6,7} \mathrm{Li}$ and ${ }^{9} \mathrm{Be}$ scattering are discussed later.) The sources of the data are indicated in the table. The particular sets of data used were chosen (mainly) because numerical values of the cross sections were available. (It would have been particularly interesting to have been able to study some heavier systems, such as $\mathrm{Kr}+\mathrm{Bi}$ or $\mathrm{Xe}+\mathrm{Pb}$, but because of experimental difficulties, there are no data available in which the elastic scattering has been fully resolved from nonelastic scattering. Another problem with such heavy systems is that the long-range absorption associated with Coulomb excitation makes the scattering less sensitive to the real potential [24].)

In most cases the scattering only determines the potential in the vicinity of the strong absorption radius (see, for example $[8,90]$ ). As has been stressed before $[47,95]$, when the angular distribution resembles that of Fresnel diffraction, the magnitude of the oscillations about the Rutherford cross section before the onset of the "shadow" are especially important in determining the strengths of both the real and the imaginary potential near the strong absorption radius. (Contrary to statements by adherents of either the "Fresnel" or the "rainbow" interpretation, the characteristics of the scattering are not determined in general by either the imaginary potential alone or the real potential alone; both are required for good fits to the data.) Combined with the rate of fall of the cross section in the shadow region, the data also put constraints upon the slope of the imaginary potential. (The slope of the real potential is, of course, determined by the folding procedure.) 


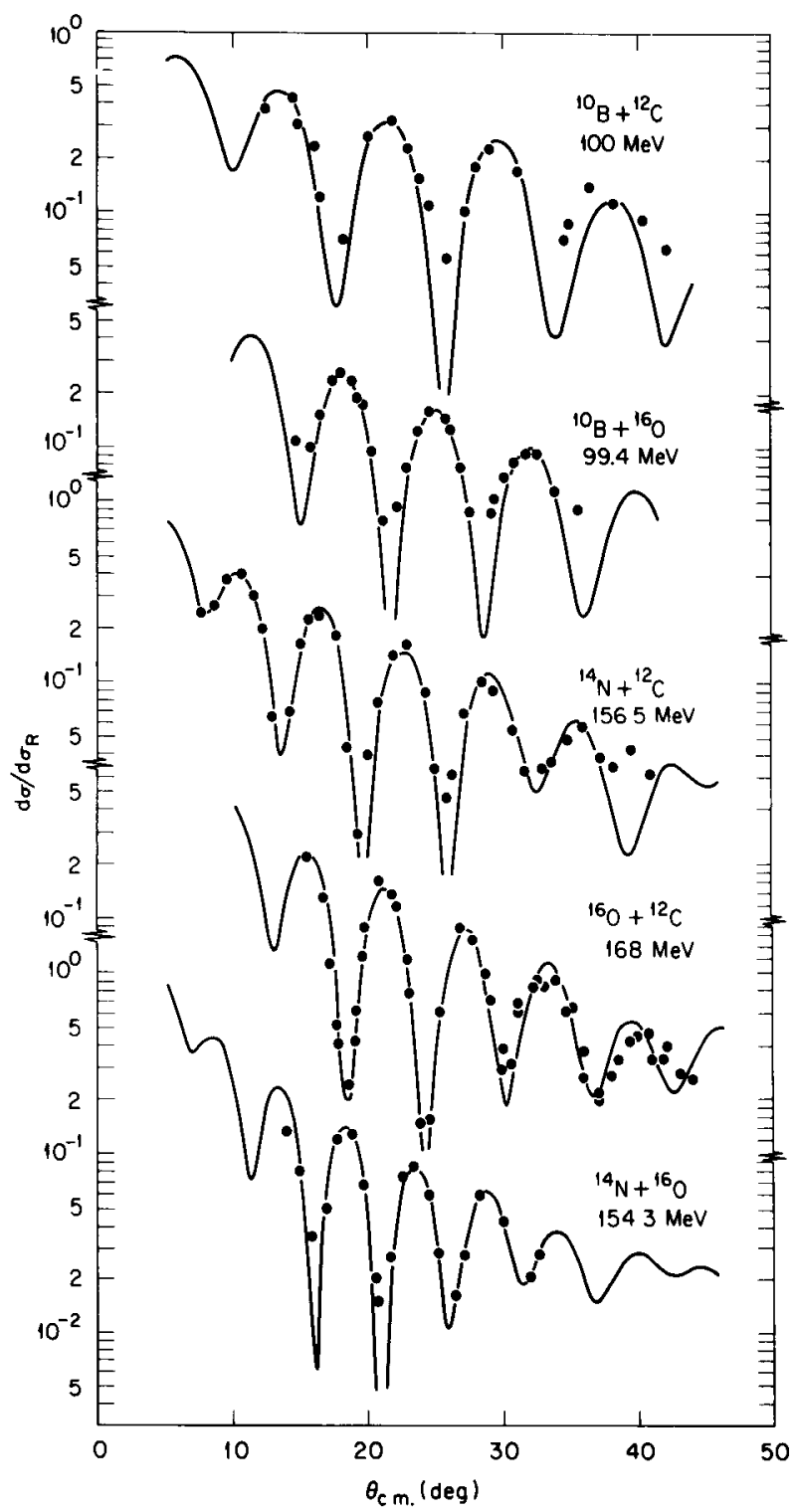

Fig. 7. Comparison between predicted and measured cross sections for some light heavy-ion systems. The real potential normalizations and imaginary potential parameters are given in table 3.

Table 3 summarizes the results, giving the optimum values of the real, folded potential normalization factors $N$, as well as the values of the parameters $W, r_{\mathrm{W}}, a_{\mathrm{W}}$ for the imaginary potential. Also listed in each case are the absorption (reaction) cross section $\sigma_{\mathrm{A}}$, the angular momentum $L_{1 / 2}$ for which the transmission coefficient $T_{L_{1} / 2}=\frac{1}{2}$ (obtained by linear interpolation between the values for integral $L$ ) and the distance of closest approach $D_{1 / 2}$ for the Rutherford orbit with the angular momentum $L_{1 / 2}$,

$$
D_{1 / 2}=\frac{1}{k}\left[n+\left(n^{2}+L_{1 / 2}^{2}\right)^{1 / 2}\right]
$$

where $n$ is the usual Sommerfeld parameter and $k$ is the wave number. We use this distance $D_{1 / 2}$ 
as our measure of the strong absorption radius. Table 3 includes the value of the real potential at $R=D_{1 / 2}$, the ratio of imaginary to real potential at that radius and $\alpha$, the inverse of the logarithmic derivative of the real part there,

$$
\alpha=-U\left(D_{1 / 2}\right)(\mathrm{d} U(R) / \mathrm{d} R)_{R=D_{1 / 2}}^{-1},
$$

as an indicator of the slope of the real potential. (If the real potential were truly exponential near $R=D_{1 / 2}$, it would have the form $\sim \exp (-R / \alpha)$.)

\subsubsection{The real potential normalization}

The mean and RMS deviation of the $N$ values in table 3 is

$$
N \approx 1.11 \pm 0.13
$$

indicating that on the average the folding model with the M3Y interaction predicts the heavy ion real potential quite accurately. Indeed, even the deviation of $+10 \%$ of the mean $N$ from unity could be due to an underestimate of the exchange contribution when using the zero-range approximation (15), as we have already anticipated. Only five cases have $N$ values differing from the mean by $20 \%$ or more. Two of these $\left({ }^{16} \mathrm{O}+{ }^{88} \mathrm{Sr}\right.$ at $48 \mathrm{MeV}$ and ${ }^{16} \mathrm{O}+{ }^{59} \mathrm{Co}$ at $\left.45.5 \mathrm{MeV}\right)$ appear anomalous in the sense that results for the same systems at other energies do not show as large deviations. The largest deviation occurs for ${ }^{11} \mathrm{~B}+{ }^{209} \mathrm{Bi}$ with $N=1.45$ and might indicate some error in the density distribution we have used for ${ }^{11} \mathrm{~B}$. We assumed ${ }^{11} \mathrm{~B}$ to be the same as ${ }^{12} \mathrm{C}$ except for a proton hole in the $1 \mathrm{p}_{3 / 2}$ shell. If, instead, we take the $1 \mathrm{p}_{3 / 2}$ binding energies in ${ }^{11} \mathrm{~B}$ to be equal to the observed separation energies for a neutron or a proton from ${ }^{11} \mathrm{~B}$, then the RMS radius of ${ }^{11} \mathrm{~B}$ increases from 2.28 to $2.47 \mathrm{fm}$, and the folded potential for ${ }^{11} \mathrm{~B}+{ }^{209} \mathrm{Bi}$ increases by $40 \%$ at the strong absorption radius. This procedure implies too large a charge radius $(2.55 \mathrm{fm})$ for ${ }^{11} \mathrm{~B}[59,96]$, but it does emphasize again the sensitivity of the potential to the density assumed.

More detailed discussion of some specific cases is given below.

\subsubsection{Energy dependence}

We find no compelling need for any dependence on bombarding energy of either the real or the imaginary potential (with the exception of the imaginary potentials for ${ }^{12} \mathrm{C}+{ }^{12} \mathrm{C}$ [72] and for the case ${ }^{16} \mathrm{O}+{ }^{28} \mathrm{Si}$ [94] to be discussed later). Figures $8,13,15,17$ and 18 show various sets of data which span ranges of energy yet which are fitted well in each case by a single, energy-independent potential. That is not to say that the data are not compatible with a weak energy dependence. Table 3 shows that the optimum $N$ values fluctuate somewhat with energy; the fluctuations appear to be "random", but they do indicate that the data could accommodate a slow variation of $N$ with energy. Indeed, the earlier analyses of ${ }^{12} \mathrm{C}+{ }^{12} \mathrm{C}$ [72] did seem to suggest a slow increase of $N$ with energy.

However, it should be remembered that we are not considering a very wide range of energy per nucleon; the largest range is for ${ }^{16} \mathrm{O}+{ }^{40} \mathrm{Ca}$ where $E / A \simeq 3.5$ to $13.4 \mathrm{MeV}$. For example, arguments [97] based upon empirical nucleon-nucleus potentials would suggest a reduction in the real potential strength of only about $6 \%$ in this energy range.

\subsubsection{Light systems}

The light systems illustrated in fig. 7 exhibit angular distributions with diffraction-like oscillations. Only a limited angular range is covered and, in some cases, the absolute normalizations of 


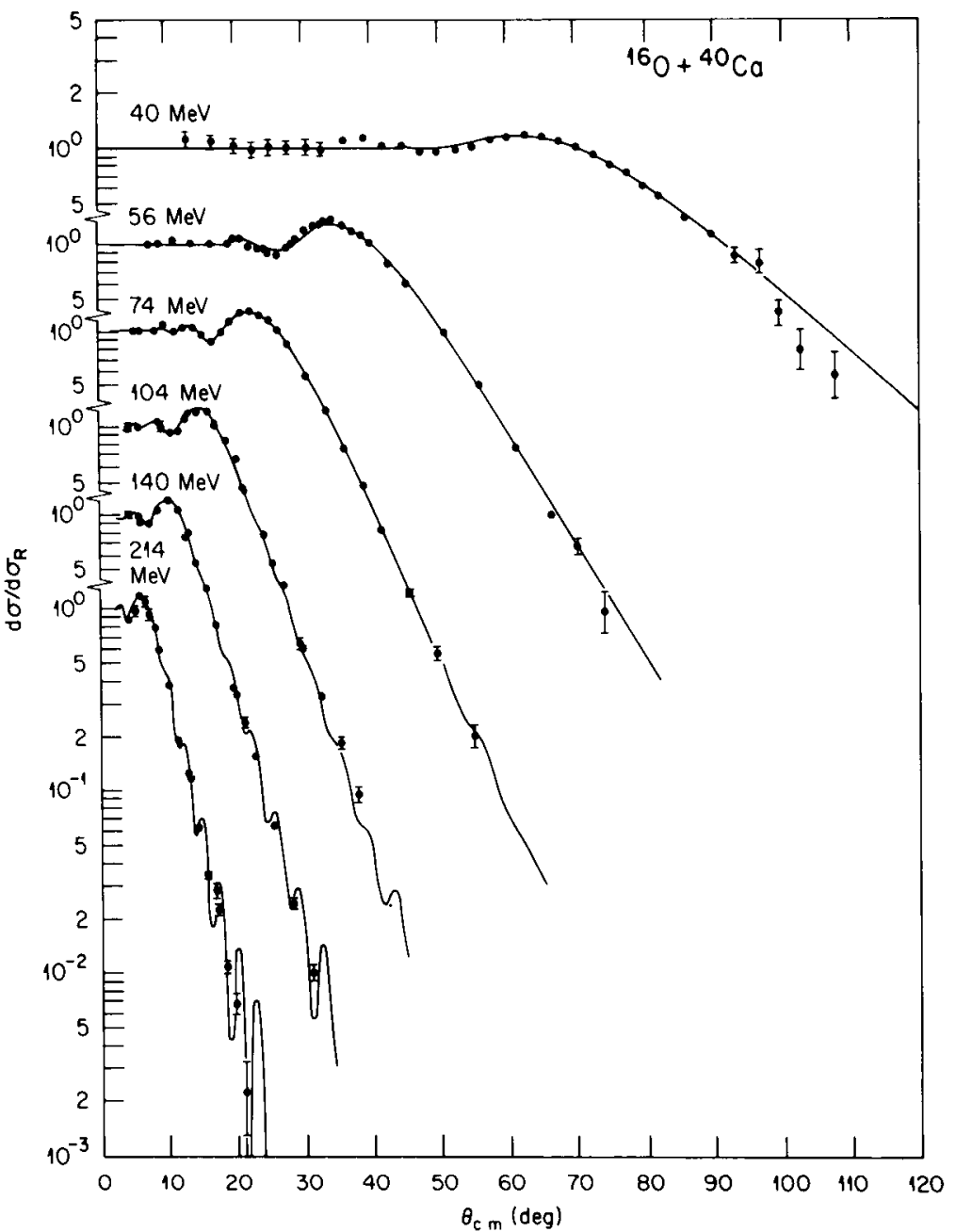

Fig. 8. The scattering of ${ }^{16} \mathrm{O}+{ }^{40} \mathrm{Ca}$ The curves are predictions using the potential (table 3) fitted to the data at $74 \mathrm{MeV}$.

the data are not precisely determined. Fortunately, this uncertainty mainly affects the strength of $\operatorname{Im} U$, while $\operatorname{Re} U$ is determined primarily by the position of the diffraction peaks; nonetheless there remains some uncertainty in the values of $N$. In particular, the data for ${ }^{10} \mathrm{~B}+{ }^{12} \mathrm{C}$ were not fitted; the curve shown is for the same potential parameters as for ${ }^{14} \mathrm{~N}+{ }^{12} \mathrm{C}$.

The ${ }^{12} \mathrm{C}+{ }^{12} \mathrm{C}$ scattering at $70-126 \mathrm{MeV}$ was discussed earlier [72]. The interesting but notoriously difficult cases of ${ }^{12} \mathrm{C}+{ }^{12} \mathrm{C}$ at lower energies and of ${ }^{16} \mathrm{O}+{ }^{16} \mathrm{O}$ have not yet been studied carefully.

\subsubsection{Scattering of ${ }^{16,17} \mathrm{O}+{ }^{40,48} \mathrm{Ca}$}

Figure 8 shows that the potential which gives the optimum fit to the ${ }^{16} \mathrm{O}+{ }^{40} \mathrm{Ca}$ data for $74.4 \mathrm{MeV}$ also gives good fits to the data at other energies. The optimum (minimum $\chi^{2}$ ) parameter values (table 3) for these other energies do not result in significantly better fits except at $55.6 \mathrm{MeV}$ where the oscillations about the Rutherford cross section are better reproduced by increasing $N$. This latter point is emphasized by the linear plot in fig. 9 where we see that the value $N=1.14$, which is optimum at $74.4 \mathrm{MeV}$ and sufficient at the other energies, predicts too small amplitude 


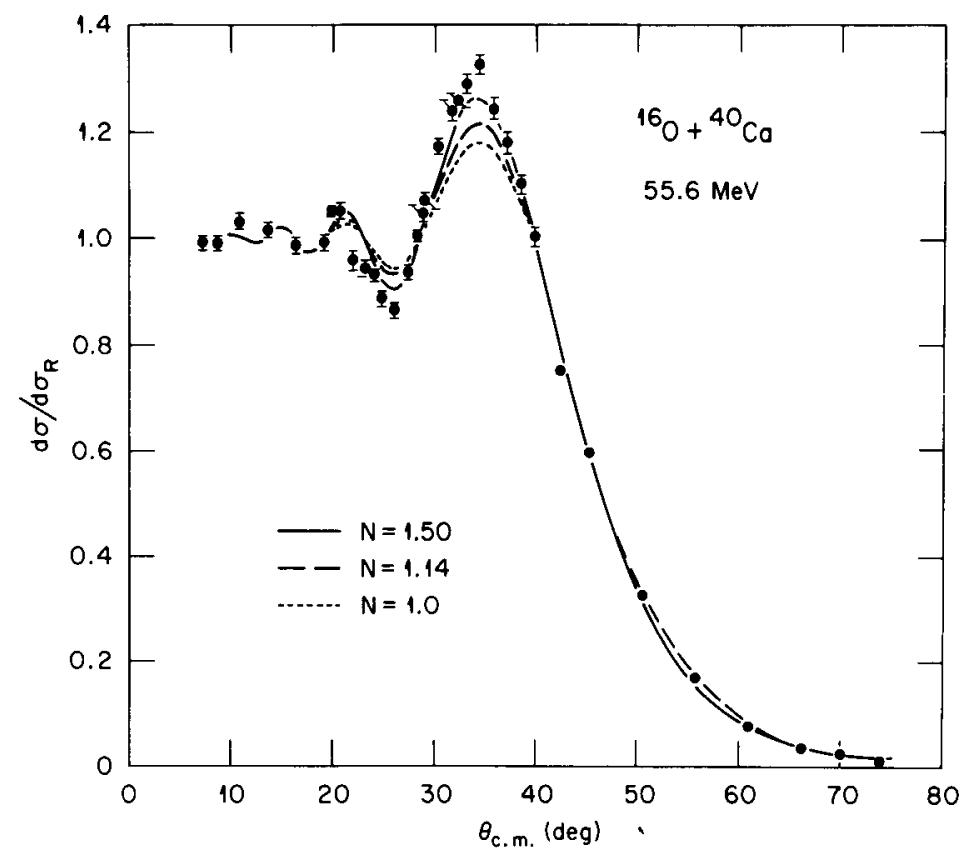

Fig. 9. The scattering predicted for ${ }^{16} \mathrm{O}+{ }^{40} \mathrm{Ca}$ at $55.6 \mathrm{MeV}$ for various normalizations $N$ of the folded, real potential. In each case, the imaginary potential parameters were varied to optimize the fit to the data. $N=1.50$ is the optimum value when the imaginary diffuseness is also allowed to vary (see text), while $N=1.14$ is the optimum value for the data at $74 \mathrm{MeV}$ (fig. 8).

for these oscillations. Indeed, these oscillations alone would imply $N>1.5$. Minimum $\chi^{2}$ is obtained with $N=1.27$ if we constrain $a_{\mathrm{W}}=0.54 \mathrm{fm}$ (table 3) giving a peak with $\mathrm{d} \sigma / \mathrm{d} \sigma_{\mathrm{R}}=1.23$. Allowing $a_{\mathrm{W}}$ to vary as well results in $N=1.50, W=27.8 \mathrm{MeV}, a_{\mathrm{W}}=0.396 \mathrm{fm}$ and a peak $\mathrm{d} \sigma / \mathrm{d} \sigma_{\mathbf{R}}=1.27$ (the solid curve in fig. 9).

The cross sections at 55.6 MeV have been remeasured [98] and the results shown here have been confirmed. Further, data are also available at $60 \mathrm{MeV}$ [99], and fits to these show characteristics similar to those at $55.6 \mathrm{MeV}$, so that perhaps something is changing for the ${ }^{16} \mathrm{O}+{ }^{40} \mathrm{Ca}$ system as the bombarding energy falls below $70 \mathrm{MeV}$. Meanwhile, measurements from $20^{\circ}$ to $180^{\circ}$ at $50 \mathrm{MeV}$ have revealed an "anomalous large-angle scattering" which indicates a reduction in the absorption at this energy, at least in the surface [100]. The potential that fits the 74.4-MeV data (table 3 and fig. 9) gives cross sections at large angles at $50 \mathrm{MeV}$ that are from one to two orders of magnitude too small. However, simply reducing the strength of $W$ from 12.9 to $7.5 \mathrm{MeV}$ does produce cross sections with qualitatively the correct behaviour although it is far from a good fit to the data. Clearly, other readjustments are necessary. A similar reduction in $W$ also improves the fit to the peak cross section at $55.6 \mathrm{MeV}$ even with $N=1.14$ but again does not provide an optimum fit to the data overall. If a greater transparency at these energies is the correct explanation, then this may provide another situation in which we can test the potential at distances inside the strong absorption radius.

Figure 10 shows that the potential (table 3) that fits the $56-\mathrm{MeV}$ data for ${ }^{16} \mathrm{O}+{ }^{48} \mathrm{Ca}$ also fits the $40-\mathrm{MeV}$ data. This potential has $N=0.98$, compared to 1.14 for ${ }^{16} \mathrm{O}+{ }^{40} \mathrm{Ca}$. This may be due to Negele's ${ }^{48} \mathrm{Ca}$ density being a little too large; we have a ${ }^{48} \mathrm{Ca}-{ }^{40} \mathrm{Ca}$ RMS radius difference of $0.2 \mathrm{fm}$ compared to a value $0.1 \mathrm{fm}$ recently deduced [65] from $1 \mathrm{GeV}$ proton scattering.

Figure 11 shows the cross sections predicted at $177^{\circ}$ near the Coulomb barrier using the poten- 


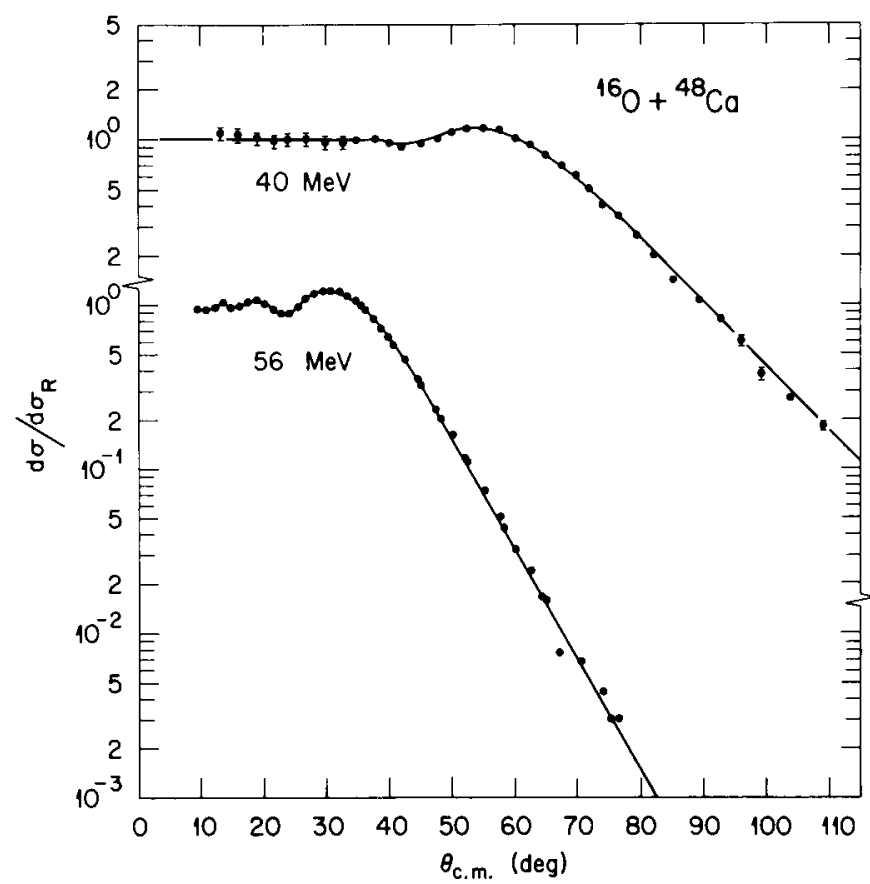

Fig. 10. Comparison with data for ${ }^{16} \mathrm{O}+{ }^{48} \mathrm{Ca}$ at 40 and $56 \mathrm{MeV}$ using the potential of table 3 .

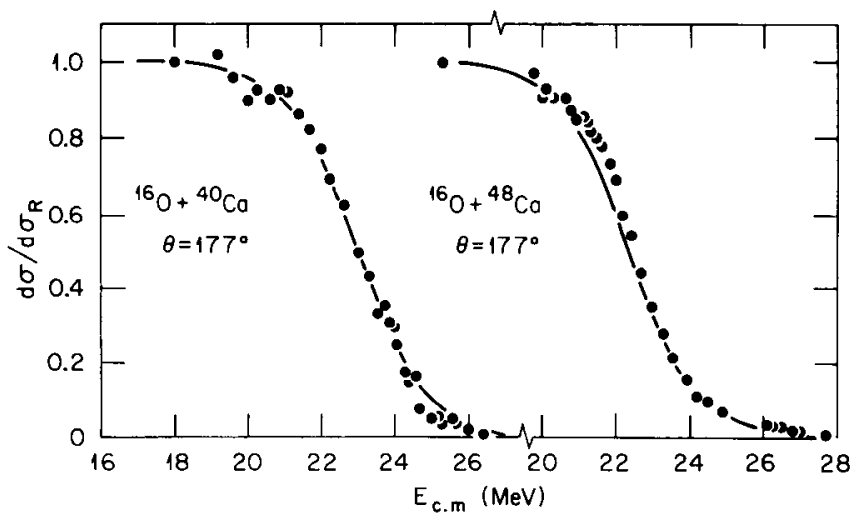

Fig. 11. Excitation functions for back-scattering of ${ }^{16} \mathrm{O}+{ }^{40,48} \mathrm{Ca}$. The curves are for $N=1.14\left({ }^{40} \mathrm{Ca}\right), 1.0\left({ }^{48} \mathrm{Ca}\right), W=13 \mathrm{MeV}$, $r_{\mathrm{w}}=1.3 \mathrm{fm}$ and $a_{\mathrm{w}}=0.54 \mathrm{fm}$.

tials which fit the scattering data at higher energies $\left(74.4 \mathrm{MeV}\right.$ for ${ }^{40} \mathrm{Ca}$, $56 \mathrm{MeV}$ for ${ }^{48} \mathrm{Ca}$; table 3 ); there is reasonable agreement with the measurements [101]. (These energies are below those at which the "anomalies" for ${ }^{40} \mathrm{Ca}$ are observed [100].)

There is also one measurement for ${ }^{17} \mathrm{O}+{ }^{40} \mathrm{Ca}$. The ${ }^{17} \mathrm{O}$ density was taken to be the same as the shell model density for ${ }^{16} \mathrm{O}$ with the addition of a $1 \mathrm{~d}_{5 / 2}$ neutron bound in the same shell model potential. This gives a folded potential which is $24 \%$ larger for ${ }^{17} \mathrm{O}$ near the strong absorption radius of $9.5 \mathrm{fm}$. The optimum fit to the data (table 3) yields $N=1.10$, very close to the value 1.14 for ${ }^{16} \mathrm{O}$ on the same target. It is encouraging that this isotope dependence is so closely reproduced. 


\subsubsection{Scattering of ${ }^{16} \mathrm{O}+{ }^{59} \mathrm{Co},{ }^{60} \mathrm{Ni}$}

The analysis of ${ }^{16} \mathrm{O}+{ }^{59} \mathrm{Co}$ and ${ }^{60} \mathrm{Ni}$ at $142 \mathrm{MeV}$ with folded potentials has been discussed in detail elsewhere [35]. The analysis was repeated with the improved densities described in section 3.4 , with the interaction (10), and with the imaginary radius parameter $r_{\mathrm{w}}=1.30 \mathrm{fm}$ adopted here as standard. The fits to the data are indistinguishable from those shown before [35] and optimum $N$ values of unity are obtained (table 3 ). The earlier work also showed that the potential which fit at $142 \mathrm{MeV}$ also fitted data at $61 \mathrm{MeV}$ and for various energies between 40 and $56 \mathrm{MeV}$ [35]. The same remains true for the present, slightly revised potential; the optimum value is $N=1.04$ at $61 \mathrm{MeV}$ but 1.00 gives essentially the same results. (The optimum real potential values at the strong absorption radii are included in fig. 2.)

Data for ${ }^{16} \mathrm{O}+{ }^{59} \mathrm{Co}$ are now also available [91] for energies from 40 to $56 \mathrm{MeV}$. Contrary to the case for ${ }^{60} \mathrm{Ni}$, it was found necessary to reduce the value of $a_{\mathrm{w}}$ to about $0.50 \mathrm{fm}$ in order to fit these data. A simple variation of $W$ is not sufficient; $a_{\mathrm{W}}=0.60 \mathrm{fm}$ causes the theoretical cross sections to fall off too fast. Fluctuations in the best-fit parameter values were obtained similar to those found by the original authors [91] (who used a similar folded potential). These fluctuations were reduced when $a_{\mathrm{w}}$ was frozen at the value $0.50 \mathrm{fm}$ (table 3). Indeed, fig. 12 shows that very good fits can be obtained with a single potential in which $N=1.0$ and $W=12 \mathrm{MeV}$ were fixed, except at the one bombarding energy of $45.5 \mathrm{MeV}$. The dashed curve in fig. 12 shows that a good fit at this energy requires a reduction of both $N$ to 0.87 and $W$ to $11.1 \mathrm{MeV}$ (table 3). The origin of this discrepancy is not understood.

\subsubsection{The ${ }^{12} \mathrm{C}+{ }^{142} \mathrm{Nd}$ system}

This case is of interest because it illustrates the sensitivity to the neutron distribution assumed. The Batty-Greenlees [64] shell model potential we have adopted (section 3.4) with $V=60 \mathrm{MeV}$ for the protons gives a charge radius for ${ }^{142} \mathrm{Nd}$ of $\left\langle r^{2}\right\rangle^{1 / 2}=4.93 \mathrm{fm}$, in agreement with electron scattering [59]. (A configuration of $\left(\mathrm{gg}_{7 / 2}\right)^{8}\left(2 \mathrm{~d}_{5 / 2}\right)^{2}$ outside the closed $Z=50$ shell was assumed.) Further, a binding energy of $7.3 \mathrm{MeV}$ is obtained for the $2 \mathrm{~d}_{5 / 2}$ proton, agreeing with the observed proton separation energy. At first it was thought that $V=50 \mathrm{MeV}$ and the same geometry would be a reasonable choice for the neutron potential. This gave a neutron RMS radius which exceeded the proton one by $0.14 \mathrm{fm}$, again apparently reasonable. However, the resulting folded potential required a renormalization factor of $N=1.39$ in order to fit the scattering data [88], and especially to match the large magnitude of the oscillations about the Rutherford value. Further, the choice $V=50 \mathrm{MeV}$ leads to a binding of $13.1 \mathrm{MeV}$ for the least-bound neutrons, while the observed separation energy is $9.8 \mathrm{MeV}$.

Reducing the neutron well-depth to $V=45 \mathrm{MeV}$ gives the appropriate binding for the leastbound neutron and an RMS radius $0.26 \mathrm{fm}$ larger than that for the protons. Then the optimum $N$ value for the folded potential (table 3 ) is 1.15 , or $20 \%$ smaller and now acceptably close to the average value. (The fit to the data is indistinguishable from that shown in [88].) It now becomes of considerable interest to know whether such a large neutron-proton radius difference can be confirmed independently, perhaps by high-energy proton scattering, and thus test the usefulness of heavy-ion scattering as a probe of such differences.

\subsubsection{Some more massive projectiles}

As noted earlier, data for very heavy systems are not available. We do have ${ }^{40} \mathrm{Ca}+{ }^{40} \mathrm{Ca}$ data for several energies; fig. 13 shows the Orsay data compared with folding model predictions. The 


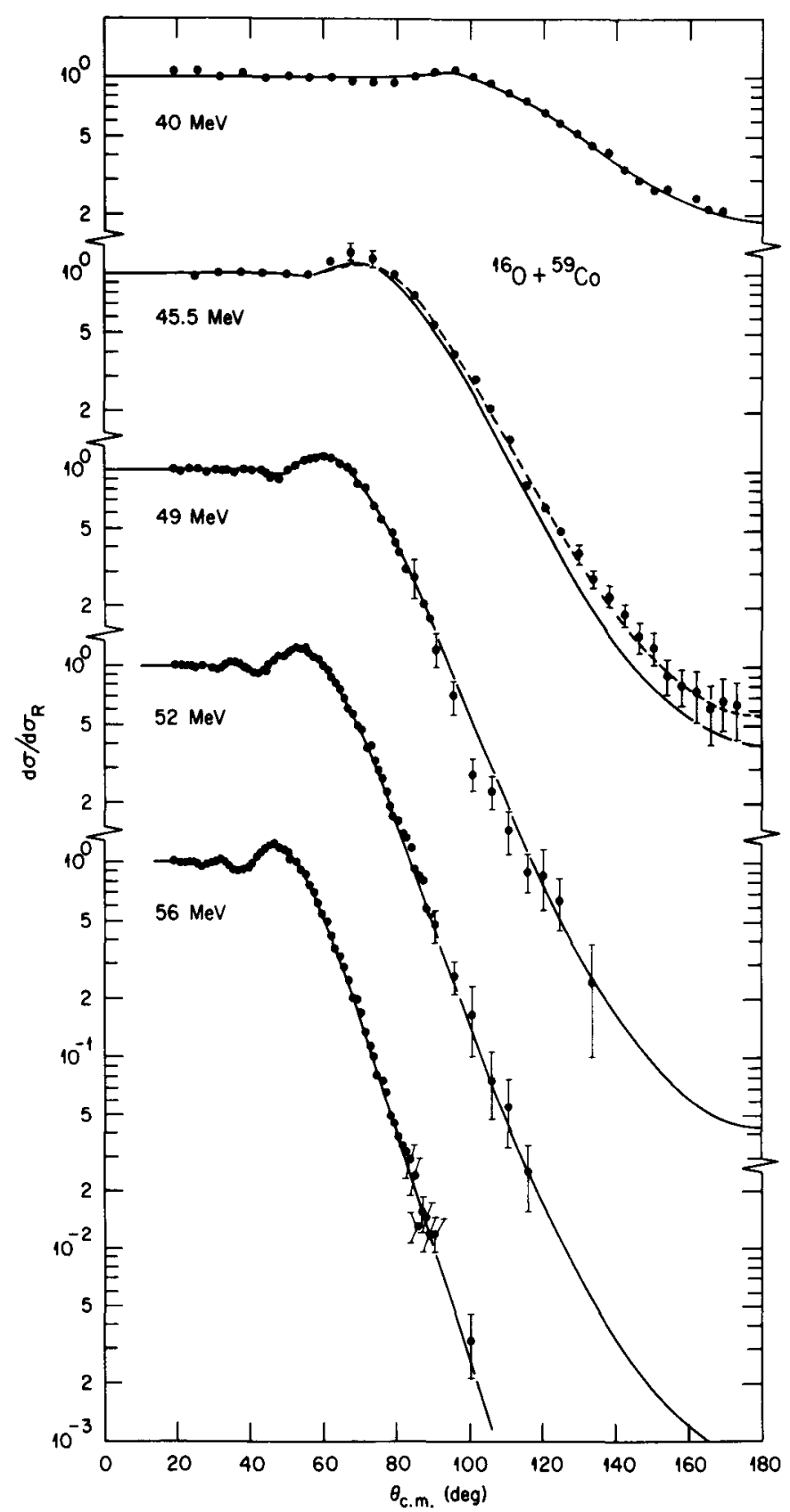

Fig. 12. Comparison with data for ${ }^{16} \mathrm{O}+{ }^{59} \mathrm{Co}$ at various energies. The solid curves are for $N=1.0, W=12 \mathrm{MeV}, r_{\mathrm{W}}=1.3 \mathrm{fm}$, $a_{\mathrm{W}}=0.50 \mathrm{fm}$, while the dashed curve is for the potential listed in table 3 for $45.5 \mathrm{MeV}$.

potential parameters (table 3) were obtained by fitting the scattering at $143.6 \mathrm{MeV}$. The data at the other energies are not complete enough to determine potential parameters in a meaningful way, but they are in reasonable agreement with the predicted cross sections. The $N$ value of 1.32 deduced is rather large, and might be taken as indicating that Negele's density for ${ }^{40} \mathrm{Ca}$ is somewhat too small. For example, his calculations result in a neutron distribution with a slightly smaller radius than the proton one (table 1). If we take the neutrons to have the same distribution that he 


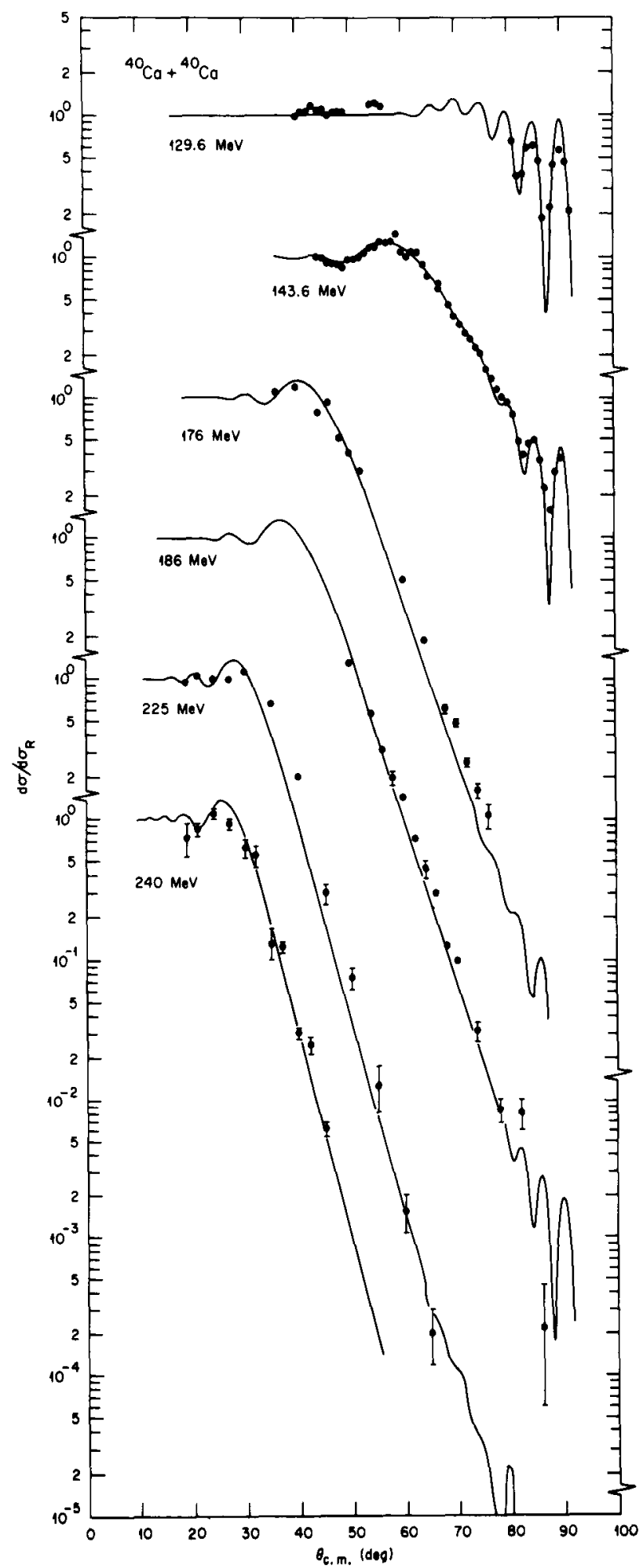

Fig. 13. The scattering of ${ }^{40} \mathrm{Ca}+{ }^{40} \mathrm{Ca}$. The curves are predictions using the potential (table 3) fitted to the data at $143.6 \mathrm{MeV}$. 
finds for the protons, the folded potential in this case is increased by $10 \%$ at the strong absorption radius, which would reduce $N$ to about 1.20 . (The changes for other systems involving only one ${ }^{40} \mathrm{Ca}$ nucleus would be about half this.)

It is also interesting to note that the density-dependent DDD interaction gives almost the same folded potential for ${ }^{40} \mathrm{Ca}+{ }^{40} \mathrm{Ca}$ as the M3Y interaction; at the strong absorption radius the DDD one is $6 \%$ stronger.

Results for some other systems of comparable mass with ${ }^{32} \mathrm{~S}$ as projectile are illustrated in fig. 14. We were unable to fit accurately both the oscillation about the Rutherford cross section and the fall-off in the shadow region for ${ }^{32} \mathrm{~S}+{ }^{40} \mathrm{Ca}$; the minimum $\chi^{2}$ fit shown is a compromise in this respect. We note that here $N$ for ${ }^{48} \mathrm{Ca}$ (table 3 ) is $13 \%$ smaller than for ${ }^{40} \mathrm{Ca}$, just as it was for ${ }^{16} \mathrm{O}$ as projectile. The fit to ${ }^{32} \mathrm{~S}+{ }^{32} \mathrm{~S}$ is satisfactory but is not very sensitive to the value of $N$; further, there seems to be some uncertainty $(\lesssim 10 \%)$ in the absolute normalization of the data. The parameters given in table 3 are representative.

All of the systems discussed here seem to require an absorptive potential with a sharper edge (smaller $a_{\mathrm{w}}$ value) than is usually necessary for lighter projectiles.

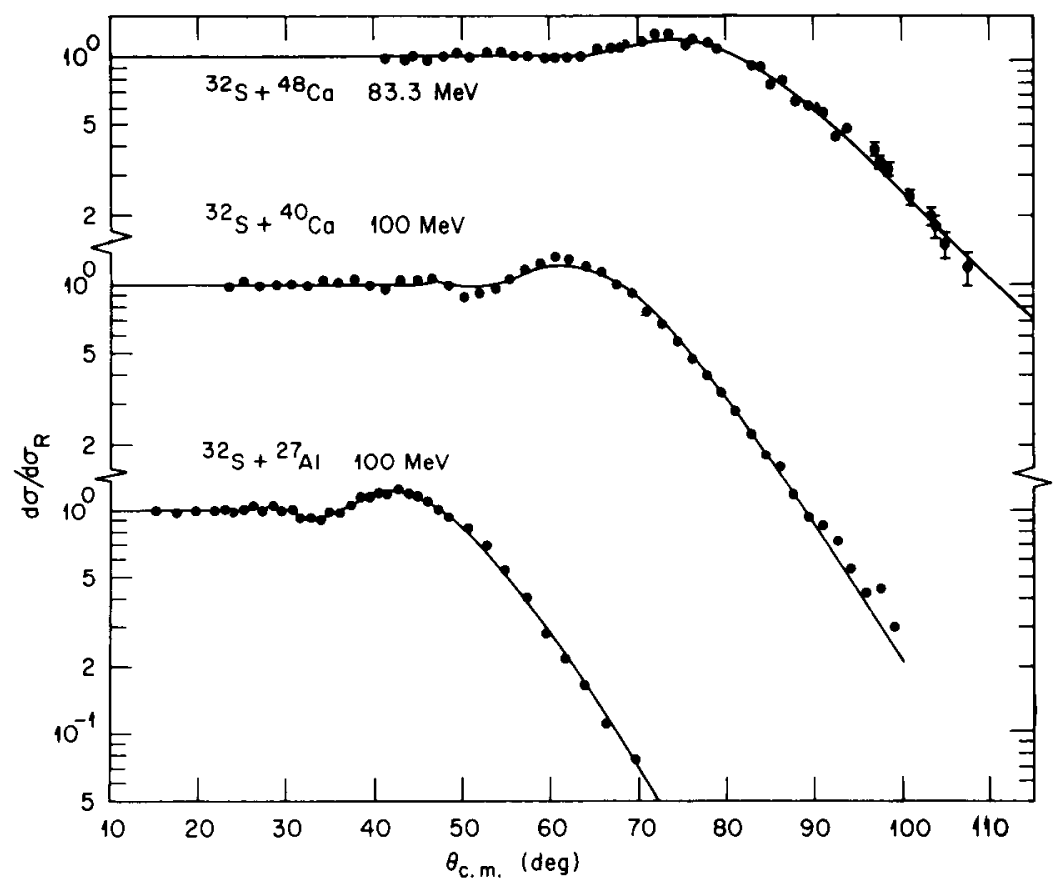

Fig. 14. The scattering of ${ }^{32} \mathrm{~S}$ from several targets. The curves were obtained by fitting the data (table 3 ).

\subsubsection{The target ${ }^{208} \mathrm{~Pb}$}

There are many measurements of ${ }^{16} \mathrm{O}+{ }^{208} \mathrm{~Pb}$ from the Coulomb barrier $(\sim 80 \mathrm{MeV})$ up to $313 \mathrm{MeV}$ (or $E / A$ of 5 to $20 \mathrm{MeV}$ ) so that this would seem to be an ideal system for testing the folded potential. Unfortunately, there seem to be many inconsistencies between these sets of data, resulting in fluctuations in the potential parameters needed to fit them (see $[47,102,103]$ for example). Consequently, it seems impossible to learn anything unambiguously such as, for example, whether there is evidence for an energy dependence. Fits to the data for 129.5 and $192 \mathrm{MeV}$ [47] 


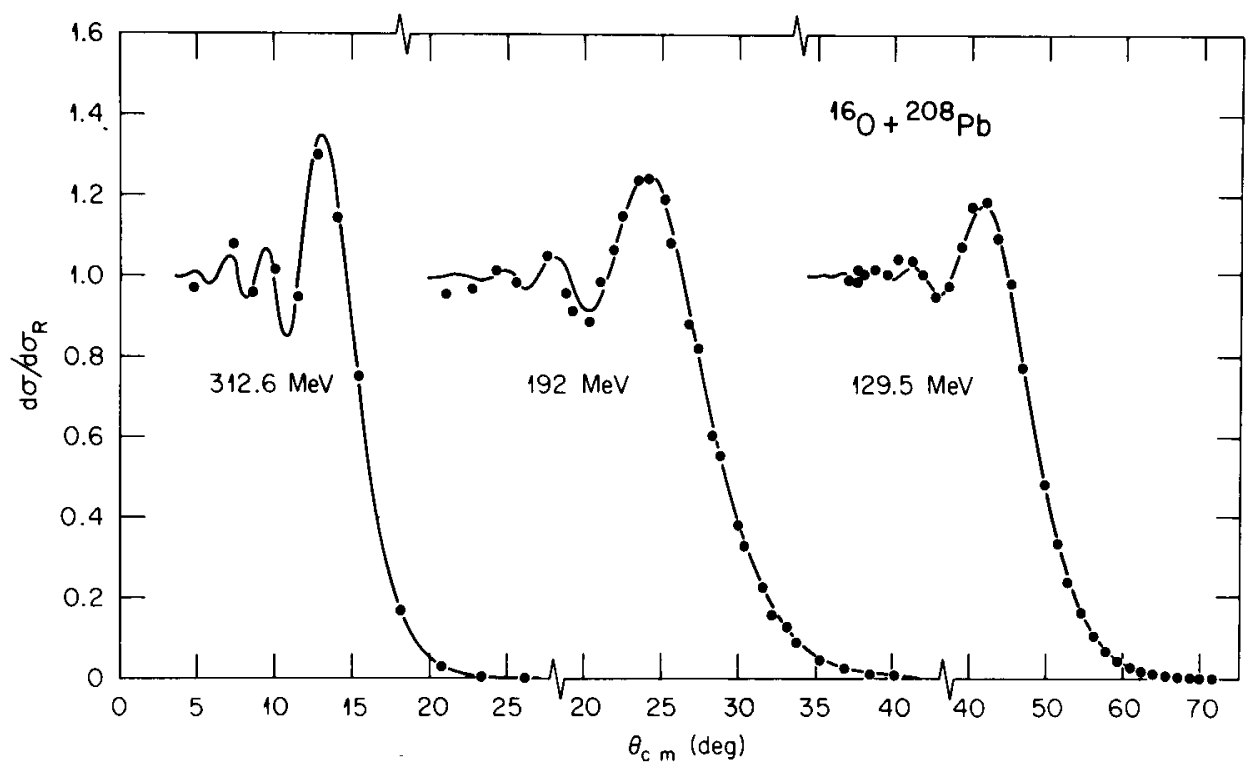

Fig. 15. The scattering of ${ }^{16} \mathrm{O}+{ }^{208} \mathrm{~Pb}$ at three energies. The curves are fits obtained with the parameters of table 3 .

and for $312.6 \mathrm{MeV}$ [103] are shown in fig. 15, with the parameters given in table 3 . The optimum $N$ values are close to unity, $N=1.05 \pm 0.09$. The $129.5-\mathrm{MeV}$ data seem to demand a real potential about $20 \%$ stronger than needed at $192 \mathrm{MeV}$, whatever form of potential is used [47]. We believe this is an idiosyncracy of the data (perhaps due to small errors in absolute normalization), not a true energy dependence; there is no evidence for such a strong energy dependence from analysis of the data at other energies. Table 3 also seems to indicate a strong energy dependence for $W$, but again the data at other energies do not support this.

Figure 16 shows fits for ${ }^{12} \mathrm{C}+{ }^{208} \mathrm{~Pb}$ at 96 and $116.4 \mathrm{MeV}$; the $96-\mathrm{MeV}$ potential (table 3) also gives an acceptable fit to other data at $78 \mathrm{MeV}$. The $N$ value of 1.25 is a little high; similar values are obtained (table 3) for ${ }^{11} \mathrm{~B}+{ }^{208} \mathrm{~Pb}$ and ${ }^{13} \mathrm{C}+{ }^{207} \mathrm{~Pb}$, and we have already remarked earlier that ${ }^{11} \mathrm{~B}+{ }^{209} \mathrm{Bi}$ requires $N=1.45$. It remains to be seen whether these values represent inadequacies in the density distributions used or whether perhaps they are a consequence of the collectivity of ${ }^{12} \mathrm{C}$-like nuclei. However, it is interesting to note that these particular discrepancies are largely removed if we use the alternate shell-model density distribution for ${ }^{208} \mathrm{~Pb}$ that was described in section 3.4.4. For this, the neutron distribution was generated using a potential well with the same Batty-Greenlees dimensions as was used for the protons and resulted in a difference of $0.29 \mathrm{fm}$ between the neutron and proton RMS radii. This new density increases the folded potentials near the strong absorption radii by 20 to $25 \%$ and results in optimum $N$ values of 1.17 , 1.01 and 1.05 for ${ }^{11} \mathrm{~B},{ }^{12} \mathrm{C}$ and ${ }^{13} \mathrm{C}$, respectively. However, the optimum $N$ values for ${ }^{16} \mathrm{O}$ are then reduced to $0.94,0.78$ and 0.87 at $129.5,192$ and $312.6 \mathrm{MeV}$, respectively, so that the discrepancies between ${ }^{16} \mathrm{O}$ and the ${ }^{12} \mathrm{C}$-like projectiles remain at approximately $20 \%$.

\subsubsection{Some other systems}

The scattering of ${ }^{12} \mathrm{C}$ from ${ }^{40} \mathrm{Ca}$ and ${ }^{90} \mathrm{Zr}$ is reasonably well reproduced (fig. 17). In both cases some diffraction structure is seen at larger angles although only the ${ }^{40} \mathrm{Ca}$ data show evidence for this. 


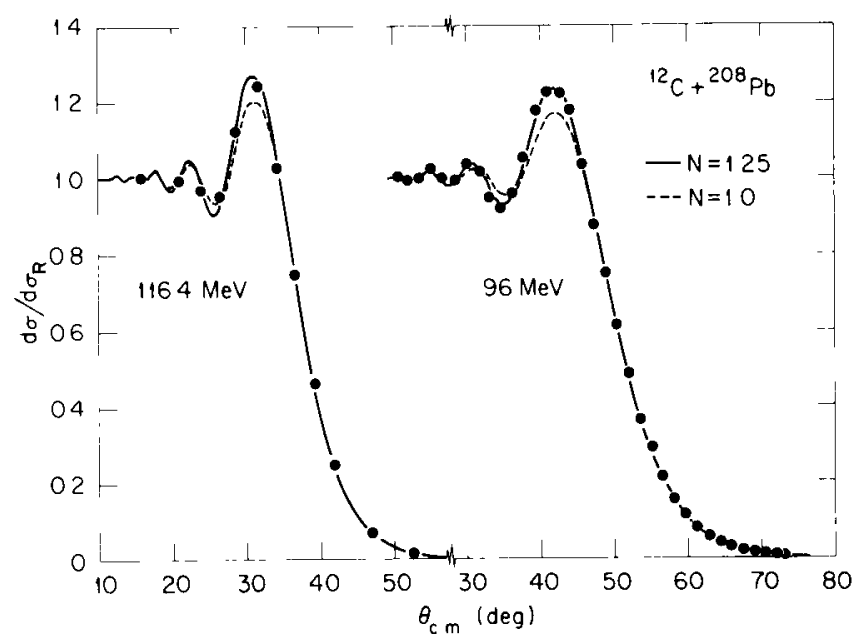

Fig. 16. The scattering of ${ }^{12} \mathrm{C}+{ }^{208} \mathrm{~Pb}$ at two energies. The solid curves are both obtained with the optimum potential for the $96-\mathrm{MeV}$ data (table 3 ), while the dashed curves are for fixed $N=1.0$ but with the imaginary potential strength and diffuseness parameters optimized $\left(W=12 \mathrm{MeV}, a_{\mathrm{W}}=0.635 \mathrm{fm}\right)$.

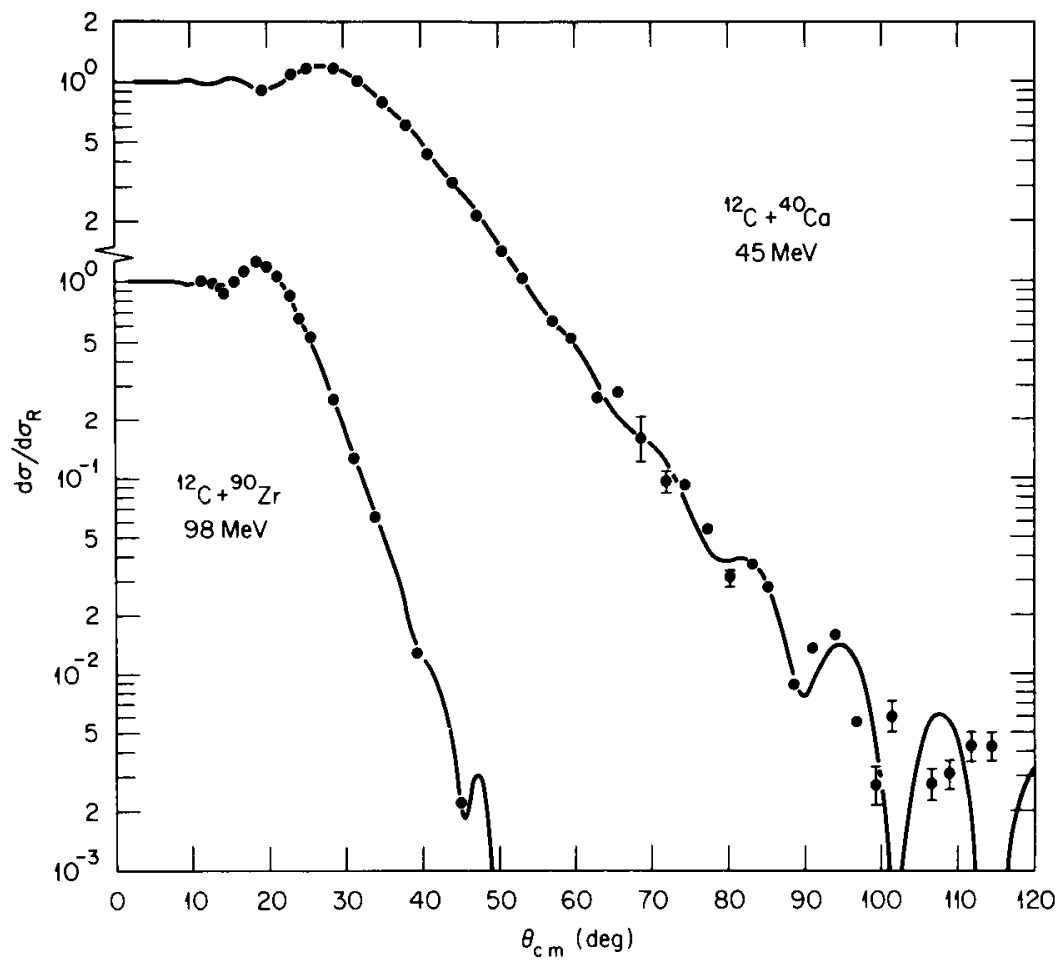

Fig. 17. The scattering of ${ }^{12} \mathrm{C}+{ }^{40} \mathrm{Ca},{ }^{90} \mathrm{Zr}$. The curves are fits to the data (table 3 ).

Figure 18 shows results for ${ }^{16} \mathrm{O}+{ }^{88} \mathrm{Sr}$ and ${ }^{15} \mathrm{~N}+{ }^{89} \mathrm{Y}$. These data require $N \approx 1.2$ (the $\chi^{2}$ minimum gives $N=1.36$ for ${ }^{16} \mathrm{O}+{ }^{88} \mathrm{Sr}$ at $48 \mathrm{MeV}$, but $N \approx 1.2$ gives an equally acceptable fit). The other noteworthy feature is the need for a small diffuseness $\left(a_{\mathrm{w}} \lesssim 0.4 \mathrm{fm}\right)$ for the imaginary potential in these cases. 


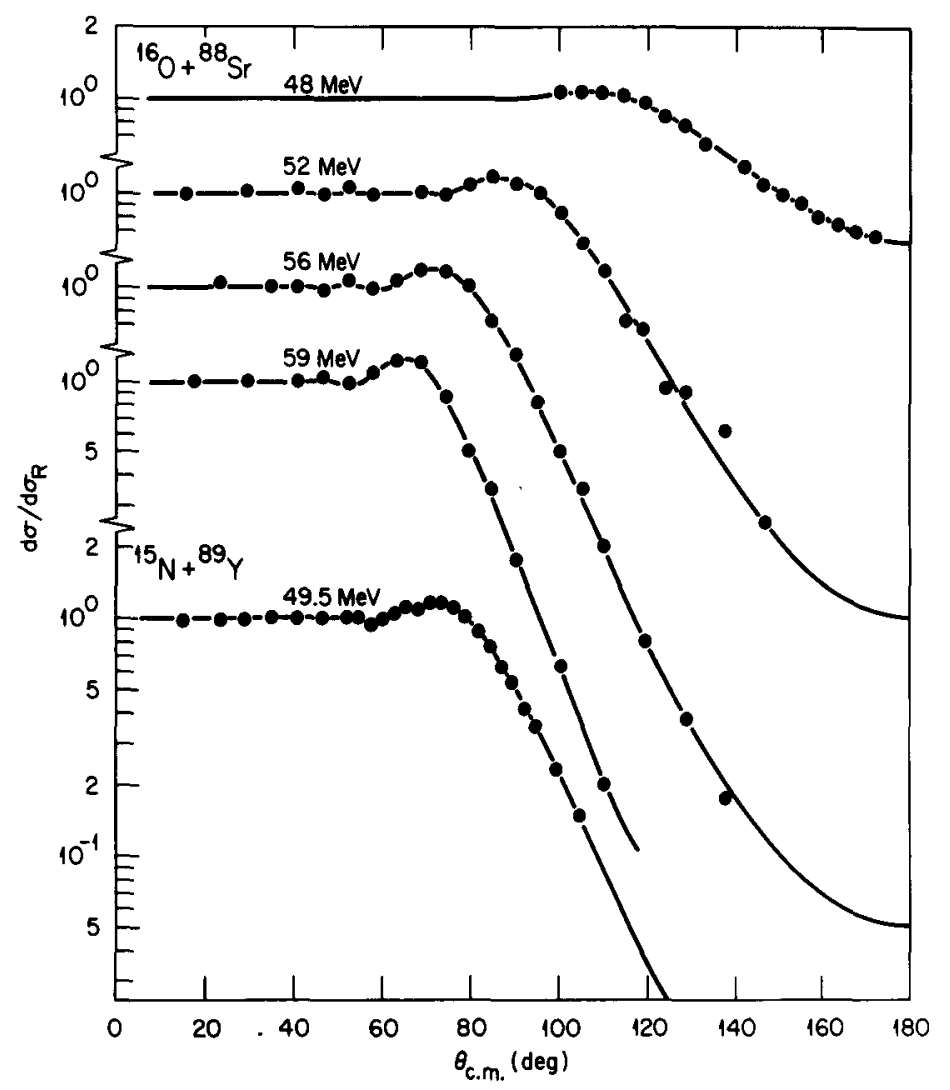

Fig. 18. The scattering of ${ }^{16} \mathrm{O}+{ }^{88} \mathrm{Sr}$ at several energies and of ${ }^{15} \mathrm{~N}+{ }^{89} \mathrm{Y}$. The curves are fits to the data (table 3).

\subsubsection{The target ${ }^{28} \mathrm{Si}$}

This target has received special attention recently because of the discovery of the "anomalous large-angle scattering" (see $[15,100]$ for example) of ${ }^{12} \mathrm{C}$ and ${ }^{16} \mathrm{O}$ from this nucleus. Earlier analyses with a folded potential [94] of extensive data for ${ }^{16} \mathrm{O}+{ }^{28} \mathrm{Si}$ at energies from 33 to $215 \mathrm{MeV}$ showed that the overall features of the forward scattering data could be fitted; a reanalysis of the 141.5- and 215.2-MeV data with the M3Y interaction (10) used here gives similar results (table 3). In both cases we find $N<1.0$ and an imaginary potential with small $r_{\mathrm{W}} \approx 0.9 \mathrm{fm}$ and large $a_{\mathrm{W}} \approx 1.0 \mathrm{fm}$ is required. A similar potential (table 3) fits the $131.5-\mathrm{MeV}$ data for ${ }^{12} \mathrm{C}+{ }^{28} \mathrm{Si}$. (None of these high-energy data extend into the small angles where the cross section oscillates about the Rutherford value; consequently, this important constraint on the potential is missing.)

However, none of these earlier fits reproduced the oscillatory structure seen at the larger angles and for energies from 50 to $70 \mathrm{MeV}$; this structure was confirmed beautifully by later measurements [104]. Then data became available [105] for angles out to nearly $180^{\circ}$, revealing the "anomalous large-angle scattering".

We have not yet succeeded in obtaining a good fit to these new data. However, earlier we examined some of the data for energies from 53 to $81 \mathrm{MeV}$, restricting attention to angles less than $50^{\circ}$, as well as the whole angular range at $37.7 \mathrm{MeV}$. The intent was to see whether our "standard" imaginary potential with $r_{\mathrm{W}}=1.3 \mathrm{fm}$ and $a_{\mathrm{W}}=0.6 \mathrm{fm}$ could be used, instead of the somewhat unusual parameters required for the high-energy fits, and what the resulting $N$ values would be. 
Indeed good fits were obtained with optimum $N \approx 1.0$ and with imaginary strengths $W$ of about $8 \mathrm{MeV}$; the parameters are included in table 3 . The rather small value of $W$ suggests the possibility of large-angle effects from the internally reflected waves $[26,71]$ and indeed examination shows that these potentials do predict scattering analogous to that seen experimentally [105]. The cross sections at $180^{\circ}$ are of order $10^{-2}$ of the Rutherford value, although the oscillations at large angles do not completely match the ones observed; it will be important for our understanding of the potential to see what further modification is required to fit the data.

A recent analysis [106] of the 50 - and $55-\mathrm{MeV}$ data at forward angles using a Woods-Saxon potential form concluded that the real potential was $-0.75 \mathrm{MeV}$ at $R=9.02 \mathrm{fm}$ (50-MeV data) and $-0.8 \mathrm{MeV}$ at $R=8.92 \mathrm{fm}(55-\mathrm{MeV}$ data). These values may be compared to our folded potential values (with $N=1.0$ ) of -0.70 and $-0.80 \mathrm{MeV}$, respectively. The authors then showed that a reasonable fit to the large-angle data could be obtained with potentials constrained to be equal to these values. In other words, our folded potential is correct at large separations $R \gtrsim 8 \mathrm{fm}$; it remains to be seen whether the large-angle data can be explained by adjustment only of the imaginary potential (e.g. by making it $L$-dependent) without modifying the real folded potential at smaller $R$.

\section{Scattering of ${ }^{6,7} \mathrm{Li}$ and ${ }^{9} \mathrm{Be}$}

The scattering of $\mathrm{Li}$ ions is of particular interest because ${ }^{6} \mathrm{Li}$ appears to be "anomalous" in the sense that folded potentials for this projectile, using the same interaction which we have just seen to be successful for many other ions, need to be renormalized by $N \approx 0.6$ [73]. This is a substantial deviation from unity and is necessary for essentially all the targets studied thus far.

We have made only a few studies of ${ }^{7} \mathrm{Li}$ scattering, from ${ }^{40,48} \mathrm{Ca}$ at 28 and $34 \mathrm{MeV}$, and from ${ }^{90} \mathrm{Zr}$ at $34 \mathrm{MeV}$, but we see no evidence that this isotope of $\mathrm{Li}$ is "anomalous" in the same way. The results are included in table 4; in each case the value of $N$ is close to unity. We note also that in each case an appreciable improvement in fit is obtained by allowing the value of $a_{\mathrm{w}}$ to be larger than $0.6 \mathrm{fm}$.

\subsection{Density distributions for Li nuclei}

\subsubsection{The ${ }^{6} \mathrm{Li}$ nucleus}

Because of the suggestion [73] that ${ }^{6} \mathrm{Li}$ scattering is anomalous, particular care was taken to explore several ways of constructing density distributions for this nucleus. The first was to take the proton distribution from the charge distribution determined by electron scattering [124] and then assume that the neutron distribution was the same. The charge distribution used [124] was

$$
\rho_{\mathrm{ch}}(r)=\frac{3}{8 \pi^{3 / 2}}\left[\frac{1}{a^{3}} \exp \left(-\frac{r^{2}}{4 a^{2}}\right)-\frac{c^{2}\left(6 b^{2}-r^{2}\right)}{4 b^{7}} \exp \left(-\frac{r^{2}}{4 b^{2}}\right)\right]
$$

with $a=0.928 \mathrm{fm}, b=1.26 \mathrm{fm}$ and $c=0.48 \mathrm{fm}$. This corresponds to an RMS charge radius of $2.56 \mathrm{fm}$. The finite size of the neutron and proton charge distributions was unfolded as discussed in section 3.4.1 so as to give the distribution of the centers of mass of the protons. The latter then has an RMS radius of $2.43 \mathrm{fm}$. 


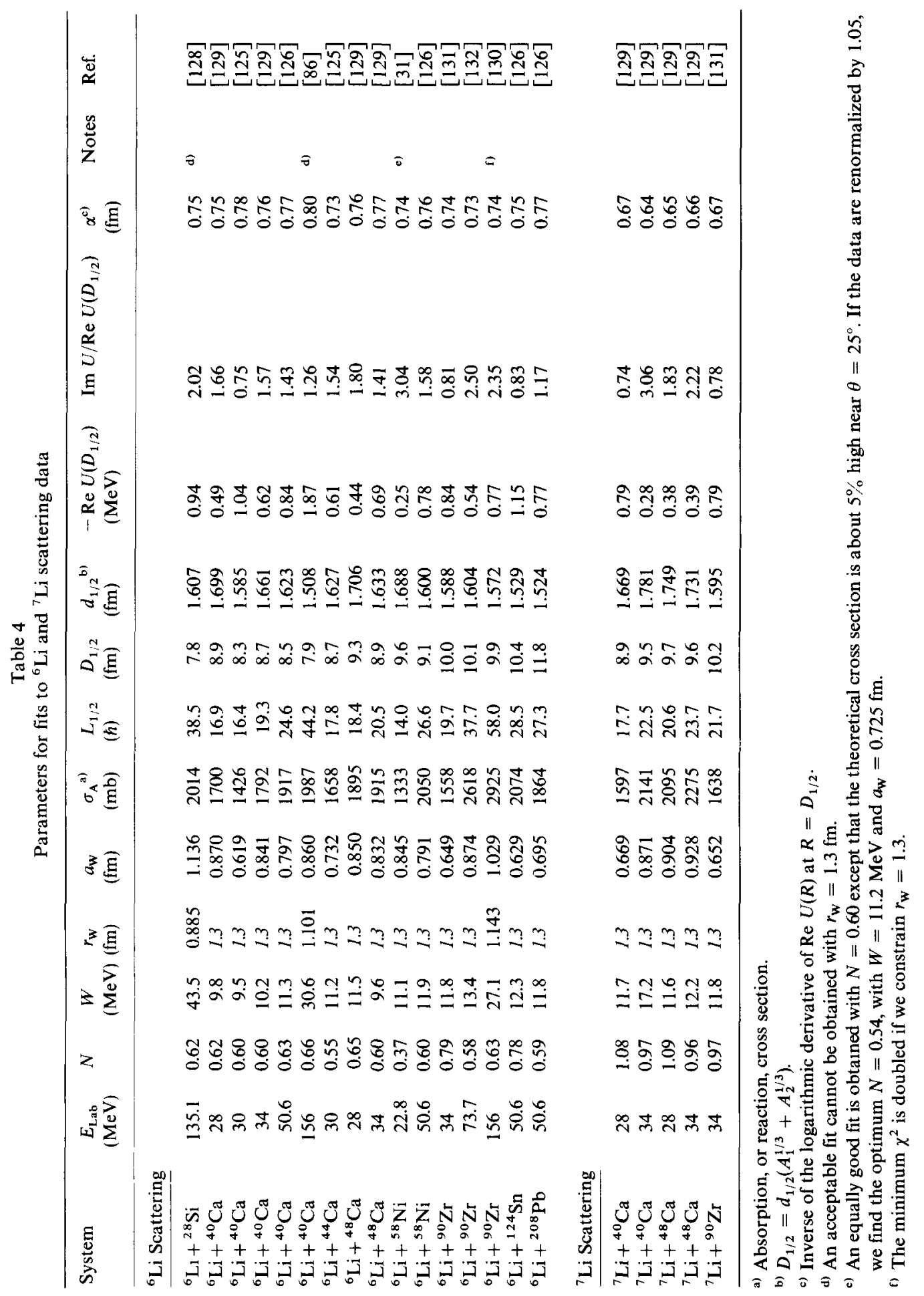


In addition, proton distributions were constructed from shell model wave functions in three different ways. In each case a configuration $\left(1 \mathrm{~s}_{1 / 2}^{2} 1 \mathrm{p}_{3 / 2}\right)$ was assumed and the shell model WoodsSaxon potential well was taken to have a surface diffuseness $a=0.65 \mathrm{fm}$ with a spin-orbit coupling of 17 times the Thomas term. Then the radius of the well was adjusted so that the resulting proton distribution had an RMS radius of $2.43 \mathrm{fm}$. We assumed that the neutron distribution was the same as for the protons.

First, the protons were assumed bound by their observed separation energies $S\left(\mathrm{~s}_{1 / 2}\right)=22.7 \mathrm{MeV}$, $S\left(\mathrm{p}_{3 / 2}\right)=4.6 \mathrm{MeV}$, and no account was taken of the center of mass recoil; this required $r_{0}=1.40 \mathrm{fm}$. This prescription gives an incorrect tail to the distribution [62], so in the next attempt the center of mass recoil was corrected for by unfolding recoil motion with a mean square radius of $0.6 \mathrm{fm}^{2}$. We then needed a well with $r_{0}=1.59 \mathrm{fm}$ in order to get the required RMS radius. Finally, a third prescription used was to include the recoil effects by taking the binding energies to be $(6 / 5)^{2}$ times the separation energies; this gives the correct form for the tail of the distribution [62], although it is not quite "correct" in the nuclear interior. This prescription needed a well with $r_{0}=1.65 \mathrm{fm}$.

It turns out that these various approaches give very similar density distributions. The resulting folded potentials are even more similar, providing further evidence that the mean square radius of the density is the critical quantity. Near the strong absorption radii, the various prescriptions give potentials which differ by $3 \%$ or less.

One uncertainty is whether the neutron distribution differs from the proton one. The importance of this was tested in one way, by assuming that the neutron shell model potential had the same shape as the proton one but with its depth adjusted to give neutron binding energies about $1 \mathrm{MeV}$ larger than those for the protons. This gives a neutron RMS radius about $0.04 \mathrm{fm}$ smaller than the proton one and a folded potential which is a few per cent weaker at the strong absorption radius.

One of the density distributions just described was based upon the charge distribution obtained from electron scattering. Further, the folded potentials obtained are determined primarily by the mean square radius of the ${ }^{6} \mathrm{Li}$ distribution rather than its detailed shape, provided the latter is "reasonable". Hence other structure models for ${ }^{6} \mathrm{Li}$, such as an $\alpha+\mathrm{d}$ cluster, can be expected to give similar results. Consequently, it does not seem likely that such a large "anomaly" in the folded potentials for ${ }^{6} \mathrm{Li}$ could be due to an unwise choice of density distribution for that nucleus.

\subsubsection{The ${ }^{7}$ Li nucleus}

The shell model was used for both neutrons and protons since there was no reason to assume here that $\rho_{\mathrm{n}}=(N / Z) \rho_{\mathrm{p}}$. Configurations of $\left(1 \mathrm{~s}_{1 / 2}^{2} 1 \mathrm{p}_{3 / 2}\right)$ (protons) and $\left(1 \mathrm{~s}_{1 / 2}^{2} 1 \mathrm{p}_{3 / 2}^{2}\right)$ (neutrons) were assumed. The $1 \mathrm{p}_{3 / 2}$ binding energies were taken as $(7 / 6)^{2}$ times the averages of the separation energies which leave ${ }^{6} \mathrm{Li}$ or ${ }^{6} \mathrm{He}$, respectively, in their ground and first excited states [62]: $\bar{S}\left(1 \mathrm{p}_{3 / 2}\right)=8.35 \mathrm{MeV}$ (neutron), $10.9 \mathrm{MeV}$ (proton). The $1 \mathrm{~s}_{1 / 2}$ nucleons were bound by $25 \mathrm{MeV}$ (neutron) or $23 \mathrm{MeV}$ (proton). The potential well radius was then adjusted to give an RMS radius of $2.28 \mathrm{fm}$ for the protons, corresponding to a charge radius of $2.41 \mathrm{fm}$ [59]. This required $r_{0}=1.475$ fm and the same value was used for the neutrons. Then the RMS radius for the neutrons was $2.44 \mathrm{fm}$, or $0.16 \mathrm{fm}$ larger than for the protons.

Again, very similar results were obtained by binding the nucleons by their separation energies and unfolding the center of mass recoil explicitly. For example, this prescription gives a folded potential for ${ }^{7} \mathrm{Li}+{ }^{40} \mathrm{Ca}$ which is about $10 \%$ deeper at the strong absorption radius.

It is possible that our approach has overestimated the difference in the neutron and proton RMS radii and that the neutron-proton correlations actually reduce this difference. If that is the 
case, we will also have overestimated somewhat the folded potentials near the strong absorption radii for ${ }^{7} \mathrm{Li}$ scattering so that the correct $N$ values for ${ }^{7} \mathrm{Li}$ should be larger than those shown in table 4.

\subsection{Results for ${ }^{6} \mathrm{Li}$ scattering}

Table 4 gives the optimum parameters for fits to ${ }^{6} \mathrm{Li}$ scattering data. (The results reported there were obtained using the first of the shell model prescriptions for ${ }^{6} \mathrm{Li}$ described in section 7.2.1.) We kept the imaginary radius parameter $r_{\mathrm{w}}=1.3 \mathrm{fm}$ except for those few cases, all for high energy, where the data demanded a smaller value. In every case a significant improvement was obtained by allowing the value of $a_{\mathrm{w}}$ to be greater than $0.6 \mathrm{fm}$.

The values of $N$ are appreciably less than unity. The optimum value of $N$ for ${ }^{58} \mathrm{Ni}$ at $22.8 \mathrm{MeV}$ is low, but a $5 \%$ increase in absolute normalization of these data raises it to $N=0.54$. The values for ${ }^{124} \mathrm{Sn}$ and for ${ }^{90} \mathrm{Zr}$ at $34 \mathrm{MeV}$ are somewhat high (the latter case being somewhat out of line compared with the results for ${ }^{90} \mathrm{Zr}$ at the higher energies), but the remaining values cluster around $\bar{N} \approx 0.62$. We can conclude that the double-folding model, with the M3Y interaction which is found to be satisfactory for many other heavy ion systems (table 3), overestimates the potential for ${ }^{6} \mathrm{Li}$ scattering by about $60 \%$.

We have already remarked that values of $a_{\mathrm{w}}$ greater than $0.6 \mathrm{fm}$ are needed for ${ }^{6} \mathrm{Li}$; this is to be contrasted with the imaginary potentials for heavier ions (table 3) where either $a_{\mathrm{w}}=0.6 \mathrm{fm}$ is adequate or else smaller values are required. The behaviour of the ${ }^{6} \mathrm{Li}$ potentials is reminiscent of those for deuteron scattering and we may surmise that it is for the same reasons, weak binding and ease of break-up. We may also note from table 4 that the slopes of the real folded potentials (represented by $\alpha$ ) at the strong absorption radii are less for ${ }^{6} \mathrm{Li}(\alpha$ between 0.7 and $0.8 \mathrm{fm}$ ) than for the heavier ions ( $\alpha$ between 0.6 and $0.7 \mathrm{fm}$ ). This is also associated with the weaker binding of ${ }^{6} \mathrm{Li}$.

\subsection{The case of ${ }^{6} \mathrm{Li}+{ }^{40} \mathrm{Ca}$}

Most of the ${ }^{6} \mathrm{Li}$ scattering considered here is sensitive only to the potential in the vicinity of the strong absorption radius. An important exception is for the target ${ }^{40} \mathrm{Ca}$ at $30 \mathrm{MeV}$. These data [125] extend back to about $175^{\circ}$ (see fig. 19) and show "anomalous large-angle scattering" which is analogous to, although not as marked as, that discussed earlier for $\alpha+{ }^{40} \mathrm{Ca}$ and ${ }^{16} \mathrm{O}+{ }^{28} \mathrm{Si}$. In particular, the cross sections at large angles are more than an order of magnitude larger than those for ${ }^{6} \mathrm{Li}+{ }^{44} \mathrm{Ca}$ at the same energy. As fig. 19 shows, the folded potential is able to give a good fit to these data. It also fits those for ${ }^{44} \mathrm{Ca}$. The main difference is that the absorptive potential is weaker for ${ }^{40} \mathrm{Ca}$ (both $W$ and $a_{\mathrm{w}}$ are smaller than for ${ }^{44} \mathrm{Ca}$ ).

The same characteristic was observed for the other "anomalous large-angle scattering" cases and implies [26,71] some degree of penetration into the interior, $R \ll D_{1 / 2}$. It is this aspect which is of especial interest in the present context. The scattering of ${ }^{6} \mathrm{Li}+{ }^{40} \mathrm{Ca}$ at $30 \mathrm{MeV}$ is found to be sensitive to the real potential into $R \approx 3 \mathrm{fm}$, corresponding to considerable overlap of the densities of the two ions. Yet the data can be fitted by a simple renormalization of this potential by a radially-independent factor $N \approx 0.6$.

The scattering of ${ }^{6} \mathrm{Li}+{ }^{40} \mathrm{Ca}$ at $156 \mathrm{MeV}$, also shown in fig. 19 , still requires $N \approx 0.6$ but is less sensitive to the interior of the potential. (Figure 19 also shows that in this case it is not satisfactory to use an imaginary potential with the same shape as the real, folded, one; i.e. it is not 
sufficient to use a complex $N$.) The data for the energies close to $30 \mathrm{MeV}$, namely 28 and $34 \mathrm{MeV}$, are a little puzzling. Both indicate $N \approx 0.6$ for the real potential but seem to demand a larger imaginary diffuseness parameter $a_{\mathrm{w}}$ than at $30 \mathrm{MeV}$.

\subsection{Discussion}

We have presented evidence that ${ }^{6} \mathrm{Li}$ behaves differently from the other ions we have considered, even from ${ }^{7} \mathrm{Li}$, in that the double-folding model with the M3Y interaction gives potentials which are almost a factor of two too strong. The nucleus ${ }^{6} \mathrm{Li}$ certainly differs from the other projectiles in two conspicuous respects: (i) it has a spin of 1 , and (ii) it is more weakly bound. Other calculations $[31,93,126]$ have indicated that a reasonable strength of spin-orbit coupling has rather small effects on the differential cross sections. We verified that this was so even for the backward angles for ${ }^{40} \mathrm{Ca}$ at $30 \mathrm{MeV}$. Hence we believe that neglecting the spin-orbit potential is not responsible for the discrepancy. Further, the spin-spin part $v_{10}$ of the central interaction (6) cannot contribute to the folded potential for a spin-zero target (appendix A).

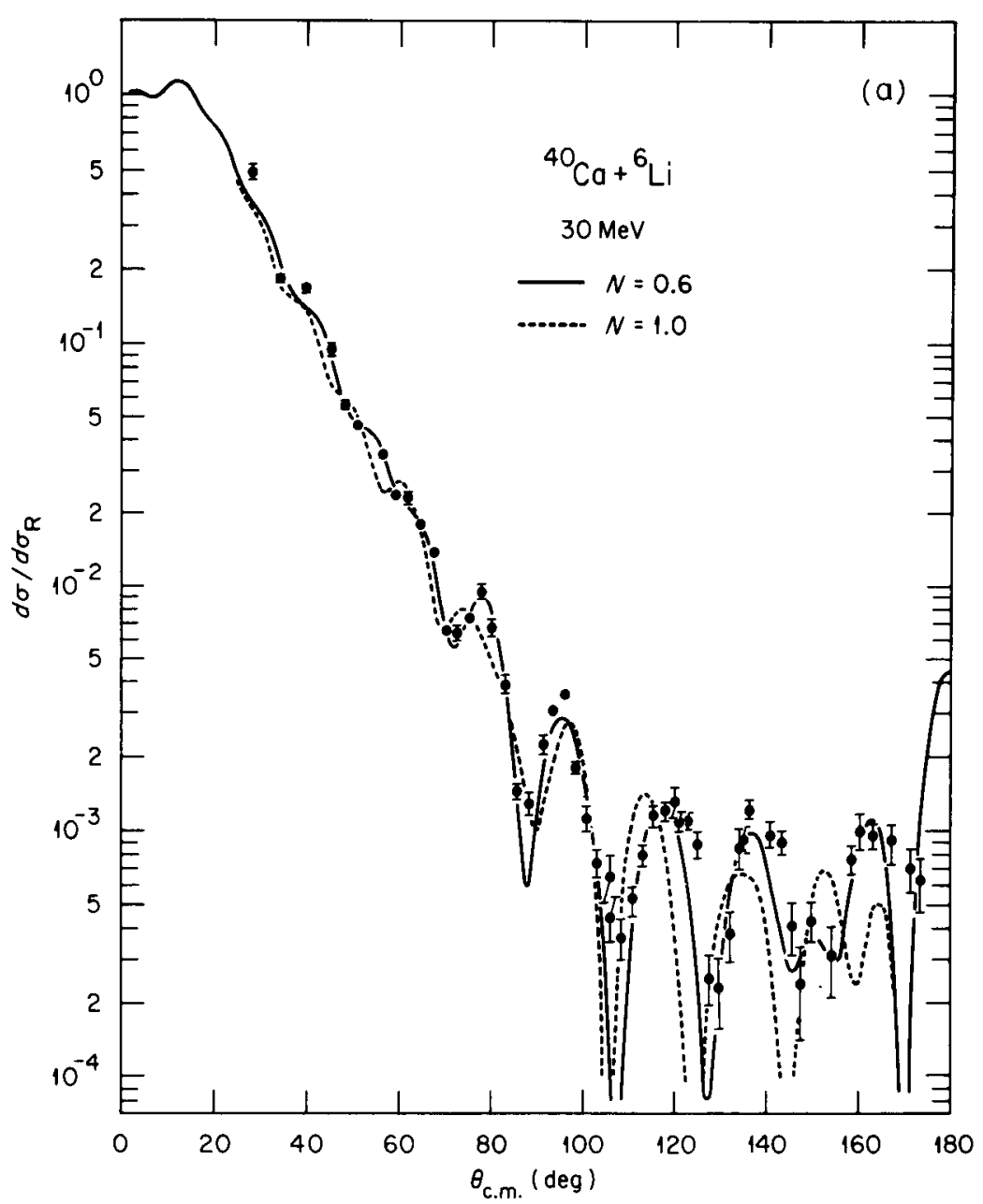


A more likely explanation would seem to be the polarization and break-up of the ${ }^{6} \mathrm{Li}$ in the field of the target nucleus [127] which could make important contributions to the $\Delta U$ term of eq. (3). However, there is no clear evidence from table 4 that the deviation of $N$ from unity increases as the target mass increases, which suggests at least that it is not the Coulomb interaction which is responsible. Further, there is no indication that the renormalization needed varies with the bombarding energy. At first sight, the sign of the effect is a little puzzling. A perturbative treatment results in the real part of the polarization potential being attractive in both the adiabatic and the sudden limits [24] whereas the observed effect is repulsive. The ${ }^{6} \mathrm{Li}$ behaves as though it were smaller than is indicated by electron scattering measurements [124].

\subsection{The ${ }^{9} \mathrm{Be}$ nucleus}

Since this review was originally prepared, the scattering of ${ }^{9} \mathrm{Be}$ from a number of targets has been studied [133]. Here we report briefly on the results since ${ }^{9} \mathrm{Be}$ appears to offer an "anomaly" similar to that found for ${ }^{6} \mathrm{Li}$.

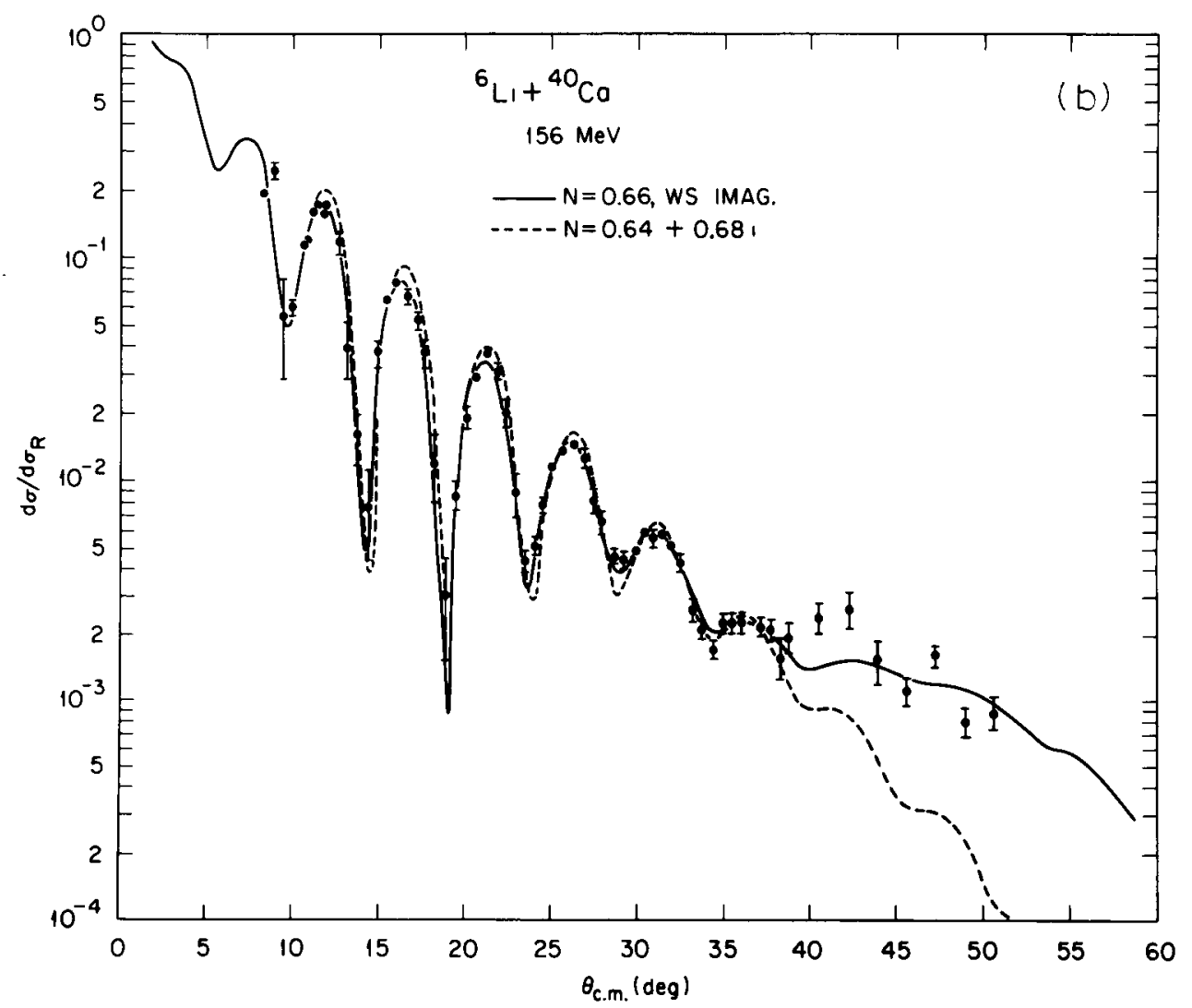

Fig. 19. The scattering of ${ }^{6} \mathrm{Li}+{ }^{40} \mathrm{Ca}$ at two energies. The solid curves are fits to the data (table 4). The dashed curve at $30 \mathrm{MeV}$ is the result of constraining $N=1.0$ while optimizing the imaginary potential parameters. The dashed curve at $156 \mathrm{MeV}$ is a two-parameter fit in which the imaginary potential was taken to have the same shape as the folded real potential. 


\subsubsection{Density distribution for ${ }^{9} \mathrm{Be}$}

A crucial ingredient in the calculation of the folded potential is the one-body density distribution for ${ }^{9} \mathrm{Be}$. Electron scattering [134] gives a precise value for the charge rms radius $(2.519 \pm 0.012 \mathrm{fm})$ which places a very important constraint on the proton distribution. However, there is no independent information on the neutron distribution and this is important because of the very weak binding $(1.67 \mathrm{MeV})$ of the odd neutron. We approached this problem in several ways, each being a variation of the independent-particle model and an extension of what was done for ${ }^{6} \mathrm{Li}$.

A density distribution can be constructed in principle from the same radial overlap factors that appear in treatments of nucleon pick-up reactions [62]. However, the construction of these overlap factors in general is not simple [135]. We adopted the widely used prescription that their radial shapes could be represented by the wave function of a nucleon in a Woods-Saxon potential with fixed radius $R$ and diffuseness $a$ but whose depth is adjusted to give the appropriate binding energy. Their normalization was given by the corresponding spectroscopic factors [62]. The simplest configurations were adopted: $1 \mathrm{~s}_{1 / 2}^{2} 1 \mathrm{p}_{3 / 2}^{2}$ for the protons, $1 \mathrm{~s}_{1 / 2}^{2} 1 \mathrm{p}_{3 / 2}^{3}$ for the neutrons. The $1 \mathrm{~s}_{1 / 2}$ orbital was assumed to be bound by $26.5 \mathrm{MeV}$. The p-shell wave functions of Cohen and Kurath [136] indicate that the parentage for $1 \mathrm{p}$ proton pick-up from ${ }^{9} \mathrm{Be}$ is primarily the ground state of ${ }^{8} \mathrm{Li}$ with a few fragments at excitation energies up to a few $\mathrm{MeV}$. We represented this by the full strength at the centroid energy, corresponding to an average separation energy of $18 \mathrm{MeV}$. The parentage for $1 p$ neutron pick-up is predicted [136] to be distributed over a wide range of excitations in ${ }^{8} \mathrm{Be}$, but falls roughly into three groups corresponding to separation energy centroids of $1.67 \mathrm{MeV}, 5.1 \mathrm{MeV}$ and $17.9 \mathrm{MeV}$ with strengths of $0.58,0.72$ and 1.7 neutrons, respectively. Consequently, we used $1 \mathrm{p}_{3 / 2}$ orbitals with these energies and occupancies to construct the neutron density distribution.

As before, the correction due to center-of-mass recoil was handled in two ways. Method A used the harmonic oscillator approximation with the parameter $b=1.60 \mathrm{fm}$, corresponding to a correction of $-0.427 \mathrm{fm}^{2}$ to the mean square radius of the density distribution. Method $\mathrm{B}$ used effective binding energies $(A /(A-1))^{2}$ times the separation energies; this assures [62] the correct form for the tails of the form factors.

The shell-model potential well was chosen with $R=r_{0} \times 8^{1 / 3} \mathrm{fm}$ and $a=0.65 \mathrm{fm}$ and a spinorbit coupling of 17 times the Thomas term. The Coulomb field from a uniform charge of radius $2.7 \mathrm{fm}$ was included for the protons. The value of $r_{0}$ was adjusted until the proton distribution had an rms radius of $r_{\mathrm{p}}=2.39 \mathrm{fm}$, corresponding to the charge radius of $2.519 \mathrm{fm}$. In method A this led to $r_{0}=1.525 \mathrm{fm}$, while method B required $r_{0}=1.595 \mathrm{fm}$. The same potential wells were then used to generate the neutron distributions, except for adjustment of the depth to give the required binding. In this way, method A gave a neutron rms radius $r_{\mathrm{n}}=2.78 \mathrm{fm}$ so $r_{\mathrm{n}}-r_{\mathrm{p}}=0.395 \mathrm{fm}$, while method B gave $r_{\mathrm{n}}=2.77$ or $r_{\mathrm{n}}-r_{\mathrm{p}}=0.38 \mathrm{fm}$. The corresponding folded potentials for ${ }^{9} \mathrm{Be}+{ }^{40} \mathrm{Ca}$ near the strong absorption radius are given in table 5. Method $\mathrm{B}$ gives a slightly stronger potential but with a slightly steeper slope (smaller value of $\alpha$ ).

Variations on these prescriptions were tried to study the sensitivity of the results. First, if all the $1 \mathrm{p}$ neutrons were assumed to be bound by the separation energy $(1.67 \mathrm{MeV})$ of the odd neutron (cases $\mathrm{A}^{\prime}$ and $\mathrm{B}^{\prime}$ in table 5), $r_{\mathrm{n}}-r_{\mathrm{p}}$ increases to over $1.1 \mathrm{fm}$ and the folded potential doubles in strength at $r=9 \mathrm{fm}$ and has a much less steep slope $(\alpha=1 \mathrm{fm})$. On the other hand, removing the lp occupancy at $1.67 \mathrm{MeV}$ so that we just have 0.89 neutrons bound by $5.1 \mathrm{MeV}$ and 2.11 bound by $17.9 \mathrm{MeV}$ (case $\mathrm{A}^{\prime \prime}$ in table 5) reduces the folded potential by less than $20 \%$.

We also know that the "well-depth prescription" for the neutron form factors is not strictly 
Table 5

\begin{tabular}{lclll}
\multicolumn{5}{c}{ Various models of ${ }^{9} \mathrm{Be}$ and the ${ }^{9} \mathrm{Be}+{ }^{40} \mathrm{Ca}$ potential } \\
\hline Method $^{\text {a) }}$ & $\begin{array}{c}\left\langle r^{2}\right\rangle_{\mathrm{n}}^{1 / 2} \\
(\mathrm{fm})\end{array}$ & $\begin{array}{c}\left\langle r^{2}\right\rangle_{\mathrm{n}}^{1 / 2}-\left\langle r^{2}\right\rangle_{\mathbf{p}}^{1 / 2} \\
(\mathrm{fm})\end{array}$ & $\begin{array}{c}-\operatorname{Re} U(r=9) \\
(\mathrm{MeV})\end{array}$ & $\begin{array}{c}\alpha(r=9)^{\mathrm{b})} \\
(\mathrm{fm})\end{array}$ \\
\hline $\mathrm{A}$ & 2.78 & 0.395 & 1.11 & 0.76 \\
$\mathrm{~B}$ & 2.77 & 0.38 & 1.14 & 0.74 \\
$\mathrm{~A}^{\prime}$ & 3.62 & 1.19 & 2.27 & 1.005 \\
$\mathrm{~B}^{\prime}$ & 3.52 & 1.13 & 2.27 & 0.97 \\
$\mathrm{~A}^{\prime \prime}$ & 2.64 & 0.21 & 0.90 & 0.68 \\
$\mathrm{~B}^{\prime \prime}$ & 2.60 & 0.20 & 0.96 & 0.70 \\
\hline
\end{tabular}

a) See text.

b) Inverse of the logarithmic derivative of $\operatorname{Re} U$ at $r=9 \mathrm{fm}$.

correct [135]. To gain some idea of the error associated with this, we adopted what may be the opposite extreme (case $B^{\prime \prime}$ in table 5) of fixing the neutron well depth at $60 \mathrm{MeV}$, the value required in method B for the most tightly bound lp component, and reduced $r_{0}$ to give the correct binding for the two more weakly bound components; values of $r_{0}=1.1$ and $1.0 \mathrm{fm}$ were required. This procedure tends to reduce the radial extent of these two components. Indeed, $r_{n}-r_{p}$ is reduced to $0.2 \mathrm{fm}$, but the folded potential at $r=9 \mathrm{fm}$ is reduced only $15 \%$; it also becomes steeper $(\alpha=0.70 \mathrm{fm})$. The folded potentials for targets other than ${ }^{40} \mathrm{Ca}$ behave in a way very similar to those for ${ }^{40} \mathrm{Ca}$ given in table 5 .

\subsubsection{Analysis of ${ }^{9} \mathrm{Be}$ scattering}

The data available for ${ }^{9} \mathrm{Be}$ are somewhat limited. We considered measurements between 12 and $30 \mathrm{MeV}$ on ${ }^{28} \mathrm{Si},{ }^{40} \mathrm{Ca}$ and ${ }^{58} \mathrm{Ni}$ [137] and 121 and $201 \mathrm{MeV}$ on ${ }^{28} \mathrm{Si}$ [138]. There are also some data at $50 \mathrm{MeV}$ on ${ }^{28} \mathrm{Si},{ }^{40} \mathrm{Ca}$ and ${ }^{208} \mathrm{~Pb}[139]$, although their usefulness is reduced because the absolute normalization is uncertain to within about $\pm 15 \%$ and the energy is uncertain to about $\pm 1 \mathrm{MeV}$.

The value $r_{\mathrm{W}}=1.3 \mathrm{fm}$ was found satisfactory except for the highest energies on ${ }^{28} \mathrm{Si}$; these data demanded smaller values. The data for the various energies from Zurich [137] for each target were combined and fitted simultaneously. The optimum parameters are given in table 6 for potentials constructed according to method B; some of the fits have been shown elsewhere [133]. In every case a renormalization of $N \sim 0.5$ is required. The real potentials for ${ }^{9} \mathrm{Be}$ at the strong absorption radii $R=D_{1 / 2}$ are generally even weaker than found for ${ }^{6} \mathrm{Li}$ (table 4) and the imaginary potential in this region appears to be relatively stronger. We also note that the imaginary potential diffuseness required, $a_{\mathrm{W}} \approx 0.8 \mathrm{fm}$, is similar to that found for ${ }^{6} \mathrm{Li}$ but appreciably larger than that required for other projectiles. In both cases this is presumably related to the weaker binding energy of these nuclei which also results in the slope of the tail of the real potential being smaller (or $\alpha$ being larger) than for other nuclei.

These analyses provide strong evidence that the potential for ${ }^{9} \mathrm{Be}$ scattering is anomalous in the same sense as for ${ }^{6} \mathrm{Li}$. The model which accounts for the scattering of other projectiles predicts a potential which is about two times stronger than the empirical one, at least in the region near the strong absorption radius to which the scattering is sensitive. There is no clear evidence from the present results that this renormalization is dependent upon energy or target, although more extensive data will be required at the higher energies and for the heavier targets in order to be certain 


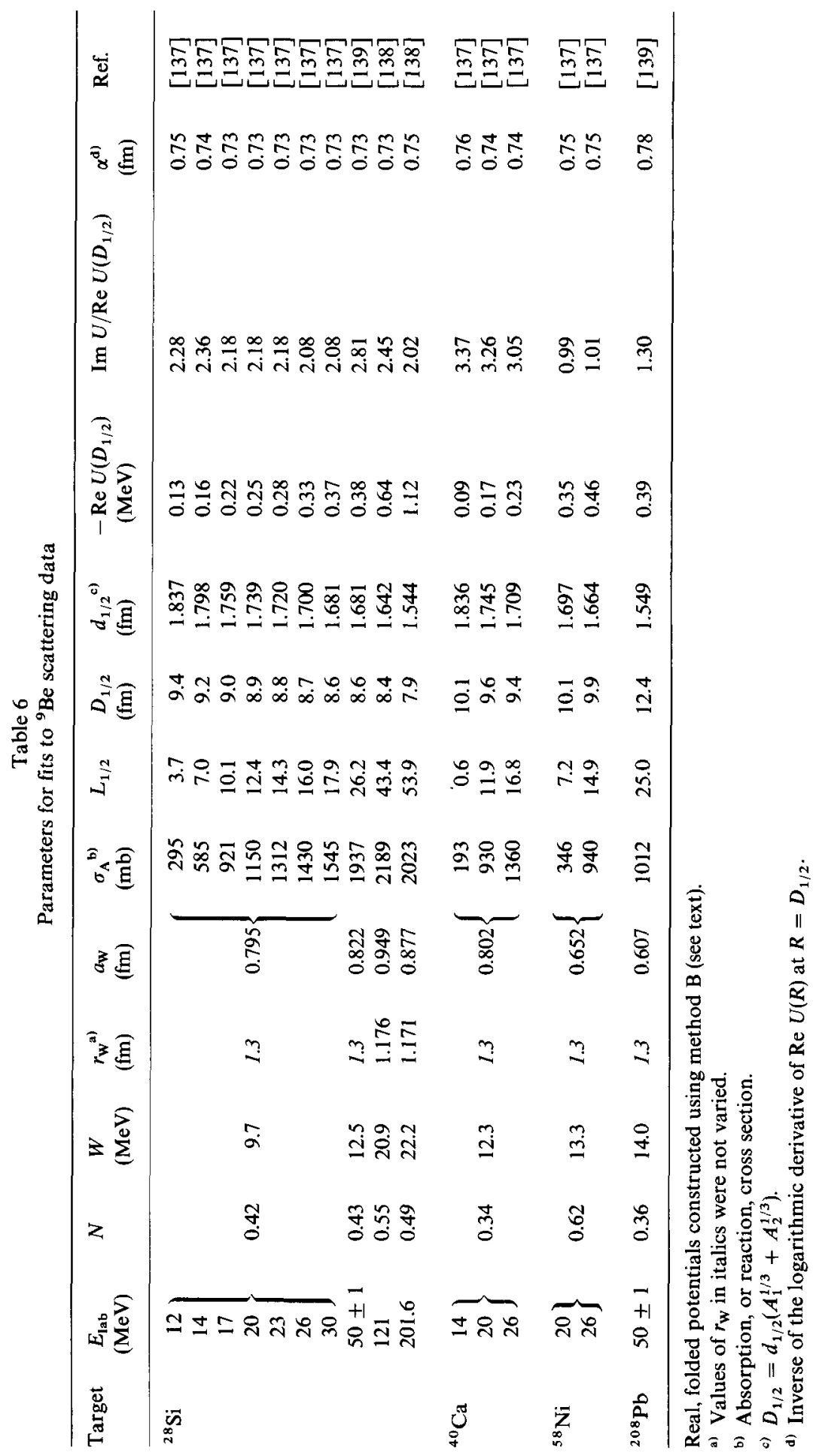


of this. Although, as table 5 indicates, there is some uncertainty in the value of $N$ due to the uncertainty in the neutron distribution in ${ }^{9} \mathrm{Be}$, this effect is much too small to account for $N \lesssim 0.5$. Two other effects omitted from the present analysis are those due to spin-orbit coupling and to scattering by the quadrupole moment of ${ }^{9} \mathrm{Be}$. Studies of the former with ${ }^{6} \mathrm{Li}$ indicated that it is negligible. The quadrupole scattering amplitude is incoherent with the main monopole amplitude and hence its effects are likely to be small, although it could be noticeable at larger angles, especially if the angular distribution had any oscillatory structure [92]. (We note that ${ }^{6} \mathrm{Li}$ differs from ${ }^{9} \mathrm{Be}$ in having a very small quadrupole moment.)

One feature that ${ }^{6} \mathrm{Li}$ and ${ }^{9} \mathrm{Be}$ share and which may be significant is that removal of a single nucleon leaves an unbound nucleus. The resulting continuum of 3-body states may play an important role in determining the higher-order corrections to the real folded potential.

\section{Inelastic scattering}

\subsection{Introduction}

The folding model may also be used to produce transition potentials $U_{\mathrm{tr}}(\boldsymbol{R})$ for inelastic scattering $[33,140]$. If only one of the nuclei is excited, the corresponding transition density $\rho_{\mathrm{tr}}(\boldsymbol{r})$ is inserted in the folding integral (5) in place of the ground-state density for that nucleus (see appendix B). The transition density may be obtained microscopically, such as from shell model or RPA particle-hole calculations [140], or macroscopically by deforming the ground-state density distribution $[33,35,72]$. Here we use the latter prescription. Just as for the elastic potential, particular care must be taken to include the transition density out to sufficiently large distances if the folding integration is to be done accurately. This is of concern especially if microscopic densities are to be used; structure calculations of this kind often do not use radial wavefunctions which are accurate at large distances (for example, they may use an oscillator basis which is quite adequate over the bulk of the nucleus but which is not extensive enough to give the tails of the wavefunctions accurately).

If the multipolarity of the transition is $L$, the folding integration then selects [33, 141] the $2^{L}$-pole component of the effective interaction $v\left(r_{12}\right)$ from the multipole expansion

$$
v\left(\left|\boldsymbol{r}_{1}-\boldsymbol{r}_{2}\right|\right)=\sum_{L} v_{L}\left(r_{1}, r_{2}\right) \sum_{M} Y_{L}^{M}\left(\boldsymbol{r}_{1}\right) Y_{L}^{M *}\left(\boldsymbol{r}_{2}\right)
$$

Only the term with $L=0$ contributes to the elastic scattering of spherical nuclei, so by studying inelastic transitions with $L>0$, we can probe the effective interaction $v$ in more detail. In particular, it has long been known that the relative cross sections for different multipoles depend sensitively upon the range of the interaction $[46,140,141]$. (Quadrupole, $L=2$, contributions to the predominantly $L=0$ elastic scattering from non-spherical nuclei have been considered previously [92] using the M3Y interaction.)

Two difficulties arise in applying these ideas. The first is that for low $L$-values, the sensitivity of the scattering to the range of the nuclear interaction is often reduced because of the dominance of the Coulomb excitation contributions (see [140] for example).

The second difficulty is due to uncertainties over the imaginary part of the effective interaction. We avoided this difficulty for elastic scattering by using a phenomenological Woods-Saxon imaginary potential. We could extend this hybrid approach to inelastic scattering by using folding for $\operatorname{Re} U_{\mathrm{tr}}(R)$ and the usual collective model approach for $\operatorname{Im} U_{\mathrm{tr}}(R)$ of deforming the Woods- 
Saxon imaginary potential obtained from a fit to the corresponding elastic scattering data. In this approach, $\operatorname{Im} U_{\mathrm{tr}}(\boldsymbol{R})$ for a given $L$ and to first order in the deformation is proportional to

$$
\beta_{L}^{(N)} R_{\mathrm{W}} \frac{\mathrm{d}}{\mathrm{d} r}[\operatorname{Im} U(R)]=\beta_{L}^{(N)}\left(\frac{W R_{\mathrm{W}}}{a_{\mathrm{W}}}\right) \frac{\mathrm{e}^{x}}{\left(\mathrm{e}^{x}+1\right)^{2}},
$$

where $\operatorname{Im} U$ and $x$ are defined by eq. (26). Thus, its shape is independent of $L$. We then have to adopt some prescription for the value of the deformation parameter $\beta_{L}^{(N)}$. Further, it is not clear that the correct shape is given by eq. (32) with parameters $R_{\mathrm{W}}$ and $a_{\mathrm{W}}$ taken from elastic scattering. It is well known $[33,44,141-143]$ that the result of the corresponding prescription for $\operatorname{Re} U_{\mathrm{tr}}(\boldsymbol{R})$ may be quite different in shape from the ( $L$-dependent) shapes obtained in the folding model. (We discuss further examples below in which the deformation and folding models give very different overall shapes for $\operatorname{Re} U_{\mathrm{tr}}$. However, in these cases, the two prescriptions do give almost the same magnitudes and similar slopes for $\operatorname{Re} U_{\mathrm{tr}}$ in the vicinity of the strong absorption radius provided the corresponding optical potentials give the same elastic scattering and the appropriate deformation parameters are used.)

These difficulties are avoided formally by assuming the imaginary interaction to have the same shape as the real one. This corresponds to using a complex $N$ factor for elastic scattering. When this is adequate for fitting elastic data, the same complex $N$ may be used in a consistent analysis of the inelastic scattering without introducing further parameters. Unfortunately, the physics of this procedure is questionable. At the very least, we expect $[5,144]$ the imaginary part of the effective interaction to be strongly density dependent. The procedure may be acceptable if both elastic and inelastic scattering are only sensitive to the potential near the strong absorption radius and if the real and imaginary parts of the effective interaction have similar ranges.

Uncertainties in the imaginary interaction for inelastic scattering are of particular importance when there is significant interference between the amplitude due to the nuclear interaction and that due to Coulomb excitation. This interference is sensitive to the relative phase of the two amplitudes [145]. (Examples of differences in the results of using the two prescriptions for $\operatorname{Im} U_{\mathrm{tr}}$ just discussed are shown in [140].)

Despite these various uncertainties, good fits to data for the inelastic scattering of heavy ions have been obtained in analyses which use the folding model for both the elastic and inelastic scattering in a consistent way. Most of these have used a semi-phenomenological effective interaction of Gaussian form with a "reasonable" range (for example, ranges of $1 \mathrm{fm}[44,140]$ or $1.4 \mathrm{fm}$ $[33,143])$. The range of the M3Y interaction used here is predetermined and not adjustable. It has been applied to a few cases of heavy ion inelastic scattering which we now discuss.

\subsection{Applications}

\subsubsection{Procedure}

The transition potential $U_{\mathrm{tr}}(\boldsymbol{R})$ constructed using the folding model may, of course, be used in coupled-channel calculations. This was not done for the cases considered here; instead the distorted-wave approximation (DWA) $[46,146]$ was used. One reason is that a coupled-channel approach would have required reanalysis of the elastic data for consistency and this is timeconsuming and costly. Consequently, we might expect only qualitative agreement with the data (see [147] for example). Certainly the DWA is not adequate for excitation of the $4{ }^{+}$state in ${ }^{28} \mathrm{Si}$ or for the mutual excitation of two ${ }^{12} \mathrm{C}$ ions, both of which receive strong two-step contributions. 
Only quadrupole $L=2$ excitations are considered here and in each case the form of the Tassie model [148] was used for the transition densities (appendix B),

$$
\rho_{\mathrm{tr}}(r)=\frac{C}{\sqrt{5}} r \frac{\mathrm{d} f(r)}{\mathrm{d} r}, \quad f(r)=\left[1+\exp \left(\frac{r-c}{d}\right)\right]^{-1} .
$$

In the strict hydrodynamical model, the radius $c$ and diffuseness $d$ would be the same as those for the 2-parameter Fermi shape which describes the ground state density distribution. In practical analyses of inelastic electron scattering data, this constraint is often relaxed. When possible, we should use the parameters obtained from electron scattering, with correction for the finite size of the proton (and neutron) charge distribution. For the predominantly isoscalar transitions considered here, we assume that the contributions from the neutron and proton excitations have the same shape with magnitudes in the ratio $N / Z$. The constant $C$ was chosen so that the proton part of $\rho_{\mathrm{tr}}(r)$ would yield the measured $B(\mathrm{E} 2)$ value, where [149]

$$
B(\mathrm{E} 2) \uparrow=5\left|\int \rho_{\mathrm{tr}}(r) r^{4} \mathrm{~d} r\right|^{2} e^{2} \approx\left[\left(\frac{Z C}{A}\right)\left(c^{5}+\frac{10}{3} \pi^{2} d^{2} c^{3}+\frac{7}{3} \pi^{4} d^{4} c+120 d^{5} \exp \left(-\frac{c}{d}\right)\right)\right]^{2} e^{2}
$$

As just discussed, the imaginary part of $U_{\mathrm{tr}}(\boldsymbol{R})$ was either chosen to have the same shape as the folded, real part (with a complex $N$ obtained from the elastic data) or by deforming the WoodsSaxon imaginary potential obtained from the elastic fit according to eq. (32). For the latter approach, the nuclear potential deformation parameter $\beta_{2}^{(N)}$ was determined from the relation

$$
\beta_{2}^{(N)} R_{\mathrm{W}}=\beta_{2}^{(\mathrm{c})} R_{\mathrm{c}} \text {. }
$$

Here $\beta_{2}^{(\mathrm{c})}$ is the charge deformation parameter extracted in the usual way from the measured $B(\mathrm{E} 2)$ using a uniform charge radius of $R_{\mathrm{c}}=1.2 A^{1 / 3} \mathrm{fm}$.

The contribution from Coulomb excitation was included in the usual way, again using the measured $B(\mathrm{E} 2)$ value. Consequently, once the $B(\mathrm{E} 2)$ is known, this procedure for constructing the transition potential has no free parameters and allows us to make an absolute comparison of the predictions of the M3Y interaction with inelastic data. The optical potentials used in the DWA were the corresponding ones which fit the elastic data.

\subsubsection{Excitation of ${ }^{60} \mathrm{Ni} b y{ }^{16} \mathrm{O}$}

The excitation of the $1.33-\mathrm{MeV} 2^{+}$state by electrons [150] led to $B(\mathrm{E} 2)=(918 \pm 26) e^{2} \mathrm{fm}^{4}$, corresponding to $\beta_{2}^{(\mathrm{c})}=0.205 \pm 0.003$. Analysis of the electron measurements also gave $c=4.25 \mathrm{fm}$, $d=0.518 \mathrm{fm}$ for the transition charge density. To account for the finite size of the proton charge, we simply reduced the diffuseness to $d=0.47 \mathrm{fm}$; this reduces the transition radius by the required amount $[151]$. Then we need $C=0.0326 \mathrm{fm}^{-3}$.

The radial parts of the transition density and potential for ${ }^{16} \mathrm{O}+{ }^{60} \mathrm{Ni}$ are shown in fig. 20 , together with the real part of the transition potential obtained by deforming a Woods-Saxon potential which fits the elastic data [35]. (The latter was normalized using eq. (34) with the real potential radius instead of $R_{\mathrm{W}}$.) Although the folded and deformed potentials are very different for $R \lesssim 8 \mathrm{fm}$, they are almost the same in both magnitude and slope in the vicinity of the strong absorption radius $(R \sim 9-10 \mathrm{fm})$. The corresponding inelastic cross sections were compared in [35] with the measurements taken at $142 \mathrm{MeV}$; fig. 21 shows a comparison with 61-MeV data. 


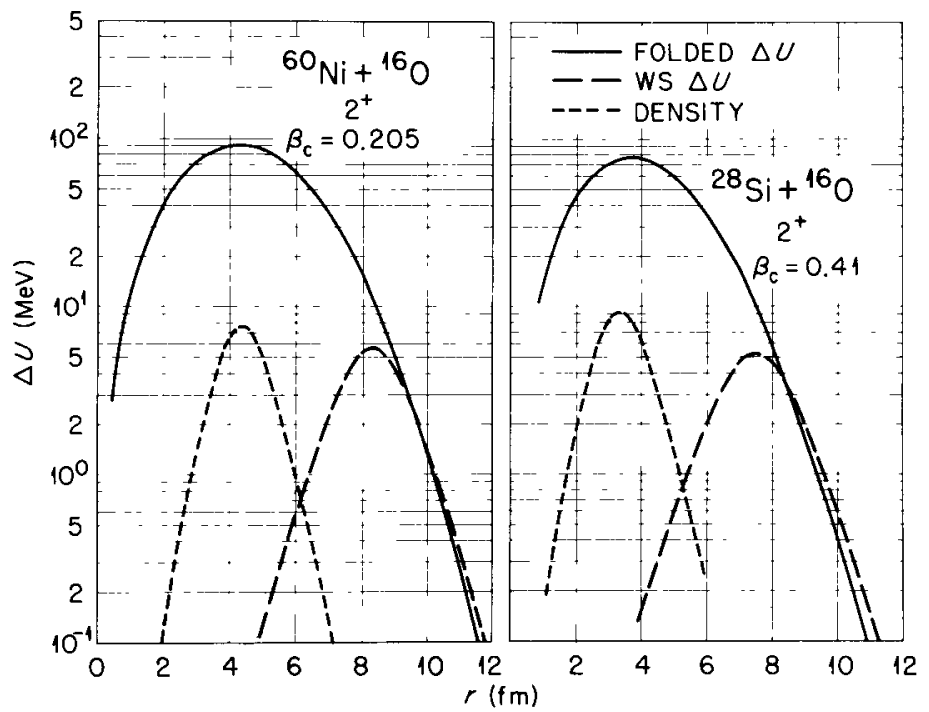

Fig. 20. Transition potentials and densities for inelastic excitation of the lowest $2^{+}$states of ${ }^{28} \mathrm{Si}$ and ${ }^{60} \mathrm{Ni}$. The radial part of the transition potential is denoted $\Delta U$. The curves with long dashes were obtained by deforming to first order Woods-Saxon (WS) potentials which fitted the corresponding elastic scattering data [35].

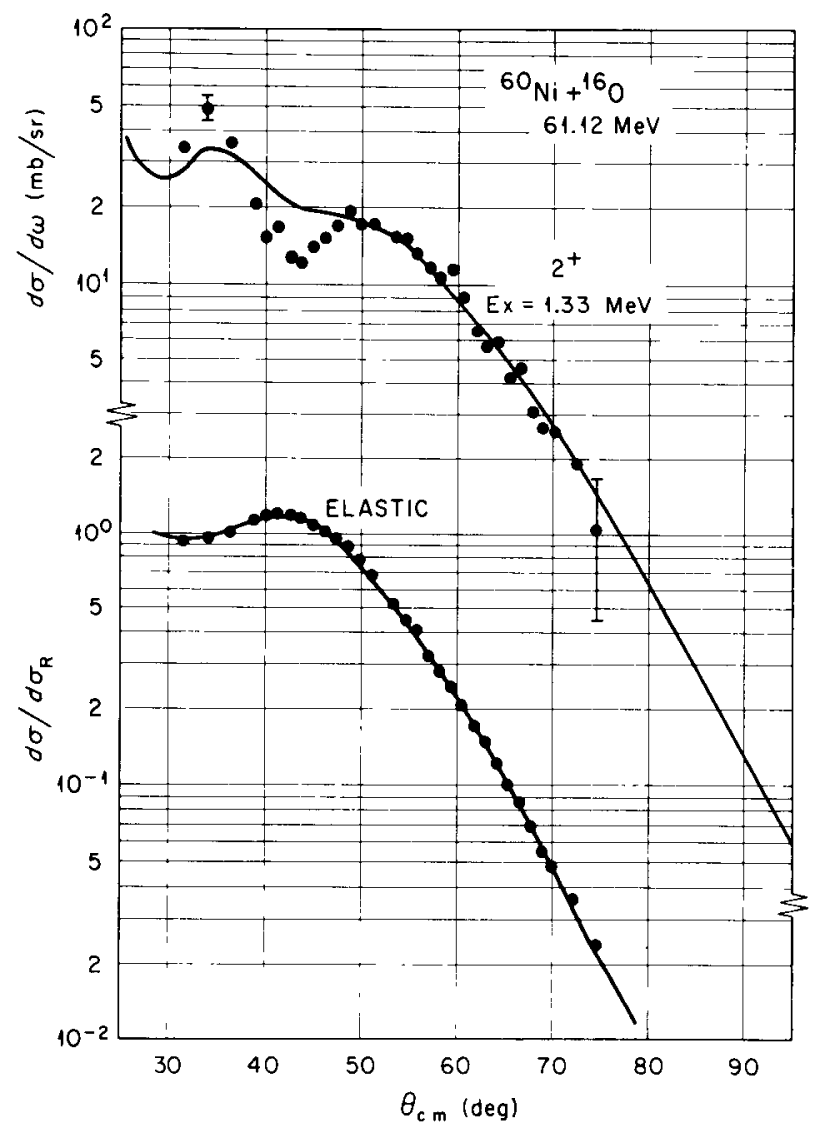

Fig. 21. Comparison with the data of the inelastic scattering for ${ }^{16} \mathrm{O}+{ }^{60} \mathrm{Ni}$ at $61 \mathrm{MeV}$ predicted by the folding model. 
In both cases the agreement is good in both magnitude and shape, except for some deviations at the small angles where interference between nuclear and Coulomb excitation is important. These deviations may be due to higher-order effects not included in the DWBA or to the imaginary part of the transition potential being incorrect. (In this case, the results of using either a complex folded potential or a Woods-Saxon imaginary part are indistinguishable [35] at $61 \mathrm{MeV}$ and only differ by a few per cent at $142 \mathrm{MeV}$, even in the interference region.)

\subsubsection{Excitation of ${ }^{28} \mathrm{Si}$ by ${ }^{16} \mathrm{O}$}

There is some uncertainty in the $B(\mathrm{E} 2)$ value for the excitation of the $2^{+}$state at $1.77 \mathrm{MeV}$ in ${ }^{28} \mathrm{Si}$. We adopted the average value [152] of $B(\mathrm{E} 2)=327 e^{2} \mathrm{fm}^{4}$, corresponding to $\beta_{2}^{(\mathrm{c})}=0.41$. We also chose transition density parameters $c=3.2 \mathrm{fm}$ and $d=0.5 \mathrm{fm}$ so that $C=0.0556 \mathrm{fm}^{-3}$. These are representative of the $c$ and $d$ parameters needed to describe the ground-state density distribution, and we have found that the predicted inelastic scattering is not very sensitive to small variations in these parameters provided the same $B(E 2)$ value is reproduced. (More recent measurements [153] have led to $B(\mathrm{E} 2)=337 \pm 30 e^{2} \mathrm{fm}^{4}$, with $c=2.68 \mathrm{fm}$ and $d=0.536 \mathrm{fm}$ for the transition charge density.) The potential and density for ${ }^{16} \mathrm{O}+{ }^{28} \mathrm{Si}$ are also shown in fig. 20, together with the real part of a deformed Woods-Saxon potential which fits the elastic data [35] and normalized using eq. (34) with the real potential radius instead of $R_{\mathrm{W}}$. Again the folded and deformed transition potentials are very different in the interior but similar in the surface. It was shown in [35] that these potentials resulted in reasonable fits to inelastic data at $141.5 \mathrm{MeV}$. Figure 22 shows a similar comparison for scattering at $37.7 \mathrm{MeV}$. In this case the "global" folded

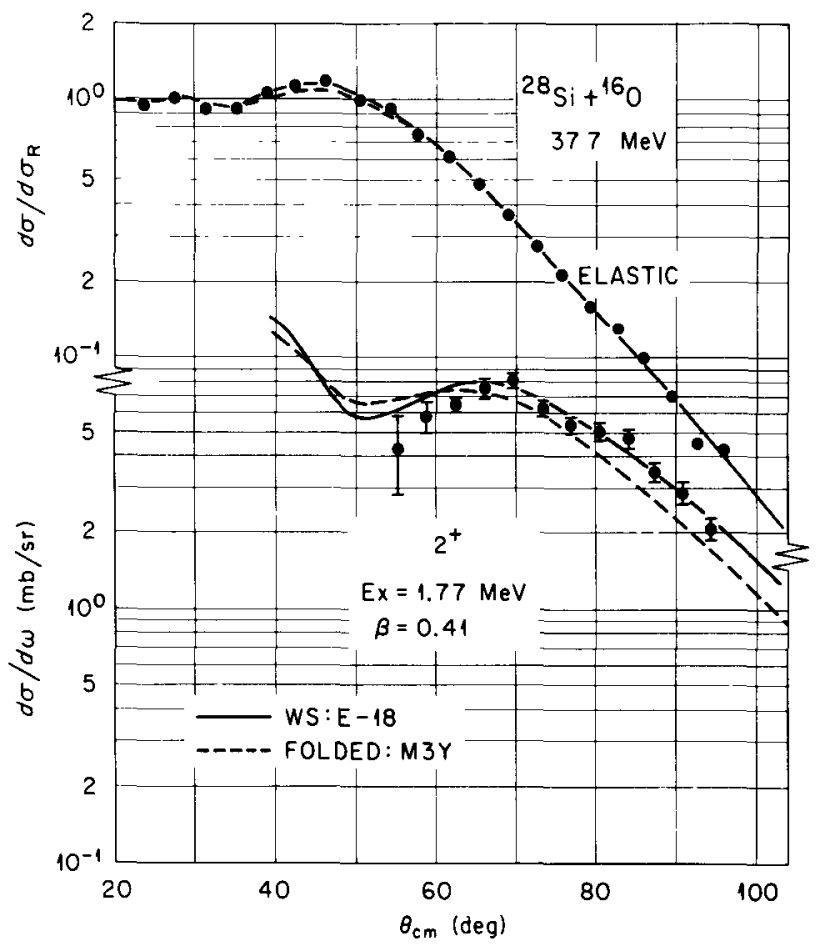

Fig. 22. Comparison with the data of the inelastic scattering for ${ }^{16} \mathrm{O}+{ }^{28} \mathrm{Si}$ at $38 \mathrm{MeV}$ predicted by the global folding model of [94] (solid curve) and the deformed Woods-Saxon (WS) potential model (dashed curve; the global potential E-18 of [115] was used). 
potential of [94] and the "global" Woods-Saxon form of [115] were used. The folded model agrees less well with the data, although the agreement is quite good considering that these are absolute predictions. If we adjust the folded optical potential renormalization factor $N$ (to $N \approx 1.0$ ) and the parameters of the associated imaginary Woods-Saxon potential to optimize the fit to the particular elastic data at $37.7 \mathrm{MeV}$, much better agreement with the inelastic scattering is obtained. The discrepancies seen in fig. 22 are due to inadequacies of the potentials used for the elastic channels, rather than the M3Y interaction itself.

\subsubsection{Single excitation of ${ }^{12} \mathrm{C}+{ }^{12} \mathrm{C}$}

Differential cross sections for the excitation of one nucleus to its $2^{+}$state at $4.44 \mathrm{MeV}$ in the scattering of ${ }^{12} \mathrm{C}+{ }^{12} \mathrm{C}$ were measured for 14 energies between 70 and $127 \mathrm{MeV}$ [72]. The transition charge density for this excitation has not been well-determined experimentally. We chose to use $B(\mathrm{E} 2)=42 e^{2} \mathrm{fm}^{4}[154]$. (More recent measurements [155] give $39 \pm 4 e^{2} \mathrm{fm}^{4}$.) In the choice of $c$ and $d$, we were guided by analyses of electron scattering data [156]; the transition density obtained in that way is quite well reproduced by using $c=1.5 \mathrm{fm}, d=0.65 \mathrm{fm}$ in eq. (33) with $C=0.171 \mathrm{fm}^{-3}$. In order to test the sensitivity of the scattering to the details of the density, we also made calculations with $c=2.5 \mathrm{fm}, d=0.50 \mathrm{fm}\left(C=0.099 \mathrm{fm}^{-3}\right)$ and with $c=1.5 \mathrm{fm}$, $d=0.50 \mathrm{fm}\left(C=0.456 \mathrm{fm}^{-3}\right)$. Figure 23 shows these densities and the corresponding folded transition potentials. Although the densities are very different, the potentials are quite similar, especially near $R \approx 6 \mathrm{fm}$ which is just inside the strong absorption radius for ${ }^{12} \mathrm{C}+{ }^{12} \mathrm{C}$ scattering (table 3 ). In particular, the full and dashed curves are very close and indeed we find that they yield almost identical inelastic cross sections.

The $B(\mathrm{E} 2)$ value we use corresponds to $\beta_{2}^{(\mathrm{c})}=0.60$ and this was used, with eq. (34), to deform the Woods-Saxon imaginary potential (table 3). Including this imaginary part of the transition potential increases the predicted inelastic cross sections by 30 to $40 \%$ in magnitude, with some small changes in the angular distributions, and improves the agreement with the data. Coulomb excitation was found to change the cross sections by at most a few per cent.

Comparisons with the data at a representative set of energies are shown in fig. 24 for the parameters $c=1.5 \mathrm{fm}, d=0.65 \mathrm{fm}$. The agreement in magnitude is very good and the predicted shapes are also very satisfactory considering that (i) no adjustable parameters were available, and (ii) the DWA was used. Further, some of the details of the angular distributions are quite sensitive to small changes in the parameters (just as is the case with the elastic scattering). For example, the angular distribution at the lowest energy of $70.7 \mathrm{MeV}$ oscillates somewhat out of phase with the data but a $5 \%$ increase in the real potential (from $N=1.01$ to $N=1.06$ ) changes this drastically (fig. 24). This result is due to the changes produced in the elastic distorted waves, rather than in the transition potential.

There are small fluctuations in the optimum values of $W$ at the various energies [72] so the sensitivity of the inelastic scattering to these was studied. The general characteristic of increasing $W$ is to reduce the cross section at the larger angles as indicated by the dotted curve in fig. 24 for 93.8 $\mathrm{MeV}$.

\subsection{Discussion}

In summary, a remarkably good parameter-free account of inelastic scattering is obtained if we use the M3Y interaction folded with a transition density suggested by electron scattering measure- 


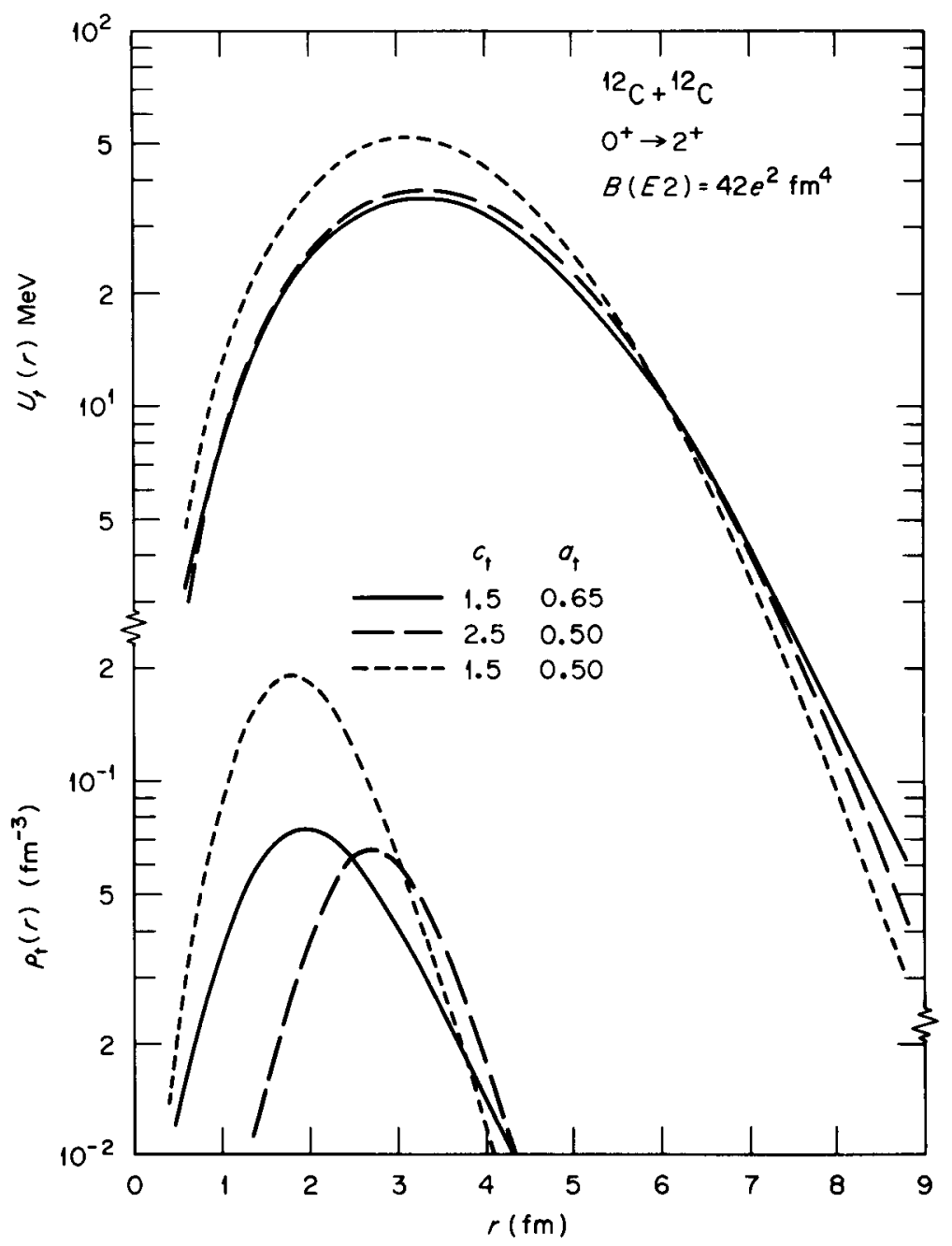

Fig. 23. Folded transition potentials $U_{\mathrm{t}}$ for single-excitation of the $4.44-\mathrm{MeV} 2^{+}$state in ${ }^{12} \mathrm{C}$ for various choices of transition density $\rho_{\mathrm{t}}$.

ments and, especially, normalized to give the corresponding observed $B(\mathrm{E} 2)$ value. We further assumed that the neutron transition density was $(N / Z)$ times the proton one and included an imaginary transition potential obtained by deforming (according to eqs. (32) and (34)) the WoodsSaxon imaginary potential required to fit the elastic data. The small discrepancies remaining could well be due to inadequacies in the imaginary potential and in the use of the DWA.

Hence we conclude tentatively that the M3Y interaction can be used successfully to describe inelastic as well as elastic heavy ion scattering. More detailed conclusions must await studies of other nuclei, as well as examination of coupled-channel effects and a better understanding of the imaginary potential. 


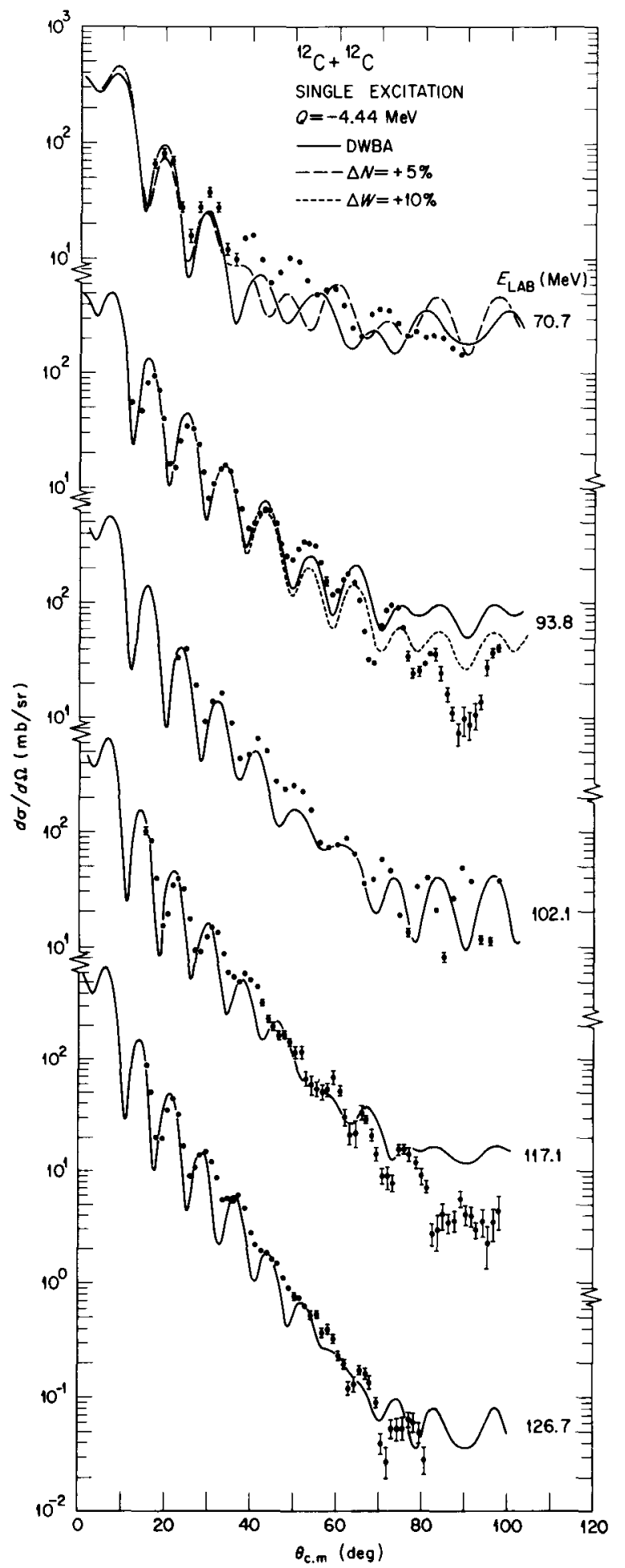

Fig. 24. Comparison with the data at representative energies of the inelastic scattering of ${ }^{12} \mathrm{C}+{ }^{12} \mathrm{C}$ predicted by the folding model. The dashed curve at $70.7 \mathrm{MeV}$ shows the effect of increasing the strength of the real interaction by $5 \%$ in both the elastic and inelastic channels. The dotted curve at $93.8 \mathrm{MeV}$ shows the effect of increasing the absorptive strength by $10 \%$. 


\section{Summary and discussion}

Although phenomenological optical potentials are often used to describe the elastic scattering of heavy ions, the use of calculated potentials is appealing because such potentials require as input information either from other nuclear processes such as nucleon-nucleon and electron-nucleus scattering or from nuclear models which may thereby be checked. Further such calculations allow one to predict the potentials for systems for which elastic scattering data are not available. We have taken a step in this direction for the real part of the optical potential by using the doublefolding model approximation.

We have chosen to use the definition (3) of the optical potential for heavy-ion scattering which is given by Feshbach's projection operator formalism. This governs the $\chi_{00}(R)$ term of the expansion (2) of the total wavefunction, which describes the relative motion of the two nuclei while they both remain in their ground states. This potential consists of a real, folded term $U_{\mathrm{F}}$ plus a complex remainder $\Delta U$. We have studied the validity of using $U_{\mathrm{F}}$ alone for the real part of the optical potential by comparison with experimental scattering data. This implies that $\operatorname{Re} \Delta U \ll U_{\mathrm{F}}$ and can be neglected, at least in the region to which the scattering is sensitive. A phenomenological, Woods-Saxon form was used to represent $\operatorname{Im} \Delta U$. Effective nucleon-nucleon interactions based upon realistic $G$-matrices were used to construct the folded potential $U_{\mathrm{F}}$. In principle, the formula for $U_{F}$ should take complete account of the exchange of nucleons between the two nuclei due to antisymmetrization. We have chosen to include only the "knock-on" single nucleon exchange (SNE); that is, as implied by eq. (14), the exchange of the two interacting nucleons. This was done on the grounds that all the other exchange terms require the interchange between projectile and target of one or more pairs of nucleons without the presence of an interaction between them. While these can occur, the overlaps involved in the folding integral tend to be smaller, especially for the important peripheral collisions [54]. Further, the SNE term retained was treated approximately by use of the pseudopotential (15).

In an earlier review [37] it had been concluded that double-folding overestimated the real potential; however, it is now known [48] that this was due to an improper choice for the representation of the effective nucleon-nucleon interaction, especially of its range. In particular, the longrange OPEP component was sometimes treated incorrectly. The present work shows that the double-folding model with a properly chosen effective interaction, in particular the M3Y interaction [11], can reproduce the scattering of many systems with bombarding energies in the range of 5 to $20 \mathrm{MeV}$ per nucleon. The real potentials for these systems are given correctly if the calculated folded potentials are renormalized by a factor $N$ where $N \approx 1.11 \pm 0.13$ (see table 3). The deviation of $+11 \%$ of the average $N$ from unity might be due to an underestimate of the SNE when using the zero-range approximation (15), or it may reflect some other small deficiency such as the neglect of $\operatorname{Re} \Delta U$ or the other exchange terms. Very similar results are given by the density-dependent DDD interaction [50]. Preliminary applications to inelastic scattering appear to be equally successful. The same interactions are also successful in describing the few cases of alpha scattering studied so far. They are moderately successful when applied to nucleon scattering, although they appear to predict real potentials with mean square radii which are slightly too small; this may also be due in part, at least, to the use of the zero-range approximation to SNE. Although the density-dependent DDD interaction gives very similar results for nucleons as well, it is also possible that other forms of density-dependence may explain the discrepancies [82].

The only exception established so far occurs for the scattering of ${ }^{6} \mathrm{Li}$ and ${ }^{9} \mathrm{Be}$ which require a 
reduction in the strength of the calculated folded potential by a factor of about two. The reason for this is not known at present, but presumably it is associated with the weak binding of these two nuclei. Preliminary results of analyses of the scattering of other non-closed-shell nuclei, such as ${ }^{13} \mathrm{C},{ }^{14,15} \mathrm{~N}$ and ${ }^{17,18} \mathrm{O}$, indicate that these are not "anomalous" and that folded potentials with $N \approx 1$ can give agreement with the data [162].

The magnitude of the SNE terms depends upon the choice for the odd-state effective interactions. However, these terms are important because, although the SNE contribution from the even-state interactions is small $(\sim 10 \%)$, the exchange contribution from the non-OPEP part of the odd-state forces almost exactly cancels the direct contribution (see table 2). Consequently, the inclusion of SNE greatly reduces the sensitivity of the results to the choice of the poorly-known odd-state forces.

Most of the scattering data we have examined is sensitive only to the potential at large separations, perhaps a favorable circumstance for the folding model to be successful. However, there are a few cases, associated with absorption which is weaker than usual, where the scattering is sensitive to the interior of the potential. The model was found to be successful for some of these, namely $\alpha+\alpha, \alpha+{ }^{40} \mathrm{Ca}$ and ${ }^{12} \mathrm{C}+{ }^{12} \mathrm{C}$. This implies that the very deep potentials predicted by our model are indeed appropriate, although it is possible [27], at least for ${ }^{12} \mathrm{C}+{ }^{12} \mathrm{C}$, that discrete ambiguities exist that would allow potentials to be used that are somewhat more shallow in the interior provided their values in the surface were unchanged. (For ${ }^{12} \mathrm{C}+{ }^{12} \mathrm{C}$, for example, "surface" here means $R \gtrsim 4 \mathrm{fm}$ [72].) However, it does seem very unlikely that this could be so for the $\alpha+\alpha$ scattering. It would indeed be a cruel jest of nature if the good agreement obtained with the M3Y interaction in these cases should eventually be shown to be mere accident!

Preliminary studies suggest that the folding model may also be able to reproduce the "anomalous" large-angle scattering observed $[100,105]$ for other systems such as ${ }^{16} \mathrm{O}+{ }^{28} \mathrm{Si},{ }^{12} \mathrm{C}+{ }^{40} \mathrm{Ca}$ and ${ }^{16} \mathrm{O}+{ }^{40} \mathrm{Ca}$. Even ${ }^{6} \mathrm{Li}+{ }^{40} \mathrm{Ca}$ at $30 \mathrm{MeV}$ is fitted (see fig. 19) with a simple radially-independent renormalization of $N \approx 0.6$, although this scattering is affected by the potential for separations as small as $R \approx 3 \mathrm{fm}$.

When the validity of the folding model with a given interaction has been established, it may then be used to obtain information from heavy-ion scattering on the matter distributions in nuclei. In particular, it may provide further evidence on differences between neutron and proton distributions, as was indicated for example by the discussion above on ${ }^{12} \mathrm{C}+{ }^{142} \mathrm{Nd}$. (Of course, this approach has been used for some time in the analysis of elastic alpha scattering; see $[3,86]$ for example.) The estimate presented in appendix B implies that a change of $0.1 \mathrm{fm}$ in the neutron RMS radius in a typical case would result in a change in the folded potential of order $20 \%$; this is in agreement with the results for ${ }^{12} \mathrm{C}+{ }^{142} \mathrm{Nd}$.

The success of the folding model used here, if not accidental, implies that the term $\operatorname{Re} \Delta U$ in eq. (3) is small compared to $U_{\mathrm{F}}(R)$ in the region of $R$ that is important. The results of Vinh Mau [23] for $\alpha+{ }^{40} \mathrm{Ca}$ suggest that indeed this is the case. The work of Love et al. [24] indicates that Vinh Mau's result was obtained because of her assumption that the important inelastic transitions are to states with excitation energies much smaller than the bombarding energy. This implies that the associated momentum transfers $q$ are predominantly much smaller than the momentum $k$ of relative motion; then the factor $[1+\mathrm{i}(\pi k / q)]$ in eq. (20) of Love et al. [24] leads to $\operatorname{Re} \Delta U \ll \operatorname{Im} \Delta U$. The work of [24] was concerned with the contributions to $\Delta U$ from Coulomb excitation for which the assumption $q \ll k$ is a good one. That this assumption might be reasonable even for the short-ranged nuclear interactions is suggested by the representation of the matrix elements in momentum space given in appendix B; for example, eqs. (B.3) and (B.9). Although 
the interaction $v$ has a short range and hence $\tilde{v}$ contains high momentum components, it is always associated with the density $\rho$ of the ion responsible for the excitation. The relatively large size of $\rho$ tends to prevent the high momentum components of $v$ from contributing. In other words, the interaction responsible for the excitation of one nucleus is not $v$ itself but $v$ folded into $\rho$ for the other nucleus; this is a relatively long-ranged object typical of a nucleon-nucleus optical potential. However, we must await explicit calculations to see whether this argument is sufficient to make $\operatorname{Re} \Delta U$ negligible.

For the future, it will be valuable to give more attention to cases like those exhibiting "anomalous" large-angle scattering because these may reveal more about the potential at smaller distances. It is important to confirm whether the folding model remains valid here or, if not, how it has to be modified. This should include studies of excitation functions as well as angular distributions and may involve the introduction of modified imaginary potentials (such as the use of $L$-dependence or surface transparency).

A more sophisticated interaction should also be used. The energy-dependence $[5,6]$ should be introduced explicitly along with the density-dependence. Further, it is desirable to have a $G$-matrix which is more appropriate to heavy-ion scattering, in which the two interacting nucleons are immersed in two pieces of nuclear matter which are moving with respect to each other [144], especially when the energy per nucleon becomes comparable to the Fermi energy. For considerably higher energies ( $\gtrsim 100 \mathrm{MeV}$ per nucleon) we may anticipate the use of the free nucleon-nucleon $t$-matrix as a complex effective interaction; a representation of this similar to that used here for the $G$-matrix has already been applied successfully to nucleon scattering [157]. Finally, it would be much more satisfactory if we could also base the imaginary potential upon microscopic calculations (see [144] for example) rather than treat it phenomenologically as was done here.

\section{Acknowledgments}

We are indebted to B. Day for providing us with numerical values of his G-matrix and to J.W. Negele for giving us his density distributions. We thank F.G. Perey and L.W. Owen for the use of the optical model code HIGENOA and L.D. Rickertsen for the folding code DOLFIN. We wish also to thank the many experimentalists who have sent us their data in tabular form, sometimes before publication.

\section{Appendix A: Spin and isospin effects in folded potentials}

An interaction in the form of eq. (6) can give rise, in some circumstances, to both a spin- and an isospin-dependent folded potential. Here we show that these terms are usually quite small.

Consider isospin first and two spherical even-even nuclei, each with the spins of their constituent nucleons paired off. We can rewrite eq. (5) in a form which better displays the role of isospin as

$$
U_{\mathrm{F}}(R)=\int \mathrm{d} \boldsymbol{r}_{1} \int \mathrm{d} \boldsymbol{r}_{2}\left[\rho_{1 \mathrm{p}} \rho_{2 \mathrm{p}} v_{\mathrm{pp}}+\rho_{1 \mathrm{p}} \rho_{2 \mathrm{n}} v_{\mathrm{pn}}+\rho_{1 \mathrm{n}} \rho_{2 \mathrm{n}} v_{\mathrm{nn}}+\rho_{1 \mathrm{n}} \rho_{2 \mathrm{p}} v_{\mathrm{pn}}\right]
$$

where, for example, $\rho_{i \mathrm{p}}$ is the proton point-density (as a function of $\boldsymbol{r}_{i}$ ) of nucleus $\mathrm{A}_{i}$ and $v_{\mathrm{pp}}$ is 
the spin-independent part of the proton-proton interaction. In terms of eq. (6)

$$
\begin{aligned}
v_{\mathrm{pp}}=v_{\mathrm{nn}} & =v_{00}+v_{01} \\
v_{\mathrm{pn}} & =v_{00}-v_{01}
\end{aligned}
$$

and

$$
\rho_{i}=\rho_{i \mathrm{p}}+\rho_{\text {in }}
$$

Using these relations, $U_{\mathrm{F}}$ can be re-expressed as

$$
U_{\mathrm{F}}(R)=\int \mathrm{d} r_{1} \int \mathrm{d} r_{2}\left[v_{00} \rho_{1} \rho_{2}+v_{01}\left(\rho_{1 \mathrm{n}}-\rho_{1 \mathrm{p}}\right)\left(\rho_{2 \mathrm{n}}-\rho_{2 \mathrm{p}}\right)\right]
$$

If we tentatively assume $\rho_{i \mathrm{n}}=\left(N_{i} / A_{i}\right) \rho_{i}, \rho_{i \mathrm{p}}=\left(Z_{i} / A_{i}\right) \rho_{i}$, we find

$$
U_{\mathrm{F}}(R)=\int \mathrm{d} r_{1} \int \mathrm{d} r_{2} \rho_{1} \rho_{2}\left[v_{00}+v_{01} \frac{\left(N_{1}-Z_{1}\right)\left(N_{2}-Z_{2}\right)}{A_{1} A_{2}}\right] \rightarrow U_{0}+\frac{4 U_{1} \boldsymbol{T}_{1} \cdot \boldsymbol{T}_{2}}{A_{1} A_{2}} .
$$

Although the assumption $\rho_{\mathrm{n}}=(N / Z) \rho_{\mathrm{p}}$ is certainly suspect, it does provide a rough estimate of the isospin-dependent term. For most colliding systems, the coefficient of $v_{01}$ is small. For example, in the case of ${ }^{13} \mathrm{C}+{ }^{207} \mathrm{~Pb}$, the term in square brackets becomes $v_{00}+0.016 v_{01}$. From eq. (22) most realistic interactions satisfy $v_{01} / v_{00} \sim-0.5$ with the result that the isospin-dependent term is $\sim 1 \%$ of the $v_{00}$ term and typically has the opposite sign. An explicit calculation using the interaction in eqs. (10) and (21) has been made for this system. The isospin-dependent term is found to reduce the magnitude of $U_{\mathrm{F}}(R)$ at the strong absorption radius, $R \approx 12.3 \mathrm{fm}$, by $\sim 2 \%$ which is negligible, considering the other uncertainties. The $v_{01}$ term allows the possibility of chargeexchange reactions also; of course, $v_{00}$ does not contribute to these. However, the estimate just presented does imply that the cross section for such reactions should be quite small.

For elastic scattering, the spin-dependent parts $\left(\sim \sigma_{1} \cdot \sigma_{2}\right)$ of the central interaction (6) will only survive in the folded potential for those colliding systems in which each nucleus has a non-zero angular momentum. (Hence, for example, these parts could not be invoked to explain the anomaly for the scattering of ${ }^{6} \mathrm{Li}$ from spin-zero nuclei even though ${ }^{6} \mathrm{Li}$ has spin-one.) The spin angular momentum transfer is uniquely $S=1$ for these interaction terms $[46,146]$. Then parity conservation precludes a total angular momentum transfer of 0 to either nucleus when the spin-spin interaction acts in first order $[46,146]$. Consequently, the contribution of these terms to the cross section is incoherent with the predominant ones from the monopole Coulomb and nuclear interactions; there is no interference to this order [46, 146].

Further, only a few unpaired nucleons in each nucleus contribute to the $S=1$ potential. However, since the part of the interaction (6) with the longest range, namely the OPEP, has an $S=T=1$ nature, we make a rough estimate of its importance. In fig. 2 we showed that when an OPEP-like interaction is allowed (incorrectly) to act between all pairs of nucleons in the ${ }^{16} \mathrm{O}+{ }^{60} \mathrm{Ni}$, it contributes a potential of about $3 \mathrm{MeV}$ at the strong absorption radius. This potential has an $S=T=1$ character and should not be present for this sytem. However, such a term is allowed for a system such as ${ }^{17} \mathrm{O}+{ }^{59} \mathrm{Co}$. If only one odd nucleon from each nucleus contributed, the strength of this term would be reduced by a factor $\sim\left(A_{1} A_{2}\right)$ to $\sim 0.003 \mathrm{MeV}$. This estimate is probably a lower limit since the odd nucleons participating are the most weakly bound ones and 
typically have mean square radii larger than the nucleus as a whole. However, even if our estimate were too small by an order of magnitude, the $S=1$ potential would still be only $\sim 0.03 \mathrm{MeV}$ which is less than $3 \%$ of the $S=0$ potential.

In conclusion, it should be quite adequate to ignore the $S=1$ and $T=1$ contributions to folded potentials which are used to describe cross sections for elastic scattering or the excitation of states having natural parity. However, one must treat the $S=1$ and $T=1$ terms more carefully when asymmetry, spin transfer, or charge-exchange data are being described. Indeed, when asymmetry data are considered, it is important to include also the nucleon-nucleon spin-orbit interaction $[93,158]$ and perhaps treat the exchange terms [158] more carefully as well.

\section{Appendix B: Momentum-space representation}

In this appendix we give the basic formulae used to evaluate double folding-model potentials and transition potentials for elastic and inelastic scattering respectively. These techniques were incorporated in the computer code DOLFIN, originally written by L.D. Rickertsen. The role of moments in folding calculations is also emphasized.

\section{B.1. Folding formulae in momentum space}

The basic double folding-model formula for local nucleon-nucleon interactions is given by eq. (5). To evaluate this integral, we use a $k$-space method similar to those outlined in $[33,159]$ (see also [49] where an analytic development is given using Woods-Saxon forms for the densities). If we denote the Fourier transform of a function $f(r)$ by

$$
\tilde{f}(\boldsymbol{k})=\int \mathrm{d} \boldsymbol{r} \exp (\mathrm{i} \boldsymbol{k} \cdot \boldsymbol{r}) f(\boldsymbol{r})
$$

then $v\left(r_{12}\right)$ in eq. (5) becomes (see fig. 1)

$$
v\left(\boldsymbol{r}_{12}\right)=(2 \pi)^{-3} \int \mathrm{d} \boldsymbol{k} \tilde{v}(\boldsymbol{k}) \exp \left[-\mathrm{i} \boldsymbol{k} \cdot\left(\boldsymbol{R}+\boldsymbol{r}_{2}-\boldsymbol{r}_{1}\right)\right]
$$

and

$$
U_{\mathrm{F}}(\boldsymbol{R})=(2 \pi)^{-3} \int \mathrm{d} \boldsymbol{k} \exp (-\mathrm{i} \boldsymbol{k} \cdot \boldsymbol{R}) \tilde{v}(\boldsymbol{k}) \tilde{\rho}_{1}(\boldsymbol{k}) \tilde{\rho}_{2}(-\boldsymbol{k}),
$$

with

$$
\tilde{U}_{\mathrm{F}}(\boldsymbol{k})=\int \mathrm{d} \boldsymbol{R} \exp (\mathrm{i} \boldsymbol{k} \cdot \boldsymbol{R}) U_{\mathrm{F}}(\boldsymbol{R})=\tilde{v}(\boldsymbol{k}) \tilde{\rho}_{1}(\boldsymbol{k}) \tilde{\rho}_{2}(-\boldsymbol{k}) .
$$

It is convenient to display the rotational properties of the above densities. The spin-scalar, isoscalar density is defined by the matrix element

$$
\rho(\boldsymbol{r})=\left\langle\mathrm{A}^{\prime}\left|\sum_{i} \delta\left(\boldsymbol{r}-\boldsymbol{r}_{i}\right)\right| \mathrm{A}\right\rangle
$$

where the sum runs over all nucleons. $A^{\prime}=A$ yields the ground-state density distribution, while 
$\mathrm{A}^{\prime} \neq \mathrm{A}$ gives the transition density for $\mathrm{A} \rightarrow \mathrm{A}^{\prime}$. With the multipole expansion of the delta function, we find

$$
\rho(\boldsymbol{r})=\sum_{L}\left\langle I_{\mathrm{A}^{\prime}} M_{\mathrm{A}^{\prime}} \mid I_{\mathrm{A}} L M_{\mathrm{A}} m\right\rangle Y_{L m}^{*}(\hat{\boldsymbol{r}})\left\langle\mathrm{A}^{\prime}\left\|\sum_{i} r_{i}^{-2} \delta\left(r-r_{i}\right) Y_{L}\left(\hat{\boldsymbol{r}}_{i}\right)\right\| \mathrm{A}\right\rangle,
$$

where the reduced matrix element is as defined by Brink and Satchler [160]. We define

$$
C_{L} \rho_{L}(r)=\left\langle\mathrm{A}^{\prime}\left\|\sum_{i} r_{i}^{-2} \delta\left(r-r_{i}\right) Y_{L}\left(\hat{r}_{i}\right)\right\| \mathrm{A}\right\rangle
$$

Here the normalization constant $C_{L}$ is to be chosen later for convenience. Using (B.1) for a particular $L$ from the sum (B.6), the transform $\tilde{\rho}$ appearing in eq. (B.3) becomes

$$
\tilde{\rho}(\boldsymbol{k})=\left\langle I_{\mathrm{A}^{\prime}}, M_{\mathrm{A}^{\prime}} \mid I_{\mathrm{A}} L M_{\mathrm{A}} m\right\rangle \mathrm{i}^{L} C_{L} \tilde{\rho}_{L}(k) Y_{L m}^{*}(\hat{\boldsymbol{k}})
$$

with

$$
\tilde{\rho}_{L}(k)=4 \pi \int_{0}^{\infty} r^{2} \mathrm{~d} r j_{L}(k r) \rho_{L}(r)
$$

With this result, the folded potential (B.3) can be expressed as

$$
\begin{aligned}
U_{\mathrm{F}}\left(\boldsymbol{R} ; \mathrm{A}_{1} \rightarrow \mathrm{A}_{1}^{\prime}, \mathrm{A}_{2} \rightarrow \mathrm{A}_{2}^{\prime}\right)= & \sum_{L L_{1}^{\prime} L_{2}}\left\langle L M \mid L_{1} L_{2} m_{1} m_{2}\right\rangle\left\langle I_{1}^{\prime} M_{1}^{\prime} \mid I_{1} L_{1} M_{1} m_{1}\right\rangle \\
& \times\left\langle I_{2}^{\prime} M_{2}^{\prime} \mid I_{2} L_{2} M_{2} m_{2}\right\rangle C_{L} U_{L}(R) Y_{L M}^{*}(\hat{R}) .
\end{aligned}
$$

Here we have used subscript 1 for quantities belonging to nucleus $A_{1}$ and 2 for quantities belonging to nucleus $A_{2}$. The potential (B.10) contains contributions in which both the multipoles $L_{1}$ and $L_{2}$ may be non-zero. It includes as special cases mutual excitation (both nuclei excited, $A_{1}^{\prime} \neq A_{1}$ and $A_{2}^{\prime} \neq A_{2}$ ), single excitation (one nucleus excited, $A_{1}^{\prime} \neq A_{1}$ or $A_{2}^{\prime} \neq A_{2}$ ) and elastic scattering $\left(A_{1}^{\prime}=A_{1}, A_{2}^{\prime}=A_{2}\right)$. Also in eq. (B.10) we have

$$
C_{L} U_{L}(R)=\frac{C_{L_{1}} C_{L_{2}}}{(4 \pi)^{1 / 2}} \mathrm{i}^{L_{1}-L_{2}-L} \frac{\hat{L}_{1} \hat{L}_{2}}{\hat{L}}\left\langle L 0 \mid L_{1} L_{2} 00\right\rangle \frac{1}{2 \pi^{2}} \int_{0}^{\infty} k^{2} \mathrm{~d} k j_{L}(k R) \tilde{v}(k) \tilde{\rho}_{L_{1}}(k) \tilde{\rho}_{L_{2}}(k) .
$$

Here $v$ has been assumed to be a scalar in coordinate space and $\hat{x}=(2 x+1)^{1 / 2}$. It is convenient to choose the $C_{L}$ factors to be

$$
C_{L}= \begin{cases}(4 \pi)^{1 / 2} & \text { if } L=0 \\ 0 & \text { if } L \neq 0\end{cases}
$$

With this convention $U_{0}(R)$ is the spherical part of the optical potential and $\rho_{L}(r)$ with $L=0$ is the ordinary density for a nucleus of spin zero. In the most common case of single excitation of a spin-zero nucleus induced by an ion with zero spin, eq. (B.11) becomes especially simple. For this we have $I_{2}^{\prime}=I_{2}=L_{2}=0$, say, and $I_{1}=0, I_{1}^{\prime}=L_{1}=L$. Then

$$
U_{\mathbf{F}}(\boldsymbol{R})=C_{L} U_{L}(R) Y_{L M}^{*}(\hat{\boldsymbol{R}})
$$


and

$$
U_{L}(R)=\frac{1}{2 \pi^{2}} \int k^{2} \mathrm{~d} k j_{L}(k R) \tilde{v}(k) \tilde{\rho}_{L}(k) \tilde{\rho}_{0}(k) .
$$

The potentials for elastic scattering discussed in this paper correspond to $L=0$ in this equation.

If we make the associations: $s_{\mathrm{a}}=I_{1}, s_{\mathrm{b}}=I_{1}^{\prime}, J_{\mathrm{A}}=I_{2}, J_{\mathbf{B}}=I_{2}^{\prime}, L=L, S=L_{1}$ and $J=L_{2}$, we may identify the form factor $G_{L S J}(r)$ defined in [146] and often used in DWBA calculations. In particular,

$$
G_{L L_{1} L_{2}}(R)=\mathrm{i}^{L} \frac{\hat{L}_{1}^{\prime}}{\hat{L}_{1} \hat{L}_{2}}(-)^{I_{1}^{\prime}-I_{1}-L_{1}} C_{L} U_{L}(R) .
$$

The discussion above has ignored spin and isospin; that is, it is appropriate for the $S=T=0$ component $v_{00}$. The extension to $S=1$ and/or $T=1$, analogous to the discussion in appendix $\mathrm{A}$, is obvious. For example, the operator for electric transitions is

$$
Q_{L M}=e \sum_{\mathrm{p}} r_{\mathrm{p}}^{L} Y_{L M}\left(\hat{\boldsymbol{r}}_{\mathrm{p}}\right)
$$

summed over protons only. We consider this case because we used measured $\mathrm{E} L$ transition rates to normalize our transition densities. The conventional reduced transition probability [148] is defined as

$$
B\left(\mathrm{E} L ; \mathrm{A} \rightarrow \mathrm{A}^{\prime}\right)=\frac{2 I_{\mathrm{A}^{\prime}}+1}{2 I}\left|\left\langle\mathrm{~A}^{\prime} \| Q_{L}+\mid \mathrm{A}\right\rangle\right|^{2} .
$$

Applying the analogue of eqs. (B.6) and (B.7), this can soon be written

$$
B\left(\mathrm{E} L ; \mathrm{A} \rightarrow \mathrm{A}^{\prime}\right)=\frac{2 I_{\mathrm{A}^{\prime}}+1}{2 I_{\mathrm{A}}+1} e^{2}\left|\int \mathrm{d} r r^{L+2} \rho_{\mathrm{p}, L}(r)\right|^{2}
$$

where we assume $L \neq 0$ and $\rho_{\mathrm{p}, L}$ is the proton part of the transition density,

$$
\rho_{L}(r)=\rho_{\mathrm{p}, L}(r)+\rho_{\mathrm{n}, L}(r) .
$$

\section{B.2. Role of moments in $k$-space folding}

In addition to being computationally fast and convenient, the $k$-space formulae also provide a number of useful relations [151] involving the moments of the densities and potentials. In particular, the small- $k$ limit of (B.4) gives

$$
J_{0}(U)=J_{0}(v) J_{0}\left(\rho_{1}\right) J_{0}\left(\rho_{2}\right)
$$

where $J_{0}(f)$ is the volume integral of the function $f$. This result is only useful when $\rho_{1}, \rho_{2}$ and $v$ are all scalars. When $\rho_{1}$ and $v$ are scalars while $\rho_{2} \propto Y_{L M}^{*}$ (as is the case for single excitation), the small-k limit gives

$$
J_{L}\left(U_{L}\right)=J_{0}(v) \dot{J_{0}}\left(\rho_{1}\right) J_{L}\left(\rho_{2}\right)
$$


where

$$
J_{L}\left(f_{L}\right)=4 \pi \int_{0}^{\infty} \mathrm{d} r r^{L+2} f_{L}(r)
$$

and $\rho_{L}$ and $U_{L}$ are defined in eqs. (B.7) and (B.11). These equalities and their generalizations [151] provide useful numerical checks on the calculated folded potentials.

The $k$-space folding method is also convenient when charge distributions inferred from electron scattering are to be used for folding. In particular, the $\rho_{i}(r)$ appearing in eq. (5) are distributions of the centers of mass of the nucleons (often called "point-densities") whereas one obtains charge densities (and transition densities) from electron scattering. These are related in $k$-space by

$$
\tilde{\rho}_{\mathrm{ch}}(\boldsymbol{k})=\tilde{\rho}_{\mathrm{N}}(\boldsymbol{k}) \tilde{\rho}_{\mathrm{n}}(k)+\tilde{\rho}_{\mathrm{P}}(\boldsymbol{k}) \tilde{\rho}_{\mathrm{p}}(k)
$$

where $\mathrm{n}$ and $\mathrm{p}$ denote nuclear point densities to be used in (B.3), $\tilde{\rho}_{\mathrm{ch}}(\boldsymbol{k})$ is the Fourier transform of the nuclear charge density and $\tilde{\rho}_{\mathrm{N}}$ and $\tilde{\rho}_{\mathrm{P}}$ are the Fourier transforms of the charge distributions of the neutron and proton, respectively. If it is assumed that $\rho_{\mathrm{n}} \simeq(N / Z) \rho_{\mathrm{p}}$ as is often done for nuclei with $N \approx Z$, the proton and neutron point densities appearing in eq. (B.11) are readily found from

$$
\tilde{\rho}_{\mathrm{p}}=\frac{Z}{N} \tilde{\rho}_{\mathrm{n}}=\tilde{\rho}_{\mathrm{ch}} /\left(\tilde{\rho}_{\mathrm{P}}+\frac{N}{Z} \tilde{\rho}_{\mathrm{N}}\right)
$$

so that unfolding the finite-sized charge distributions of the nucleons is a simple operation of division in $k$-space. We have used an exponential form for $\rho=\rho_{\mathrm{P}}+(N / Z) \rho_{\mathrm{N}}$,

$$
\rho(r)=\frac{\alpha^{3}}{8 \pi} \mathrm{e}^{-\alpha r} ; \quad \tilde{\rho}(k)=\frac{\alpha^{4}}{\left(\alpha^{2}+k^{2}\right)^{2}}
$$

which has $\left\langle r^{2}\right\rangle=12 / \alpha^{2}$. Then $\alpha$ was chosen so that (see section 3.4.1)

$$
\left\langle r^{2}\right\rangle=0.76-0.11(N / Z) \mathrm{fm}^{2} \text {. }
$$

The $k$-space formulae also may be used to estimate changes in the folded potential due to small variations in the densities and in the nucleon-nucleon interaction. To the extent that these variations are small and predominantly characterized by changes in the mean square radii, we may use the relation obtained by expanding to order $k^{2}$. For scalar functions $\left(L=L_{1}=L_{2}=0\right)$ this is

$$
\tilde{U}(k)=\tilde{U}_{0}(k)+\delta \tilde{U}(k) \approx \tilde{v}(k) \tilde{\rho}_{1}(k) \tilde{\rho}_{2}(k)\left[1-\frac{1}{6} k^{2} \sum \delta\left\langle r^{2}\right\rangle\right]
$$

where $\rho_{1}, \rho_{2}$ and $v$ are to be regarded as reference distributions and $\sum \delta\left\langle r^{2}\right\rangle$ denotes the sum of the changes in their mean-square radii. The corresponding change in $U(R)$ is then characterized by

$$
\frac{\partial U(R)}{\partial\left\langle r^{2}\right\rangle}=-\frac{1}{12 \pi^{2}} \int_{0}^{\infty} k^{4} \mathrm{~d} k j_{0}(k R) \tilde{v}(k) \tilde{\rho}_{1}(k) \tilde{\rho}_{2}(k) .
$$

This gives

$$
\frac{\partial U(R)}{\partial\left\langle r^{2}\right\rangle}=\frac{1}{6} \frac{\partial^{2} U(R)}{\partial R^{2}}+\frac{1}{3 R} \frac{\partial U(R)}{\partial R} .
$$


Near the important strong absorption radius, $U$ may be roughly characterized as a decaying exponential $\sim \mathrm{e}^{-\mathrm{R} / \alpha}$ giving

$$
\frac{\partial U(R)}{\partial\left\langle r^{2}\right\rangle} \approx \frac{U(R)}{6 \alpha^{2}}\left[1-\frac{2 \alpha}{R}\right] \approx \frac{U(R)}{6 \alpha^{2}} .
$$

A typical value of $\alpha$ is $0.65 \mathrm{fm}$ (see table 3 ); in this case we would have $\delta U / U \approx 0.4 \delta\left\langle r^{2}\right\rangle$ if $\left\langle r^{2}\right\rangle$ were expressed in $\mathrm{fm}^{2}$. Thus, once $U(R)$ is calculated near the strong absorption radius, changes in $U$ due to using slightly different densities may be estimated readily by using eq. (B.26). For example, the overall behavior of $\partial U / \partial\left\langle r^{2}\right\rangle$ exhibited by the dashed line in fig. 5 is easily estimated in this way. Figure 3 can be qualitatively understood in the same way. Clearly, those changes in shape which leave $\left\langle r^{2}\right\rangle$ unchanged require an extension of this method to higher moments. It is felt that small changes in $\left\langle r^{2}\right\rangle$ occur frequently enough to make the above estimate useful.

A moment expansion like that indicated in eq. (9) is especially simple in $k$-space if one makes the replacement

$$
\tilde{v}(k) \rightarrow J_{0}\left[1-\frac{1}{6}\left\langle r^{2}\right\rangle k^{2}+\frac{1}{120}\left\langle r^{4}\right\rangle k^{4}+\ldots\right]
$$

in (B.11), retaining enough moments to achieve the desired accuracy. Although this expansion is not especially convenient for the interaction in eq. (10), it could be useful for interactions of shorter range or for exploring the sensitivity of folding potentials to higher moments.

\section{Appendix C: Density-dependent interactions}

Recently, a number $[2,5,6,50,86]$ of realistic density-dependent interactions derived from $G$-matrices have been used in scattering calculations for both light and heavy ions. In a localdensity approximation the use of such interactions in double-folding calculations typically amounts to replacing $v\left(r_{12}\right)$ in eq. (5) by $v\left(r_{12}, \rho\right)$ where $\rho$ is the density midway between the two interacting nucleons,

$$
\rho=\rho_{1}\left(\left|\boldsymbol{r}_{1}+\frac{1}{2} \boldsymbol{r}_{12}\right|\right)+\rho_{2}\left(\left|\boldsymbol{r}_{2}-\frac{1}{2} \boldsymbol{r}_{12}\right|\right)
$$

and $\rho_{i}$ denotes the ground-state density of the ith nucleus. Often $v\left(r_{12}, \rho\right)$ contains a term proportional to some fractional power of $\rho$ and this makes evaluation of the folding integral of eq. (5) much more difficult. We have found [39] that the density dependence of $v\left(r_{12}, \rho\right)$ can, for realistic interactions, be represented well by the exponential form

$$
v\left(r_{12}, \rho\right)=v_{1}\left(r_{12}\right)+v_{2}\left(r_{12}\right) \mathrm{e}^{-\beta \rho} \text {. }
$$

Since the range of the scalar-isoscalar part of realistic interactions is quite short, there should be little change if we use

$$
\rho=\rho_{1}\left(r_{1}\right)+\rho_{2}\left(r_{2}\right)
$$

instead of the prescription (C.1). (If the results of using these two prescriptions are significantly different, then doubt is cast on the concept of using a local density approximation.) When this is done, eq. (C.2) becomes

$$
v\left(r_{12}, \rho\right)=v_{1}\left(r_{12}\right)+v_{2}\left(r_{12}\right) \mathrm{e}^{-\beta \rho_{1}\left(r_{1}\right)} \mathrm{e}^{-\beta \rho_{2}\left(r_{2}\right)}
$$


The factorization of the second term allows the $k$-space method to retain its simple form. Technically, this is done by replacing $\rho_{i}\left(r_{i}\right)$ by $\rho_{i}\left(r_{i}\right) \exp \left[-\beta \rho_{i}\left(r_{i}\right)\right]$ in eq. (5) (or the corresponding replacement of their Fourier transforms in eq. (B.3)) when evaluating the contribution from the second term on the right of eq. (C.4).

The DDD interaction discussed in section 3.3 .3 with which we made some exploratory calculations was treated in this way. The oscillator matrix elements of $\bar{G}(r, \rho)$ that we were given [50] could be represented well by the form (C.2) with $\beta=41.4 \mathrm{fm}^{3}$ and using a sum of Yukawa terms for $v_{1}$ and $v_{2}$ analogous to those used for the M3Y interaction. For example, the $S=T=0$ part of this interaction has

$$
\begin{aligned}
& v_{1}(r)=\left[6839 \frac{\mathrm{e}^{-4 r}}{4 r}-1887 \frac{\mathrm{e}^{-2.5 r}}{2.5 r}\right] \mathrm{MeV}, \\
& v_{2}(r)=\left[6893 \frac{\mathrm{e}^{-4 r}}{4 r}-1938 \frac{\mathrm{e}^{-2.5 r}}{2.5 r}\right] \mathrm{MeV},
\end{aligned}
$$

which may be compared with the M3Y interactions (10) and (11). The volume integral of $v_{1}$ is $J_{1}=-174.4 \mathrm{MeV} \mathrm{fm}^{3}$, that of $v_{2}$ is $J_{1}=-205.0 \mathrm{MeV} \mathrm{fm}^{3}$. At zero density these result in $J_{00}(\rho=0)=-379.4 \mathrm{MeV} \mathrm{fm}^{3}$, while at "normal" density, $\rho=\rho_{0} \approx 0.17 \mathrm{fm}^{-3}$, the $v_{2}$ term contributes almost nothing so that $J_{00}\left(\rho=\rho_{0}\right)=-174.6 \mathrm{MeV} \mathrm{fm}^{3}$. Single-nucleon exchange was included by using the (density-independent) zero-range pseudo-potential of eq. (15) normalized by comparison with some "exact" calculations of proton scattering just as with the M3Y interaction. This resulted in $\hat{J}_{00}=-213 \mathrm{MeV} \mathrm{fm}^{3}$. At a density which is one-third of "normal", $\rho=\frac{1}{3} \rho_{0}$, the DDD interaction then has $J_{00}=-194 \mathrm{MeV} \mathrm{fm}^{3}$, or with exchange included, $J_{00}^{\prime}=-407 \mathrm{MeV} \mathrm{fm}{ }^{3}$ and a mean square radius $\left\langle r^{2}\right\rangle=2.58 \mathrm{fm}^{2}$. These values are almost exactly the same as for the density-independent M3Y interaction.

Very recently [86], calculations have been made with an interaction which include a densitydependent factor $\exp (-\alpha \rho)$ in the $v_{1}$ term as well. A form [57] very similar to eq. (C.2) has also been used to estimate the effects of single-nucleon exchange in the Slater approximation; it can be handled in the same way.

\section{References}

[1] W.E. Frahn and K.E. Rehm, Phys. Reports 37C (1978) 1.

[2] B. Sinha, Phys. Reports 20C (1975) 1.

[3] R.C. Barrett and D.F. Jackson, Nuclear Sizes and Structure (Clarendon Press, Oxford, 1977).

[4] G.W. Greenlees, G.J. Pyle and Y.C. Tang, Phys. Rev. 171 (1968) 1115.

[5] J.P. Jeukenne, A. Lejeune and C. Mahaux, Phys. Rev. C16 (1977) 80.

[6] F.A. Brieva and J.R. Rook, Nucl. Phys. A291 (1977) 317;

F.A. Brieva, H.V. Geramb and J.R. Rook, Phys. Lett. 79B (1978) 177.

[7] C.B. Dover and J.P. Vary, Phys. Rev. Lett. 31 (1973) 1510.

[8] G.R. Satchler, in: Proc. Int. Conf. on Reactions between Complex Nuclei, Nashville, Tenn., 1974 (North-Holland Publishing Co., Amsterdam, 1974).

[9] B. Sinha, Phys. Rev. Lett. 33 (1974) 600; Phys. Rev. C11 (1975) 1546.

[10] B. Buck, C.B. Dover and J.P. Vary, Phys. Rev. C11 (1975) 1803.

[11] G. Bertsch, J. Borysowicz, H. McManus and W.G. Love, Nucl. Phys. A284 (1977) 399.

[12] J.P. Elliott, A.D. Jackson, H.A. Mavromatis, E.A. Sanderson and B. Singh, Nucl. Phys, A121 (1968) 241.

[13] D.M. Brink, Eur. Conf. Nucl. Phys. Heavy Ions, Caen, 1976, J. de Phys. C-5 (1976) 47.

[14] W.U. Schröder and J.R. Huizenga, Ann. Rev. Nucl. Sci. 27 (1977) 465. 
[15] Proc. Symp. Heavy Ion Elastic Scattering, ed. R.M. De Vries, University of Rochester (1977).

[16] R. Bass, Phys. Rev. Lett. 39 (1977) 265.

[17] J.R. Birkelund and J.R. Huizenga, Phys. Rev. C17 (1978) 126.

[18] H.J. Krappe, J.R. Nix and A.J. Sierk, Phys. Rev. Lett. 42 (1979) 215, and to be published.

[19] P.R. Christensen and A. Winther, Phys. Lett. 65B (1977) 19.

[20] H. Feshbach, Ann. Phys. (N.Y.) 19 (1967) 287.

[21] J. Fleckner and U. Mosel, Nucl. Phys. A277 (1977) 170.

[22] U. Mosel, Particles and Nuclei 3 (1972) 297;

N. Austern, in: Heavy-Ion Scattering, Argonne National Laboratory Report ANL-7837 (1971) p. 293.

[23] N. Vinh Mau, Phys. Lett. 71B (1977) 5.

[24] W.G. Love, T. Terasawa and G.R. Satchler, Nucl. Phys. A291 (1977) 183.

[25] B. Sinha, Phys. Rev. Lett. 42 (1979) 690.

[26] D.M. Brink and N. Takigawa, Nucl. Phys. A279 (1977) 159.

[27] N. Rowley, H. Doubre and C. Marty, Phys. Lett. 69B (1977) 147.

[28] A.J. Baltz and S. Kahana, Phys. Rev. C17 (1978) 555.

[29] C.B. Fulmer et al., to be published.

[30] K.S. Toth, J.L.C. Ford, G.R. Satchler, E.E. Gross, D.C. Hensley, S.T. Thornton and T.C. Schweizer, Phys. Rev. C14 (1976) 1471.

[31] H. Amakawa and K.I. Kubo, Nucl. Phys. A266 (1976) 521;

W. Weiss, P. Egelhof, K.D. Hildebrand, D. Kassen, M. Makowska-Rzeszutko, D. Fick, H. Ebinghaus, E. Steffens, A. Amakawa and K.I. Kubo, Phys. Lett. 61B (1976) 237;

F. Petrovich, D. Stanley, L.A. Parks and P. Nagel, Phys. Rev. C17 (1978) 1642.

[32] L.T. Chua, F.D. Becchetti, J. Janecke and F.L. Milder, Nucl. Phys. A273 (1976) 243.

[33] P.J. Moffa, C.B. Dover and J.P. Vary, Phys. Rev. C16 (1977) 1857.

[34] G.R. Satchler and C.B. Fulmer, Phys. Lett. 50B (1974) 309.

[35] G.R. Satchler, M.L. Halbert, N.M. Clarke, E.E. Gross, C.B. Fulmer, A. Scott, D. Martin, M.D. Cohler, D.C. Hensley, C.A. Ludemann, J.G. Cramer, M.S. Zisman and R.M. DeVries, Nucl. Phys. A298 (1978) 313.

[36] R.A. Broglia and A. Winther, Phys. Reports 4C (1972) 153;

D.M. Brink and N. Rowley, Nucl. Phys. A219 (1974) 79;

D.H.E. Gross and H. Kalinowski, Phys. Lett. 48B (1974) 302.

[37] G.R. Satchler, Phys. Lett. 59B (1975) 121.

[38] L.D. Rickertsen and G.R. Satchler, Phys. Lett. 66B (1977) 9.

[39] W.G. Love, Phys. Lett. 72B (1977) 4;

F. Petrovich, D. Stanley and J.J. Bevelacqua, Phys. Lett. 71B (1977) 259.

[40] P.W. Coulter and G.R. Satchler, Nucl. Phys. A293 (1978) 269.

[41] I. Reichstein and Y.C. Tang, Nucl. Phys. A139 (1969) 144.

[42] E.C. Halbert and G.R. Satchler, Nucl. Phys. A233 (1974) 265; G.R. Satchler, Z. Physik 260 (1973) 209.

[43] C.B. Dover and J.P. Vary, in: Proc. Symp. on Classical and Quantum Mechanical Aspects of Heavy-Ion Collisions, Heidelberg, Germany, 1974 (Springer-Verlag, Berlin, 1975).

[44] C.B. Dover, P.J. Moffa and J.P. Vary, Phys. Lett. 56B (1975) 4.

[45] P.J. Moffa, J.P. Vary, C.B. Dover, C.W. Towsley, R.G. Hanus and K. Nagatani, Phys. Rev. Lett. 35 (1975) 992

[46] W.G. Love and G.R. Satchler, Nucl. Phys. A159 (1970) 1.

[47] J.B. Ball, C.B. Fulmer, E.E. Gross, M.L. Halbert, D.C. Hensley, C.A. Ludemann, M.J. Saltmarsh and G.R. Satchler, Nucl. Phys. A252 (1975) 208.

[48] G.R. Satchler and W.G. Love, Phys. Lett. 65B (1976) 415.

[49] L.J.B. Goldfarb, Nucl. Phys. A301 (1978) 497.

[50] Y. Eisen and B. Day, Phys. Lett. 63B (1976) 253; B. Day, private communication.

[51] J.Y. Park, W. Scheid and W. Greiner, Phys. Rev. C6 (1972) 1565.

[52] P.G. Zint and U. Mosel, Phys. Rev. C14 (1976) 1488.

[53] G.H. Goritz and U. Mosel, Z. Physik A277 (1976) 243; P.G. Zint, Z. Physik A281 (1977) 373.

[54] M. LeMere and Y.C. Tang, Phys. Rev. C19 (1979) 391.

[55] W.G. Love and L.W. Owen, Nucl. Phys. A239 (1975) 74.

[56] M. Golın, F. Petrovich and D. Robson, Phys. Lett. 64B (1976) 253.

[57] W.G. Love, Nucl. Phys. A312 (1978) 160.

[58] F. Borkowski, G.G. Simon, V.H. Waltner and R.D. Wendling, Z. Physik A275 (1975) 29. 
[59] C.W. de Jager, H. de Vries and C. de Vries, Atomic Data and Nuclear Data Tables 14 (1974) 479.

[60] J.W. Negele, Phys. Rev. C1 (1970) 1260; C9 (1974) 1054.

[61] Y. Eisen, Phys. Lett. 37B (1971) 33.

[62] C.F. Clement, Nucl. Phys. A213 (1973) 469; C.M. Shakin and M.S. Weiss, Phys. Rev. C15 (1977) 1911.

[63] F. Malaguti, A. Uguzzoni, E. Verondini and P.E. Hodgson, Nucl. Phys. A297 (1978) 287.

[64] C.J. Batty and G.W. Greenlees, Nucl. Phys. A133 (1969) 673.

[65] A. Chaumeaux, V. Layly and R. Schaeffer, Ann. Phys. (N.Y.) 116 (1978) 247.

[66] G.C. Li, M.R. Yearian and I. Sick, Phys. Rev. C9 (1974) 1861.

[67] A. Budzanowski, A. Dudek, K. Grotowski, Z. Majka and A. Strzalkowski, Particles and Nuclei 6 (1973) 97.

[68] W.G. Love, Phys. Rev. C17 (1978) 1876.

[69] D.A. Goldberg, Phys. Lett. 55B (1975) 59;

D.A. Goldberg, S.M. Smith and G.F. Burdzik, Phys. Rev. C10 (1974) 1362;

A. Budzanowski, H. Dabrowski, L. Freindl, K. Grotowski, S. Micek, R. Planeta, A. Strzalkowski, M. Bosman, P. Leleux, P. Macq, J.P. Meulders and C. Pirat, Phys. Rev. C17 (1978) 951.

[70] F. Michel and R. Vanderpoorten, Phys. Rev. C16 (1977) 142;

H.P. Gubler, U. Kiebele, H.O. Meyer, G.R. Plattner and I. Sick, Phys. Lett. 74B (1978) 202;

Th. Delbar, Gh. Gregoire, G. Paic, R. Ceuleneer, F. Michel, R. Vanderpoorten, A. Budzanowski, H. Dabrowski, L. Friendl, K. Grotowski, S. Micek, R. Planeta, A. Strzalkowsk1 and K.A. Eberhard, Phys. Rev. C18 (1978) 1237.

[71] N. Takigawa, S.Y. Lee and C. Marty, Phys. Lett. 76B (1978) 187; S.Y. Lee, N. Takigawa and C. Marty, Nucl. Phys. A308 (1978) 161.

[72] R.M. Wieland, R.G. Stokstad, G.R. Satchler and L.D. Rickertsen, Phys. Rev. Lett. 37 (1976) 1458, and to be published.

[73] G.R. Satchler and W.G. Love, Phys. Lett. 76B (1978) 23.

[74] B. Buck and A.A. Pilt, Nucl. Phys. A280 (1977) 133.

[75] J. Blocki, J. Randrup, W.J. Swiatecki and C.F. Tsang, Ann. Phys. 105 (1977) 427.

[76] D.M. Brink and Fl. Stancu, Nucl. Phys. A299 (1978) 321.

[77] Z. Majka, A. Budzanowski, K. Grotowski and A. Strzalkowski, Phys. Rev. C18 (1978) 114.

[78] F.D. Becchetti and G.W. Greenlees, Phys. Rev. 182 (1969) 1190.

[79] G.W. Greenlees, G.J. Pyle and Y.C. Tang, Phys. Rev. 171 (1968) 1115;

G.W. Greenlees, W. Makofske and G.J. Pyle, Phys. Rev. C1 (1970) 1145.

[80] W.G. Love, Phys. Rev. C15 (1977) 1261.

[81] E. Friedman, Phys. Lett. 29B (1969) 213; W.D. Myers, Nucl. Phys. A294 (1973) 465.

[82] F. Petrovich, Proc. Workshop on Microscopic Optical Potentials, Hamburg, 1978, ed. H.V. Geramb (Lecture notes in Physics 89 , Springer-Verlag, Berlın, 1979).

[83] D.E. Bainum, R.W. Finlay, J. Rapaport, J.D. Carlson and W.G. Love, Phys. Rev. C16 (1977) 1377.

[84] B. Buck, H. Friedrich and C. Wheatley, Nucl. Phys. A275 (1977) 246;

H. Friedrich and L.F. Canto, Nucl. Phys. A291 (1977) 249.

[85] J.S. McCarthy, I. Sick and R.R. Whitney, Phys. Rev. C15 (1977) 1396.

[86] Z. Majka, H.J. Gils and H. Rebel, Z. Physik A288 (1978) 139.

[87] F.G. Perey, modified by L.W. Owen (unpublished).

[88] D.L. Hillis, E.E. Gross, D.C. Hensley, C.R. Bingham, F.T. Baker and A. Scott, Phys. Rev. C16 (1977) 1467.

[89] A. Dudek-Ellss, V. Shkolnik and D. Dehnhard, Phys. Rev. C18 (1978) 1039.

[90] H. Wojciechowski, N.B.J. Tannous, R.H. Davis, D. Staneley, M. Golin and F. Petrovich, Phys. Rev. C17 (1978) 2126.

[91] N.B.J. Tannous, J.F. Mateja, D.C. Wilson, L.R. Medsker and R.H. Davis, Phys. Rev. C18 (1978) 2190.

[92] L.A. Parks, K.W. Kemper, A.H. Lumpkin, R.I. Cutler, L.H. Harwood, D. Stanley, P. Nagel and F. Petrovich, Phys. Lett. 70B (1977) 27.

[93] F. Petrovich, D. Stanley, L.A. Parks and P. Nagel, Phys. Rev. C17 (1978) 1642.

[94] G.R. Satchler, Nucl. Phys. A279 (1977) 493.

[95] G.R. Satchler, Phys. Lett. 55B (1975) 167.

[96] D. Vinciguerra and T. Stovall, Nucl. Phys. A132 (1969) 410.

[97] D.F. Jackson and R.C. Johnson, Phys. Lett. 49B (1974) 249.

[98] D.G. Kovar, private communication (1978).

[99] K.E. Rehm, W. Henning, J.R. Erskine and D.G. Kovar, Phys. Rev. Lett. 40 (1978) 1479.

[100] S. Kubono, P.D. Bond, C.E. Thorn and M.J. Levine, Bull. Am. Phys. Soc. 23 (1978) 615; S. Kubono, P.D. Bond, C.E. Thorn and M.J. Levine, Phys. Lett. 81 B (1979) 140.

[101] K.O. Groenveld, L. Meyer-Schutzmeister, A. Richter and U. Strohbusch, Phys. Rev. C6 (1972) 805.

[102] F. Videbaek, R.B. Goldstein, L. Grodzins, S.G. Steadman, T.A. Belote and J.D. Garrett, Phys. Rev. C15 (1977) 954. 
[103] S.C. Pieper, M.H. Macfarlane, D.H. Gloeckner, D.G. Kovar, F.D. Becchetti, B.G. Harvey, D.L. Hendrie, H. Homeyer, J. Mahoney, F. Puhlhofer, W. von Oertzen and M.S. Zisman, Phys. Rev. C18 (1978) 180;

C. Olmer, M. Mermaz, M. Buenerd, C.K. Gelbke, D.L. Hendrie, J. Mahoney, D.K. Scott, M.H. Macfarlane and S.C. Pieper, Phys. Rev. C18 (1978) 205.

[104] V. Shkolnik, D. Dehnard, S. Kubono, M.A. Franey and S. Tripp, Phys. Lett. 74B (1978) 195.

[105] P. Braun-Munzinger, G.M. Berkowitz, T.M. Cormier, C.M. Jachcinski, J.W. Harris, J. Barrette and M.J. LeVine, Phys. Rev. Lett. 38 (1977) 944.

[106] K.O. Terenetski and J.D. Garrett, Phys. Rev. C18 (1978) 1944.

[107] K.G. Nair, H. Voit, C.W. Towsley, M. Hamm, J.D. Bronson and K. Nagatani, Phys. Rev. C12 (1975) 1575.

[108] J.L.C. Ford, K.S. Toth, G.R. Satchler, D.C. Hensley, L.W. Owen, R.M. DeVries, R.M. Gaedke, P.J. Riley and S.T. Thornton, Phys. Rev. C10 (1974) 1429.

[109] R.M. DeVries, D.A. Goldberg, J.G. Cramer, M.L. Halbert and R.G. Stokstad, unpublished.

[110] G.R. Morgan and N.R. Fletcher, unpublished.

[111] S.T. Thornton, D.E. Gustafson, J.L.C. Ford, K.S. Toth and D.C. Hensley, Phys. Rev. C13 (1976) 1502.

[112] N. Anantaraman, Phys. Rev. C8 (1973) 2245.

[113] J.C. Hiebert and G.T. Garvey, Phys. Rev. 135 (1964) B346.

[114] D.S. Gale and J.S. Eck, Phys. Rev. C7 (1973) 1950.

[115] J.G. Cramer, R.M. DeVries, D.A. Goldberg, M.S. Zisman and C.F. Maguire, Phys. Rev. C14 (1976) 2158.

[116] V. Shkolnik, D. Dehnard, S. Kubono, M.A. Franey and S. Tripp, Phys. Lett. 74B (1978) 195.

[117] K.O. Groeneveld, L. Meyer-Schutzmeister, A. Richter and U. Strohbusch, Phys. Rev. C6 (1972) 805.

[118] D.G. Kovar, W. Henning, B. Zeidman, Y. Eisen, J.R. Erskine, H.T. Fortune, T.R. Ophel, P. Sperr and S.E. Vigdor, Phys. Rev. C17 (1978) 83.

[119] K.E. Rehm, H.J. Korner, M. Richter, H.P. Rother, J.P. Schiffer and H. Spieler, Phys. Rev. C12 (1975) 1945.

[120] E. Muller, J.C. Oelrich, J. Paschopoulos, K.E. Rehm and H. Rosler, Jahresbericht 1977, Beschleunigerlaboratorium, Munchen (unpublished).

[121] J.D. Garrett, H.E. Wegner, T.M. Cormier, E.R. Cosman, O. Hansen and A.J. Lazzarini, Phys. Rev. C12 (1975) 489.

[122] M. Richter, W. Henning, H.J. Korner, R. Muller, K.E. Rehm, H.P. Rother, H. Schaller and H. Spieler, Nucl. Phys. A278 (1977) 163.

[123] H. Doubre, J.C. Jacmart, E. Plagnol, N. Poffé, M. Riou and J.C. Roynette, Phys. Rev. C15 (1977) 693.

[124] G.C. Li, I. Sick, R.R. Whitney and M.R. Yearian, Nucl. Phys. A162 (1971) 583.

[125] H. Bohn, K.A. Eberhard, R. Vandenbosch, K.G. Bernhardt, R. Bangert and Y-d. Chen, Phys. Rev. C16 (1977) 665.

[126] L.T. Chua, F.D. Becchetti, J. Janecke and F.L. Milder, Nucl. Phys. A273 (1976) 243.

[127] D. Scholz, H. Gemmeke, L. Lassen, R. Ost and K. Bethge, Nucl. Phys. A288 (1977) 351.

[128] R.M. DeVries, D.A. Goldberg, J.W. Watson, M.S. Zisman and J.G. Cramer, Phys. Rev. Lett. 39 (1977) 450.

[129] R.I. Cutler, M.J. Nadworny and K.W. Kemper, Phys. Rev. C15 (1977) 1318, and private communication.

[130] H.J. Gils, J. Buschmann, H. Rebel, G. Bechtold and S. Zagromski, Annual Report, Karlsruhe (1977).

[131] R.J. Puigh and K.W. Kemper, Nucl. Phys. A313 (1979) 363.

[132] R. Pardo, R.G. Markham, W. Benenson, A.E. Galonsky and E. Kashy, Phys. Lett. 71 B (1977) 301.

[133] G.R. Satchler, Phys. Lett. 83B (1979) 284.

[134] J.A. Jansen, R. Th. Peerdeman and C. DeVries, Nucl. Phys. A188 (1972) 337.

[135] R.J. Philpott, W.T. Pinkston and G.R. Satchler, Nucl. Phys. A119 (1968) 241.

[136] S. Cohen and D. Kurath, Nucl. Phys. A101 (1967) 1.

[137] R. Balzer, M. Hugi, B. Kamys, J. Lang, R. Muller, E. Ungricht, J. Unternahrer, L. Jarczyk and A. Strzalkowski, Nucl. Phys. A293 (1977) 518; E. Ungricht, private communication.

[138] D.A. Goldberg et al., unpublished; M.S. Zisman, private communication.

[139] D.P. Stahel, G.J. Wozniak, M.S. Zisman, B.D. Jeltema and J. Cerny, Phys. Rev. C16 (1977) 1456.

[140] G.R. Satchler, J.L.C. Ford, K.S. Toth, D.C. Hensley, E.E. Gross, D.E. Gustafson and S.T. Thornton, Phys. Lett. 60B (1975) 43.

[141] M.B. Johnson, L.W. Owen and G.R. Satchler, Phys. Rev. 142 (1966) 748.

[142] V.R.W. Edwards and B.C. Sinha, Phys. Lett. 37B (1971) 225; G.H. Rawitscher and R.A. Spicuzza, Phys. Lett. 37B (1971) 221.

[143] P.J. Moffa, J.P. Vary, C.B. Dover, C.W. Towsley, R.G. Hanus and K. Nagatani, Phys. Rev. Lett. 35 (1975) 992.

[144] D.A. Saloner and C. Toepffer, Nucl. Phys. A283 (1977) 108; D.A. Saloner, C. Toepffer and B. Fink, Nucl. Phys. A283 (1977) 131.

[145] G.R. Satchler, Phys. Lett. 33B (1970) 385.

[146] G.R. Satchler, Nucl. Phys. 55 (1964) 1.

[147] D.L. Hillis, E.E. Gross, D.C. Hensley, L.D. Rickertsen, C.R. Bingham, A. Scott and F.T. Baker, Phys. Rev. Lett. 36 (1976) 304. 
[148] H. Uberall, Electron scattering from complex nuclei (Academic Press, New York, 1971).

[149] L.W. Owen and G.R. Satchler, Nucl. Phys. 51 (1964) 155.

[150] R.P. Singhal, S.W. Brain, W.A. Gillespie, A. Johnston, E.W. Lees and A.G. Slight, Nucl. Phys. A218 (1974) 189.

[151] G.R. Satchler, J. Math. Phys. 13 (1972) 1118.

[152] S.J. Skorka, J. Hertel and T.W. Retz-Schmidt, Nucl. Data Tables A2 (1966) 347.

[153] S.W. Brain, A. Johnston, W.A. Gillespie, E.W. Lees and R.P. Singhal, J. Phys. G3 (1977) 821.

[154] P.H. Stelson and L. Grodzins, Nucl. Data 1A (1965) 1.

[155] F. Ajzenberg-Selove, Nucl. Phys. A248 (1976) 1.

[156] R.M. Haybron, M.B. Johnson and R.J. Metzger, Phys. Rev. 156 (1967).

[157] W.G. Love, A. Scott, F.T. Baker, W.P. Jones and J.D. Wiggins, Phys. Lett. 73B (1978) 277.

[158] W.G. Love, Nucl. Phys. A226 (1974) 319.

[159] F. Petrovich, Nucl. Phys. A251 (1975) 143;

F. Petrovich and D. Stanley, Nucl. Phys. A275 (1977) 487.

[160] D.M. Brink and G.R. Satchler, Angular Momentum (Oxford University Press, Oxford, 1971).

[161] A.K. Jain and C.S. Shastry, Phys. Rev. C19 (1979) 848.

[162] G.R. Satchler, submitted to Nucl. Phys. 